\title{
Multi-Watershed Nonpoint Source Pollution Management Through Coupling Bayesian-Based Simulation And Mechanism-Based Effluent Trading Optimization
}

\author{
Xiaomei Xu \\ Qingdao University \\ Xueting Zeng \\ Capital University of Economics and Business \\ Yongping Li \\ Beijing Normal University \\ Chunrong Wang \\ Qingdao University \\ Lei Yu \\ Zhengzhou University \\ Guohe Huang \\ University of Regina \\ Junlong Zhang ( $\square$ zhangjunlong@126.com ) \\ Qingdao University \\ Juan Feng \\ Qingdao University \\ Xinyu Han \\ Qingdao University
}

\section{Research Article}

Keywords: Bayesian inference, Constraint-violation risk, Multi-watershed management, Nonpoint source, Trading mechanism

Posted Date: June 3rd, 2021

DOI: https://doi.org/10.21203/rs.3.rs-503737/v1

License: (c) (1) This work is licensed under a Creative Commons Attribution 4.0 International License.

Read Full License 
Version of Record: A version of this preprint was published at Stochastic Environmental Research and Risk Assessment on November 17th, 2021. See the published version at https://doi.org/10.1007/s00477021-02130-w. 
Multi-watershed nonpoint source pollution management through coupling Bayesian-based simulation and mechanism-based effluent trading optimization

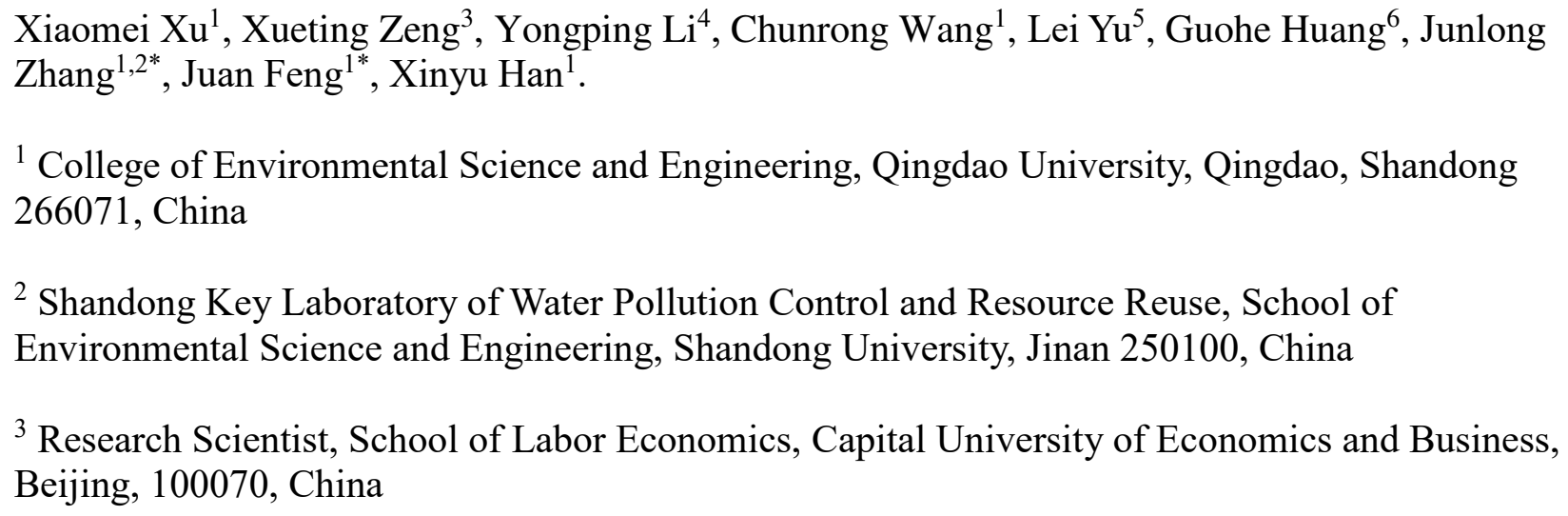




\section{Graphical Abstract}

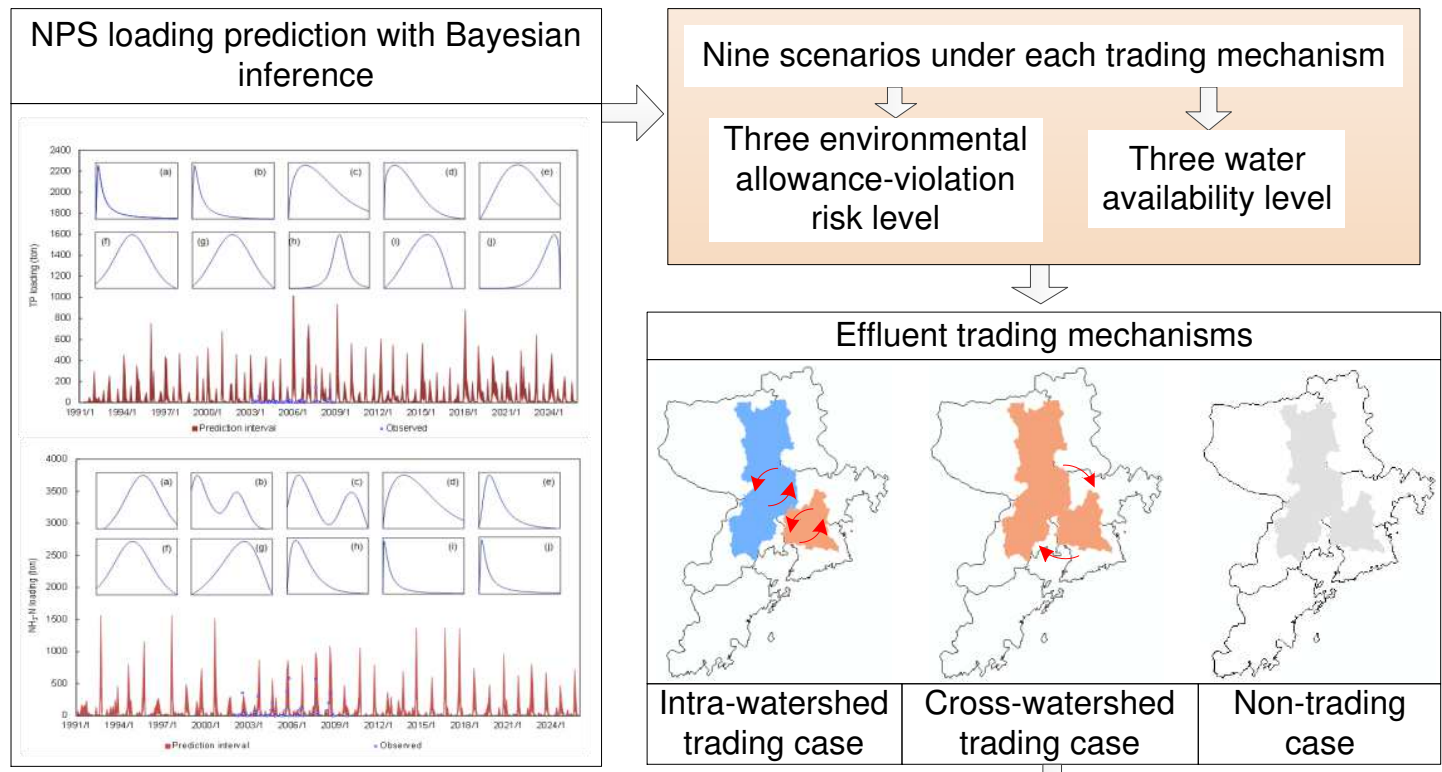

40

Which trading mechanism is recommended under different water availability level?

41 


\section{Highlights}

43

44 Bayesian inference provides random NPS loading prediction for optimization process.

45 The effects of allowance-violation risk and water availability level are analyzed.

46 Non-trading case generates highest benefits under low water availability level.

47 Cross-watershed trading performs best at medium and high water availability level.

48 The trading scale under cross-watershed trading would be highest. 


\section{Abstract}

51

52 Multiple rivers flowing into the same bay can be correlated in water quality management and together determine the environmental status of the bay. Nonpoint source pollution management for multi-watershed aiming to alleviate environmental contamination can be under additional challenges and yield considerable economic and environmental benefits. In this study, a Bayesian simulation-based multi-watershed effluent trading designing model (BS-METM) is established

57 for multi-watershed nonpoint source pollution management through incorporating techniques of water quality simulation, uncertainty analysis with Bayesian inference, optimal design for effluent trading, as well as mechanism analysis. BS-METM is capable of reflecting parameter uncertainties in nutrient simulation, disclosing the detailed optimal trading schemes under the impact of uncertainties and vital factors, and identifying optimal effluent trading mechanisms through revealing interaction among trading processes of multiple watersheds. BS-METM is applied to a real case of adjacent coastal watersheds (i.e. Daguhe and Moshuihe watersheds), which are identified as major sources of total phosphorus and ammonia nitrogen loadings to Jiaozhou Bay, China. Effluent trading optimization under multiple mechanisms, including intra-watershed trading, cross-watershed trading and non-trading, are conducted. The optimized industry scales and trading processes are obtained. The effects of vital factors on the trading process (i.e. environmental allowance-violation risk level and water availability level) are investigated. The interactions between water availability level and trading mechanism are also analyzed. It is proved that non-trading mechanism would be recommended under low water availability level and cross-watershed trading mechanism would be recommended under medium 
73 pollution management as well as effective sustainable development for multi-watershed region.

74

75 Keywords: Bayesian inference; Constraint-violation risk; Multi-watershed management;

76 Nonpoint source; Trading mechanism

77 


\section{Introduction}

The prevalence of nonpoint source (NPS) pollution, such as pollution generated by agricultural activities, has dramatically accelerated water quality deterioration (Zhang et al., 2009). Multiple rivers flowing into the same bay can be correlated in water quality management and together determine the environmental status of the bay. NPS management for multi-watershed aiming to alleviate environmental contamination can be under additional challenges and yield considerable economic and environmental benefits (Sith et al., 2019; Alnahit et al., 2020).

Effluent trading program provides flexibility of discharge permits to nonpoint and point sources and achieves optimal configuration for discharge permits, which can be a promising water quality management measure for pollution control across watersheds (Zeng et al., 2016). A number of literatures have been dedicated to designing trading system and recognizing optimal environmental and political factors (Clark et al., 2008; Nguyen et al., 2013; Chen et al., 2016; Zolfagharipoor and Ahmadi, 2017). For example, Hung and Shaw (2005) designed a trading-ratio system (TRS) for discharge permits in controlling water pollution, utilizing the unidirectional flow property of water; the TRS can meet the predetermined environmental quality standards within minimum aggregate abatement costs. Zhang et al. (2019) developed a Bayesian risk-induced interval stochastic modeling framework to disclose the interactions of trading ratio and treatment rate on effluent trading under system risk. Corrales et al. (2017) applied an integrated hydrology-economic modeling framework for assessing the effectiveness of effluent trading across two watersheds in Lake Okeechobee; the two-watershed phosphorus credit trading effort achieved a lower cost compared with command-and-control methods. Cross watershed 
101

102

103

104

105

106

107

108

109

110

111

112

113

114

115

116

117

118

119

120

121

122

123

trading has the potential to facilitate the water quality management system by enabling permit transaction among pollution sources with surplus discharge permits in multiple watersheds. The multi-watershed trading policy has a favorable role in promoting the development of green economy under certain conditions (Wang and Pang, 2019). Nevertheless, there have been few studies assessing factors on success of multi-watershed effluent trading programs as well as identifying optimal trading mechanisms.

In practical effluent trading, the trading planning can be affected due to various uncertainties. They are derived from variability in nutrient loadings and fluctuation in economic coefficients. Many stochastic mathematical programming methods have been extensively studied for supporting optimization modeling in water quality management under uncertainty ( $\mathrm{Li}$ and Huang, 2006; Maeda et al., 2009; Xu and Qin, 2010; Miao et al., 2014; Liu et al., 2016; Pastori et al., 2017). Among them, two-stage stochastic programming (TSP) can tackle decision-making problems related to randomness, which can utilize probability event of environmental penalties in the second-stage to rectify initial (first-stage) decision (Zeng et al., 2015; Rong et al., 2017; Chen et al., 2019; Wang, 2020). Chance constrained programming (CCP) is a programming method in addressing random variables' uncertainties on the right-hand side of the optimal models; it is capable of obtaining trading decision making through providing the trade-off analysis between system benefits and risk at different risk levels (Zhang et al., 2019). On the other hand, optimal design of trading planning can also be restricted by errors in hydrology/water quality simulation associated with a variety of complicated physical processes and spatiotemporal heterogeneity (Alam and Dutta, 2012; Shang et al., 2012; Shen et al., 2015; Shrestha and Wang, 2020). The errors in watershed process simulation lead to the research of Markov chain Monte Carlo 
124 (MCMC) sampling method (Laloy et al., 2013; Rajabi et al., 2015; Vrugt and Beven, 2018; Wu et

al., 2020). For example, Zhai et al. (2020a) quantified the parameter uncertainties of the dynamic constitutive model accurately by using Bayesian theory with Differential Evolution Adaptive Metropolis algorithm (DREAM). MCMC provides an efficient way to draw samples of parameter values from complex, high-dimensional statistical distributions in a Bayesian framework. Thus an integration model framework with Bayesian inference based simulation efforts and optimization approaches is desired to be developed for improving the accuracy in nutrient fate modeling as well as to accounting for uncertainties in water quality management problems.

Therefore, this study aims to propose a Bayesian simulation-based multi-watershed effluent trading designing model (BS-METM) for multi-watershed water quality management. BS-METM combines uncertainty analysis of SWAT model with MCMC, constraint-violation risk-based two-stage stochastic programming (CRTSP) and mechanism analysis into a general framework. According to the water quality protocols based on SWAT model, Bayesian estimation (DREAM algorithm) is used to analyze the parameters' posterior distributions and the nutrient loadings' simulated uncertainty ranges for agriculture. The uncertainty ranges can provide random inputs of NPS loading in order to gain optimal effluent trading schemes. CRTSP couples TSP, CCP and interval parameter linear programming (ILP) optimization approaches, which is capable of disclosing optimal industry scales and trading process as well as identifying the best trading mechanism. The mechanism analysis can compare the trading schemes under intra-watershed trading, cross-watershed trading and non-trading cases based on the performance of net system benefits, excess nutrient emissions, trading amount of pollution permits and eliminated permits from trading market. The BS-METM will be applied in a real case of water 
147 quality management for two agricultural watersheds, Daguhe and Moshuihe watersheds, close to 148 Jiaozhou Bay, China. The modeling framework will (i) disclose the detailed optimized effluent 149 trading planning between every pair of pollutant sources under multiple uncertainties and system 150 risks; (ii) reveal the effects of vital factors on the trading process (i.e. environmental 151 allowance-violation risk level and water availability level); (iii) analyze effluent trading under 152 different trading mechanisms and select the best mechanism to make policy suggestions.

\section{Methodology}

155

\subsection{DREAM algorithm within Bayesian inference}

157

Dream is a multi-chain and self-adaptive differential evolutionary probability sampling method based on Bayesian theory (Vrugt JA, 2016). It generally does not depend on the prior distribution determined artificially. In addition, the offset abnormal chains can be removed (Zhai et al., 2020a). DREAM procedure steps are as follows (Vrugt JA, 2009; Sheng et al., 2019):

1) Each Markov chain's initial value is derived from determined the parametric prior distribution, denoted as $\phi^{i}(i=1,2, \ldots, N)$.

2) The initial value's likelihood of each chain can be calculated:

$$
\pi\left(\phi^{i}\right)=f\left(\phi^{i} \mid \delta\right)
$$

3) Candidate samples are generated by mutation operation. In addition, perform candidate samples are cross-operated according to the crossover probability $C R \in[0,1]$, namely the 
crossover probability. The likelihood and receptance rate of the newly gained candidate sample $Z_{j . t+1}$ are computed. If the receptance rate $\alpha\left(\phi_{j . t}, Z_{j . t+1}\right)>U$ (sampling from uniform distribution $U(0,1)$ ), otherwise refuse. The Inter-Quartile-Range (IQR) method can remove the useless chain.

4) Exit conditions on account of convergent judgment are calculated. If the convergence criterion is reached, the calculation will end. Or else, step (3) will always be repeated to develop the Markov chain. When the scale down factor of each parameter in DREAM algorithm: $R_{\text {stat }}<1.2$, the posterior distribution of the parameter is stably converged. The formula for $R_{\text {stat }}$ is:

$$
R_{\text {stat }}=\sqrt{\left(1-\frac{1}{J}\right)+\frac{N+1}{N W} \frac{B}{J}}
$$

where, $J$ is the sample number of each chain, $N$ is the number of Markov chains, $B / J$ is the variance of the mean value of $J$ Markov chains. $W$ is the mean value of the variance of $J$ Markov chains. DREAM algorithm is used for uncertainty analysis for parameters in SWAT model. Nutrient (e.g. $\mathrm{NH}_{3}-\mathrm{N}$ and TP) migration eqautions etc. based on SWAT are shown in Appendix A.

\subsection{Interval two-stage stochastic programming with constraint-violation risk}

In a decision problem with risk or penalty, two-stage stochastic programming method will be provided to deal with stochastic uncertainty of parameters ( $\mathrm{Li}$ et al., 2008). The basic idea is the 
207

208

209 concept of recourse, which is to take up remedial measures, reduce the environment penalties or curtail activity plans after the occurrence of a random event. In this problem, a first-stage decision of production targets are formulated before the random nutrient emission is achieved (Li and Huang, 2008). For example, the decision variables of the first stage can be the scales of livestock and poultry industry and fishery, production level of companies as well as targeted area of agriculture. In order to minimize the possible penalties owing to the infeasibility of the first-stage decision after a random event has occurred, the recourse action to correct the benefits of the first stage through the second-stage penalties would be taken. For example, the second-stage variables can be the excess annual $\mathrm{NH}_{3}-\mathrm{N}$ and TP loadings from the nonpoint sources. Although the stochastic uncertainty of nutrient loadings emission would be effectively reflected with the two-stage stochastic programming model, the uncertainties of other parameters couldn't be addressed with it. For example, the parameters on economy, energy and protein may not be used as definite values. So, interval parameters are introduced on the basis of TSP framework to reflect the uncertainty of this kind of parameters, transmitting the uncertain information in economic, energy and protein parameters to the optimization process, which forms an interval two-stage stochastic programming (ITSP) model (Huang, 2000; Zhang, 2019):

$$
\operatorname{Max} f^{ \pm}=\sum_{j=1}^{n_{1}} c_{j}^{ \pm} x_{j}^{ \pm}-\sum_{j=1}^{n_{2}} \sum_{h=1}^{v} p_{h} d_{j}^{ \pm} y_{j h}^{ \pm}
$$

Subject to:

$$
\sum_{j=1}^{n_{1}} a_{r j}^{ \pm} x_{j}^{ \pm} \leq b_{r}^{ \pm}, r=1,2, \ldots, m_{1}
$$

$$
\sum_{j=1}^{n_{1}} a_{t j}^{ \pm} x_{j}^{ \pm}+\sum_{j=1}^{n_{2}} a_{t j}^{ \pm \pm} y_{j h}^{ \pm} \geq w_{t}^{ \pm}, t=1,2, \ldots m_{2}
$$




$$
x_{j}^{ \pm} \geq 0, j=1,2, \ldots n_{1}
$$

$$
y_{j h}^{ \pm} \geq 0, j=1,2, \ldots n_{2} ; h=1,2, \ldots v
$$

where superscripts " -" and "+ " represent the lower and upper bounds of an interval parameter or variable, respectively; $j$ denotes the pollution sources; $t$ and $r$ are marks of constraints; $h$ represents the probability level; $f^{ \pm}, c_{j}^{ \pm}, d_{j}^{ \pm}, a_{r j}^{ \pm}, a_{t j}^{ \pm}, a_{t j}^{\prime \pm}, b_{r}^{ \pm}, x_{j}^{ \pm}, y_{j h}^{ \pm}$and $w_{t}^{ \pm}$are interval coefficients/objectives that are presented as interval numbers. For example, letting $b_{r}^{-}$ and $b_{r}^{+}$be lower and upper bounds of interval number $b_{r}^{ \pm}$, meanwhile, $b_{r}^{ \pm}=\left[b_{r}^{-}, b_{r}^{+}\right]$.

However, the right-hand side parameters (e.g. environmental capacity of $\mathrm{NH}_{3}-\mathrm{N}$ in Daguhe watershed) involve randomness originating from spatiotemporal heterogeneity in practical effluent trading. The above problem cannot be addressed with the interval two-stage stochastic programming model. While, Chance-constrained programming (CCP) is introduced to deal with the random uncertainties and analyze constraint-violation risk effectively (Zhu et al., 2012; Piao et al., 2014), as follows:

$$
\operatorname{Max} f=C(t) X
$$

226 Subject to:

$$
\operatorname{Pr}\left[\left\{t \mid A_{u}(t) X \geq b_{u}(t)\right\}\right] \geq \alpha_{u}
$$

$$
A_{u}(t) \in A(t), b_{u}(t) \in B(t), u=1,2, \ldots \ldots ., U
$$

$X \geq 0$ 
232 where $X$ denotes a vector of decision variables; $A(t), B(t)$, and $C(t)$ are sets with 233 random elements defined on a probability space $T, t \in T$; model (4b) consists of a prescribed 234 level of probability $\alpha_{u} \in[0,1]$ for each constraint $u$ and imposes a condition that the 235 constraint is satisfied with at least a probability of $1-\alpha_{u}$ (Xie et al., 2011). When $A(t)$ are 236 deterministic and $b_{u}(t)$ are stochastic, constraint (4b) becomes linear:

$$
A_{u} X \geq b_{u}(t)^{1-\alpha_{u}}
$$

Equation (5) is equivalent to equation (4b), given the cumulative distribution function of $b_{u}$ and the probability of violating constraint $u$. Accordingly, CCP and ITSP can be integrated to deal with multiple uncertainties existing in the objective function and constraints, which leads to a two-stage stochastic programming with constraint-violation risk (TSPCR) model as follows:

$$
\operatorname{Max} f^{ \pm}=\sum_{j=1}^{n_{1}} c_{j}^{ \pm} x_{j}^{ \pm}-\sum_{j=1}^{n_{2}} \sum_{h=1}^{v} p_{h} d_{j}^{ \pm} y_{j h}^{ \pm}
$$

244 Subject to:

$$
\sum_{j=1}^{n_{1}} a_{r j}^{ \pm} x_{j}^{ \pm} \leq b_{r}^{ \pm}, r=1,2, \ldots, m_{1}
$$

$$
\sum_{j=1}^{n_{1}} a_{t j}^{ \pm} x_{j}^{ \pm}+\sum_{j=1}^{n_{2}} a_{t j}^{ \pm \pm} y_{j h}^{ \pm} \geq\left(w_{t}^{ \pm}\right)^{1-\alpha_{u}}, t=1,2, \ldots m_{2}
$$

$$
x_{j}^{ \pm} \geq 0, j=1,2, \ldots n_{1}
$$


250 In above model, $\left(w_{t}^{ \pm}\right)^{1-\alpha_{u}}$ is the stochastic coefficient under period $\mathrm{t}$ and under

251 constraint-violation probability $\alpha_{u}$. Model (6) can be transformed into two sub-models which

252 correspond to lower and upper bounds of the target function values (Huang et al., 2000). Then the 253 interval solutions can be gained by solving two sub-models in sequence.

\section{Case Study}

\subsection{Study area}

Daguhe watershed is the largest river of all the rivers flowing into Jiaozhou Bay, with a length of more than $140 \mathrm{~km}$. And the total area is $4631 \mathrm{~km}^{2}$, which is located between latitudes of $36^{\circ} 10^{\prime} \mathrm{N}-37^{\circ} 12^{\prime} \mathrm{N}$ and longtitudes of $120^{\circ} 03^{\prime} \mathrm{E}-120^{\circ} 25^{\prime} \mathrm{E}$ in the northwestern part of Qingdao, China (Chen et al., 2010). It flows through Laixi, Pingdu, Jiaozhou and Jimo cities, accounting for $45 \%$ of the total area of Qingdao. The region has an average annual precipitation of $707.4 \mathrm{~mm}$ and an average annual temperature of $10-11^{\circ} \mathrm{C}$ with a warm temperate coastal humid monsoon climate in North China. Brown soil, tidal soil and sandy ginger and black soil are the main soil types within the watershed (Liao et al, 2010; Sun et al, 2016). Moshuihe watershed has a length of $21.3 \mathrm{~km}$ and a total area of $276.1 \mathrm{~km}^{2}$ with an average annual precipitation of $680 \mathrm{~mm}$ (Qiao et al., 2012). Moshuihe watershed flows through Jimo city into Jiaozhou Bay, which includes four main tributaries including Liucun River, Longquan River, Tuqiaotou River and Xifengliu River. 
270 The main agricultural crops for the two watersheds are wheat, corn and peanut, chinese cabbage, 271 celery, carrot, potato, apple, pear, peach and grape and the main livestocks are chicken, pig, cattle 272 and cow.

On one hand, Daguhe and Moshuihe watersheds are agricultural watersheds. To meet the increasing food demand, long-term utilization of fertilizers and manures make agricultural nitrogen and phosphorus pollution be in a high status. Intensive livestock and poultry industry and fishery can also be factors that trigger NPS pollution, which can be regarded as a major threat to water quality of the two watersheds and Jiaozhou Bay. $\mathrm{NH}_{3}-\mathrm{N}$ and $\mathrm{TP}$ are two main contaminants for the watersheds. The amount of TP flowing into Jiaozhou Bay through Daguhe watershed accounts for $55.34 \%$ of total TP loading to the Bay. The amount of $\mathrm{NH}_{3}-\mathrm{N}$ accounts for $24.18 \%$ of total $\mathrm{NH}_{3}-\mathrm{N}$ loading to Jiaozhou Bay (Li et al., 2009). On the other hand, Daguhe watershed and Moshuihe watershed are two adjacent rivers flowing into Jiaozhou Bay and correlated in water quality management. They together determine the environmental status of Jiaozhou Bay. Thus, it is desired that an effective system analysis method be advanced to accomplish a sound decision scheme for multi-watershed NPS pollution control. Figure 1 shows the general framework of the advocated BS-METM. The system incorporates uncertainty analysis of SWAT model with MCMC, constraint-violation risk-based two-stage stochastic programming (CRTSP) and mechanism analysis. Each part has a distinctive contribution as shown in introduction to improve the capability of the model in dealing with complexities in effluent trading planning. 


\subsection{Modeling formulation}

The ecological environment of Daguhe and Moshuihe watersheds is extremely vulnerable because of the excessive nutrient emission. The improper allocation of discharge permits strategy may lead to the inefficiency of environmental management even lead to the issue of hot spots. Instead, effluent trading would contribute to allocate discharge permits with optimal economic benefits or enhanced environmental benefits. In addition, those with pollution sources that are not easy to mitigate or who choose to heighten production can purchase the unused permits deriving from the others within the trading system, without paying huge environmental penalties. TP and $\mathrm{NH}_{3}-\mathrm{N}$ are selected as water quality indicators.

As shown in Figure 2, firstly, six reaches have been demarcated in the two watersheds for avoiding the issue of hot spots in trading, including 4 reaches in Daguhe watershed and 2 reaches in Moshuihe watershed (Xu, 2004; Ning et al., 2017). Secondly, there are 18 major pollution sources in the two watersheds, including 10 nonpoint sources in Daguhe watershed (i.e. four agricultural zones, three livestock and poultry industry zones and three fishery zones) as well as one agricultural zone in Moshuihe watershed. Besides, seven companies in Moshuihe watershed are also considered. The planning period in this study is one year (2021), and the discharge permit trading of three levels of water availability (high level $(w=1)$, low level $(w=2)$ and medium level $(w=3))$ is respectively planned. In study area, $\mathrm{TP}$ and $\mathrm{NH}_{3}-\mathrm{N}$ discharge permits would be allocated to 18 pollution sources in two watersheds, which include multiple human 
activities (i.e. agriculture, livestock and poultry industry, fishery and company). The initial

317 allocation is based on the proportion of their own ecological, economic benefits and pollutant

318 emissions. BS-METM can be formulated under three trading mechanism cases. Under Case 1, the

319 discharge permits are forced to be traded only within the pollution sources from the same

320 watershed. Under Case 2, cross-watershed effluent trading is allowed, which means that the pollution sources in Daguhe watershed can be traded with those in Moshuihe watershed. Under

Case 3, cross-watershed environmental constraints are allowed but all pollution sources are not traded for discharge permits. The models of effluent trading under Case 1 and 3 are provided in

Compressed File. The model of effluent trading scheme under Case 2 can be formulated as follows:

$$
\begin{aligned}
\operatorname{Maxf}= & \sum_{i=1}^{5} \sum_{j=1}^{11} \sum_{w=1}^{3} A B_{j}^{ \pm} \cdot\left(X_{i j}^{-}+\Delta X_{i j} \cdot o_{i j w}\right)+\sum_{\mathrm{n}=1}^{3} \sum_{\mathrm{r}=1}^{4} \sum_{w=1}^{3} W_{r}^{ \pm} \cdot\left(N_{n r}^{-}+\Delta N_{n r} \cdot r_{n r w}\right) \\
& +\sum_{p=1}^{3} \sum_{w=1}^{3} S B_{p}^{ \pm} \cdot\left(Z_{p}^{-}+\Delta Z_{p} \cdot S_{p w}\right)+\sum_{m=1}^{7} \sum_{w=1}^{3} C B_{m}^{ \pm} \cdot\left(Y_{m}^{-}+\Delta Y_{m} \cdot e_{m w}\right) \\
& -\sum_{i=1}^{5} \sum_{k=1}^{3} \sum_{w=1}^{3} h_{k} \cdot E D P A_{i k w}^{ \pm} \cdot P F^{ \pm}-\sum_{n=1}^{3} \sum_{w=1}^{3} E D P R_{n w}^{ \pm} \cdot P F^{ \pm} \\
& -\sum_{p=1}^{3} \sum_{w=1}^{3} E D P P_{p w}^{ \pm} \cdot P F^{ \pm}-\sum_{m=1}^{7} \sum_{w=1}^{3} E D P C_{m w}^{ \pm} \cdot P F^{ \pm} \\
& -\sum_{i=1}^{5} \sum_{s=1}^{3} \sum_{w=1}^{3} k_{s} \cdot E D N A_{i s w}^{ \pm} \cdot N F^{ \pm}-\sum_{n=1}^{3} \sum_{w=1}^{3} E D N R_{n w}^{ \pm} \cdot N F^{ \pm} \\
& -\sum_{p=1}^{3} \sum_{w=1}^{3} E D N P_{p w}^{ \pm} \cdot N F^{ \pm}-\sum_{m=1}^{7} \sum_{w=1}^{3} E D N C_{m w}^{ \pm} \cdot N F^{ \pm}
\end{aligned}
$$


332 The objective is to maximize the ultimate net system benefit, which is calculated with the total 333 environmental penalty and the total initial net system benefit which removes the cost. The

334 ultimate system net benefit considers the total initial net system benefits and the total

335 environmental penalties of agriculture, livestock and poultry industry, fishery and company. The 336 constraints to be complied with can be divided into the following groups:

337

1. Constraints for TP permit reallocation

340

$$
\sum_{j=1}^{11}\left(X_{i j}^{-}+\Delta X_{i j} \cdot o_{i j w}\right) \cdot C W P A_{k}^{ \pm}-E D P A_{i k w}^{ \pm} \leq A C E P_{i w}
$$

$$
\sum_{r=1}^{4}\left(N_{n r}^{-}+\Delta N_{n r} \cdot r_{n r w}\right) \cdot C W P R_{r}^{ \pm}-E D P R_{n w}^{ \pm} \leq L C E P_{n w}
$$

$$
\left(Z_{p}^{-}+\Delta Z_{p} \cdot s_{p w}\right) \cdot D W P^{ \pm} \cdot C W P P^{ \pm}-E D P P_{p w}^{ \pm} \leq S C E P_{p w}
$$

$$
\left(Y_{m}^{-}+\Delta Y_{m} \cdot e_{m w}\right) \cdot D W M_{m}^{ \pm} \cdot C W P M_{m}^{ \pm}-E D P C_{m w}^{ \pm} \leq C C E P_{m w}
$$


350 Constraints $(7 \mathrm{j})-(7 \mathrm{~m})$ and $(7 \mathrm{n})-(7 \mathrm{q})$ represent the trading process. The discharge permits are

351 traded among 18 sources of pollution, including cross-watershed agricultural zones, livestock and

352 poultry industry zones, fishery zones and companies. In addition, the reallocated $\mathrm{TP}$ and $\mathrm{NH}_{3}-\mathrm{N}$

353 discharge permits for each pollution source in Daguhe and Moshuihe watersheds are equal to that

354 the initial discharge permits plus the purchasing permits, and minus the selling permits.

355

356 3. Constraints for TP trading rules

357

$$
\begin{aligned}
& \sum_{i^{\prime}=1}^{5} T P_{i i^{\prime} w}+\sum_{n=1}^{3} T P s_{i n w}+\sum_{p=1}^{3} T P s_{i p w}+\sum_{m=1}^{7} T P s_{i m w} \\
& \leq T P I_{i w}+\sum_{i^{\prime}=1}^{5} T P_{i^{\prime} i w} / t p_{i^{\prime} i}+\sum_{m=1}^{7} T P b_{m i w} / t p_{m i}+\sum_{n=1}^{3} T P b_{n i w} / t p_{n i}+\sum_{p=1}^{3} T P b_{p i w} / t p_{p i} \\
& \sum_{n^{\prime}=1}^{3} T P_{n n^{\prime} w}+\sum_{i=1}^{5} T P s_{n i w}+\sum_{p=1}^{3} T P s_{n p w}+\sum_{m=1}^{7} T P s_{n m w} \\
& \leq T P N_{n w}+\sum_{n^{\prime}=1}^{3} T P_{n^{\prime} n w} / t p_{n^{\prime} n}+\sum_{i=1}^{5} T P b_{i n w} / t p_{i n}+\sum_{p=1}^{3} T P b_{p n w} / t p_{p n}+\sum_{m=1}^{7} T P b_{m n w} / t p_{m n}
\end{aligned}
$$

$\sum_{p^{\prime}=1}^{3} T P_{p p^{\prime} w}+\sum_{i=1}^{5} T P s_{p i w}+\sum_{n=1}^{3} T P s_{p n w}+\sum_{m=1}^{7} T P s_{p m w}$

$$
\leq T P P_{p w}+\sum_{p^{\prime}=1}^{3} T P_{p^{\prime} p w} / t p_{p^{\prime} p}+\sum_{n=1}^{3} T P b_{n p w} / t p_{n p}+\sum_{i=1}^{5} T P b_{i p w} / t p_{i p}+\sum_{m=1}^{7} T P b_{m p w} / t p_{m p}
$$

360

$$
\begin{aligned}
& \sum_{m^{\prime}=1}^{7} T P_{m m^{\prime} w}+\sum_{i=1}^{5} T P s_{m i w}+\sum_{n=1}^{3} T P s_{m n w}+\sum_{p=1}^{3} T P s_{m p w} \\
& \leq T P M_{m w}+\sum_{m^{\prime}=1}^{7} T P_{m^{\prime} m w} / t p_{m^{\prime} m}+\sum_{i=1}^{5} T P b_{i m w} / t p_{i m}+\sum_{n=1}^{3} T P b_{n m w} / t p_{n m}+\sum_{p=1}^{3} T P b_{p m w} / t p_{p m}
\end{aligned}
$$

4. Constraints for $\mathrm{NH}_{3}-\mathrm{N}$ trading rules 


$$
\begin{aligned}
& \sum_{i^{\prime}=1}^{5} T N_{i i^{\prime} w}+\sum_{n=1}^{3} T N s_{i n w}+\sum_{p=1}^{3} T N s_{i p w}+\sum_{m=1}^{7} T N s_{i m w} \\
& \leq T N I_{i w}+\sum_{i^{\prime}=1}^{5} T N_{i^{\prime} i w} / t n_{i^{\prime} i}+\sum_{m=1}^{7} T N b_{m i w} / t n_{m i}+\sum_{n=1}^{3} T N b_{n i w} / t n_{n i}+\sum_{p=1}^{3} T N b_{p i w} / t n_{p i}
\end{aligned}
$$

$$
\begin{aligned}
& \sum_{n^{\prime}=1}^{3} T N_{n n^{\prime} w}+\sum_{i=1}^{5} T N s_{n i w}+\sum_{p=1}^{3} T N s_{n p w}+\sum_{m=1}^{7} T N s_{n m w} \\
& \leq T N N_{n w}+\sum_{n^{\prime}=1}^{3} T N_{n^{\prime} n w} / t n_{n^{\prime} n}+\sum_{i=1}^{5} T N b_{i n w} / t n_{i n}+\sum_{p=1}^{3} T N b_{p n w} / t n_{p n}+\sum_{m=1}^{7} T N b_{m n w} / t n_{m n}
\end{aligned}
$$

365

$$
\sum_{p^{\prime}=1}^{3} T N_{p p^{\prime} w}+\sum_{i=1}^{5} T N s_{p i w}+\sum_{n=1}^{3} T N s_{p n w}+\sum_{m=1}^{7} T N s_{p m w}
$$

$$
\leq T N P_{p w}+\sum_{p^{\prime}=1}^{3} T N_{p^{\prime} p w} / t n_{p^{\prime} p}+\sum_{n=1}^{3} T N b_{n p w} / t n_{n p}+\sum_{i=1}^{5} T N b_{i p w} / t n_{i p}+\sum_{m=1}^{7} T N b_{m p w} / t n_{m p}
$$

366

$$
\begin{aligned}
& \sum_{m^{\prime}=1}^{7} T N_{m m^{\prime} w}+\sum_{i=1}^{5} T N s_{m i w}+\sum_{n=1}^{3} T N s_{m n w}+\sum_{p=1}^{3} T N s_{m p w} \\
& \leq T N M_{m w}+\sum_{m^{\prime}=1}^{7} T N_{m^{\prime} m w} / t n_{m^{\prime} m}+\sum_{i=1}^{5} T N b_{i m w} / t n_{i m}+\sum_{n=1}^{3} T N b_{n m w} / t n_{n m}+\sum_{p=1}^{3} T N b_{p m w} / t n_{p m}
\end{aligned}
$$

Constraints $(7 \mathrm{j})-(7 \mathrm{~m})$ and $(7 \mathrm{n})-(7 \mathrm{q})$ can contribute to ensure that the selling TP and $\mathrm{NH}_{3}-\mathrm{N}$ discharge permits from pollution sources should be larger than the initial permits they possess, respectively.

$$
A C E P_{i w} \leq T P A_{i w}, \quad L C E P_{n w} \leq T P L_{n w}
$$

$$
S C E P_{p w} \leq T P S_{p w}, \quad C C E P_{m w} \leq T P C_{m w}
$$

$$
A C E P_{i w}+L C E P_{n w}+S C E P_{p w} \leq T P G F_{q w} \quad \forall i=n=p, i=1,2,3, q \neq 4
$$




$$
\begin{array}{ll}
376 & A C E P_{i w} \leq T P G F_{q w} \quad i=4, q=4 \\
377 & A C E P_{5 w}+C C E P_{3 w}+C C E P_{4 w}+C C E P_{5 w}+C C E P_{6 w} \\
378 & C C E P_{1 w}+C C E P_{2 w}+C C E P_{7 w} \leq T P W F_{2 w} \\
379 \quad & \sum_{i=1}^{4} A C E P_{i w}+\sum_{n=1}^{3} L C E P_{n w}+\sum_{p=1}^{3} S C E P_{p w} \leq T P G_{w}{ }^{1-p_{h}} \\
380 & A C E P_{i w}+\sum_{m=1}^{7} C C E P_{m w} \leq T P W_{w} i=5 \\
& \sum_{i=1}^{5} A C E P_{i w}+\sum_{n=1}^{3} L C E P_{n w}+\sum_{p=1}^{3} S C E P_{p w}+\sum_{m=1}^{7} M C E P_{m w} \leq T P T_{w}
\end{array}
$$

\section{2}

6. Constraints for $\mathrm{NH}_{3}-\mathrm{N}$ environmental limit

$$
\begin{aligned}
& A C E N_{i w} \leq T N A_{i w}, \quad \operatorname{LCEN}_{n w} \leq T N L_{n w} \\
& \operatorname{SCEN}_{p w} \leq T N S_{p w}, \quad C C E N_{m w} \leq T N C_{m w} \\
& A C E N_{i w}+\operatorname{LCEN}_{n w}+\operatorname{SCEN}_{p w} \leq T N G F_{q w} \quad \forall i=n=p, i=1,2,3, q \neq 4
\end{aligned}
$$$$
A C E N_{i w} \leq T N G F_{q w} \quad i=4, q=4
$$$$
A C E N_{5 w}+C C E N_{3 w}+C C E N_{4 w}+C C E N_{5 w}+C C E N_{6 w} \leq T N W F_{1 w}
$$$$
390 \quad \sum_{i=1}^{4} A C E N_{i w}+\sum_{n=1}^{3} \operatorname{LCEN}_{n w}+\sum_{p=1}^{3} \operatorname{SCEN}_{p w} \leq \operatorname{TNG}_{w}{ }^{1-p_{h}}
$$

$$
A C E N_{i w}+\sum_{m=1}^{7} \operatorname{CCEN}_{m w} \leq \operatorname{TNMM}_{w} \quad i=5
$$




$$
\sum_{i=1}^{5} A C E N_{i w}+\sum_{n=1}^{3} \operatorname{LCEN}_{n w}+\sum_{p=1}^{3} \operatorname{SCEN}_{p w}+\sum_{m=1}^{7} \operatorname{CCEN}_{m w} \leq T N T_{w}
$$

The environmental restrictions for $\mathrm{TP}$ and $\mathrm{NH}_{3}-\mathrm{N}$ are set for the four industries, the four reaches in Daguhe watershed, the two reaches in Moshuihe watershed, the whole Daguhe watershed, the whole Moshuihe watershed and the cross watersheds in constraints (7r)-(7y) and (7z)-(7ah).

7. Energy and protein requirements constraints for cross-watersheds

400

$$
\begin{aligned}
& \sum_{i=1}^{5} \sum_{j=1}^{11} A P_{i j}^{ \pm} \cdot\left(X_{i j}^{-}+\Delta X_{i j} \cdot o_{i j w}\right) \cdot P E_{j}-\sum_{n=1}^{3} \sum_{r=1}^{4} L E_{r} \cdot\left(N_{n r}^{-}+\Delta N_{n r} \cdot r_{n r w}\right) \\
& -D P \cdot N E-M P \cdot N E \geq 0 \\
& \sum_{i=1}^{5} \sum_{j=1}^{11} A P_{i j}^{ \pm} \cdot\left(X_{i j}^{-}+\Delta X_{i j} \cdot o_{i j w}\right) \cdot C E_{j}-\sum_{n=1}^{3} \sum_{r=1}^{4} L P_{r} \cdot\left(N_{n r}^{-}+\Delta N_{n r} \cdot r_{n r w}\right) \\
& -D P \cdot N P-M P \cdot N P \geq 0
\end{aligned}
$$

401

402

403

Constraint (7ai) and (7aj) represent that the energy and digestible protein content in cross-watersheds crops should be larger than the demands of humans and livestocks, respectively.

404

405

406

8. Technical and non-negativity constraints

$$
0 \leq o_{i j w} \leq 1,0 \leq r_{n r w} \leq 1,0 \leq s_{p w} \leq 1,0 \leq e_{m w} \leq 1
$$

407

Besides, the technology and non-negative constraints comprise other decision variables in the 409 model, including the excess $\mathrm{TP}$ and $\mathrm{NH}_{3}-\mathrm{N}$ emission of each pollution source and the trading 410 amount between two sources. In addition, the excess TP and $\mathrm{NH}_{3}-\mathrm{N}$ emission of each pollution 
411

412

source are lower than the total $\mathrm{TP}$ and $\mathrm{NH}_{3}-\mathrm{N}$ emission from the source, respectively; the sources' reallocated TP and $\mathrm{NH}_{3}-\mathrm{N}$ emission permits should be higher than the minimum reallocated emission permits, which are set as $25 \%$ of the initial emission permits in this study; the TP and $\mathrm{NH}_{3}-\mathrm{N}$ emission permits sold from source A to source B should be equal to the emission permits purchased from source A by source B, such as $T P b_{\text {niw }}=T P s_{\text {niw }}$, and the TP and $\mathrm{NH}_{3}-\mathrm{N}$ trading amount should be lower than the initial pollutant discharge permit of the source. Nomenclature of the model is as shown in Appendix B.

\subsection{Data collection}

In this study, a range of general meteorological data associated with model inputs are demanded for watershed hydrological simulation, including maximum and minimum temperature and daily rainfall data during the period of 1991-2018 (China Meteorological Data Service Center:

CMDC). Using digital elevation model (DEM), the physical characteristics, flow direction and hydrological network of rivers are calculated. DEM data set is gained from the Geospatial Data Cloud site, Computer Network Information Center, Chinese Academy of Sciences (http://www.gscloud.cn), with a resolution of $90 \mathrm{~m}$. The 2000, 2010 and 2018 land use datasets were provided by Data Center for Resources and Environmental Sciences, Chinese Academy of Sciences (RESDC) (http://www.resdc.cn). The 1:1 million soil map was collected from Soil and Terrain (SOTER) database (Zhang and Zhao, 2008). The relevant uncertain data of each pollution source, represented by interval values due to the uncertainty of the obtained information were derived from historical records (e.g. Qingdao statistical yearbook) and by field survey. The 
433 discharge permits initially allocated to each industry are based on nutrient loadings, net economic

434 benefits and net ecological benefits. The analytic hierarchy process (AHP) was used to determine 435 three factors concerning weights.

\section{Results and Discussion}

\subsection{Prediction of nutrient loadings with SWAT}

441 In this study, parameters' uncertainties in modeling the fate of $\mathrm{TP}$ and $\mathrm{NH}_{3}-\mathrm{N}$ from nonpoint sources were assessed; the associated nutrient loadings with random characteristics were predicted. The results would be used as the random inputs of optimization process to gain the sound effluent trading schemes. Twenty most sensitive parameters for $\mathrm{TP}$ and $\mathrm{NH}_{3}-\mathrm{N}$ in modeling nutrient loadings were identified with LH-OAT (Latin Hypercube One-factor-at-a-time) technique as shown in Table 1. The parameter uncertainties for the sensitive parameters and the related nutrient fate simulation were addressed by using MCMC method with DREAM (Differential Evolution Adaptive Metropoils) algorithm. Parameters were estimated with eight Markov chains comprising 20,000 iterations for each parameter, and the first $10 \%$ of which would be removed as burn in period. Their prior densities were designed to be uniform within their limits as shown in Table 1.

452

Place Table 1 here

454 
456 The marginal posterior probability density distributions for the parameters of $\mathrm{TP}$ and $\mathrm{NH}_{3}-\mathrm{N}$ are

457 highlighted as shown in Figures 3 and 4. The prediction intervals of TP and $\mathrm{NH}_{3}-\mathrm{N}$ loadings are

458 acquired by running SWAT model for years 1991-2025 (Figures 3 and 4). The parameter

459 uncertainties are analyzed based on the observed data for $\mathrm{TP}$ and $\mathrm{NH}_{3}-\mathrm{N}$ loadings, which are

460 highlighted with blue circles. The results depict that $31.3 \%$ and $51.3 \%$ of the observed TP and

$461 \mathrm{NH}_{3}-\mathrm{N}$ loadings are captured by the uncertain range, respectively. The simulated lower and upper

462 time series of $\mathrm{TP}$ and $\mathrm{NH}_{3}-\mathrm{N}$ loading are statistically analyzed in order to obtain random inputs

463 for the effluent trading planning. Figure 5 shows the cumulative probability distribution function

464 (CDFs) under the three generation levels and lower- and upper-bound TP loading. The best

465 cumulative probability distributions for the upper- and lower-bound time series of TP loading at

466 any emission level are Rician and Rayleigh distributions, respectively. Figure 6 displays the

467 cumulative probability distribution function (CDFs) under the three generation levels of lower-

468 and upper-bound $\mathrm{NH}_{3}-\mathrm{N}$ loading. The best cumulative probability distributions for the upper- and

469 lower-bound time series of $\mathrm{NH}_{3}-\mathrm{N}$ loading at any emission level are Weibull and Gamma

470 distributions, respectively. Table 2 shows loading distributions and the associated probabilities for

$471 \mathrm{TP}$ and $\mathrm{NH}_{3}-\mathrm{N}$, respectively.

472

473 Place Table 2 and Figures 3 to 6 here

474

475

476

4.2 Optimal trading scheme

477

478 In this study, totally 9 scenarios based on each trading mechanism are examined considering 
479 three levels of water availability $(w)$ and three environmental allowance-violation risk levels $(p)$.

480 The trading ratio is introduced to ensure that the water quality between the trading sources is

481 equivalent. It is determined according to the hazard degree of pollutants produced by each

482 industry, the location of pollution sources and the water quality standard of the discharged

483 watershed.

In tables 3 to 6 , the results are provided in forms of "selling amount/purchasing amount". The selling and purchasing amount would be different because of trading ratio. Tables 3 and 4 show the detailed optimal trading schemes for TP and $\mathrm{NH}_{3}-\mathrm{N}$ permits when $p=0.01$ under Case 1 and $w=1$. Under Case 1, transactions are implemented across different reaches within same watershed. For example, Jiaozhou agricultural zone would purchase 79.7 ton of $\mathrm{NH}_{3}-\mathrm{N}$ permits from Laixi livestock and poultry industry zone (selling amount of 154.6 ton) $(w=1)$ under Case 1 as shown in Table 4. 74.9 ton of $\mathrm{NH}_{3}-\mathrm{N}$ permits would be eliminated in this trading section because of trading ratio. Tables 5 and 6 illustrate the detailed optimal trading schemes for TP and $\mathrm{NH}_{3}-\mathrm{N}$ permits when $p$ takes 0.01 under Case 2 and $w=1$. The transactions are implemented across different reaches and watersheds to satisfy the discharge allowance for all the sections. For example, Moshuihe Jimo agricultural zone would sell 134.08 ton of $\mathrm{NH}_{3}-\mathrm{N}$ permits to Pingdu livestock and poultry industry zone (purchasing amount of 134.08 ton) $(w=1)$ under Case 2 (Table 6). The detailed trading processes under $p=0.01$ in medium level of water availability are shown in Compressed File, Table S1-S4.

499 
503 The excess total TP and $\mathrm{NH}_{3}-\mathrm{N}$ emissions from agricultural zones, total $\mathrm{TP}$ and $\mathrm{NH}_{3}-\mathrm{N}$ emissions, 504 system net benefits and total trading amounts are investigated under the three environmental 505 allowance-violation risks $(p)$. Table 7 shows the total excess $\mathrm{TP}$ and $\mathrm{NH}_{3}-\mathrm{N}$ emissions from 506 agriculture at different $p$. The results indicate that generally the total excess TP and $\mathrm{NH}_{3}-\mathrm{N}$ 507 emissions from agriculture would be decreased as $p$ is increased except for the scenarios under no 508 excess TP and $\mathrm{NH}_{3}-\mathrm{N}$. Table 8 shows the total excess TP and $\mathrm{NH}_{3}-\mathrm{N}$ emissions at different $p$.

509 From the results, the total excess TP and $\mathrm{NH}_{3}-\mathrm{N}$ emissions would also be decreased as $p$ is 510 increased accordingly. For example, under Case 1 and $w=2$, the total excess TP emissions would 511 be decreased from 521.48 ton to 520.30 ton as $p$ is raised from 0.01 to 0.1 .Figure 7 shows the net 512 system benefits at different $p$. The results illustrate that net system benefits would be increased as $513 p$ is improved due to the decreased total excess $\mathrm{TP}$ and $\mathrm{NH}_{3}-\mathrm{N}$ emissions. For example, under

514 Case 1 and $w=2$, the net system benefits would be RMB $¥ 17947.415 \times 10^{6}(p=0.01)$ and 515 RMB $¥ 17947.578 \times 10^{6}(p=0.1)$. Figure 8 shows the total $\mathrm{TP}$ and $\mathrm{NH}_{3}-\mathrm{N}$ trading amounts at 516 different $p$. The results indicate that $\mathrm{TP}$ and $\mathrm{NH}_{3}-\mathrm{N}$ trading amounts under $p=0.01$ are higher 517 than those under $p=0.1$ because the decreased total excess pollution emissions would decrease 518 the desire for $\mathrm{TP}$ and $\mathrm{NH}_{3}-\mathrm{N}$ permits trading program. Above results imply that the total excess $519 \mathrm{TP}$ and $\mathrm{NH}_{3}-\mathrm{N}$ emissions from agricultural zones would be decreased when $p$ is raised. Then the 520 total excess TP and $\mathrm{NH}_{3}-\mathrm{N}$ emissions would be decreased, leading to the increased net system 521 benefits and decreased total trading amounts. 
526 The total trading amounts, excess total $\mathrm{TP}$ and $\mathrm{NH}_{3}-\mathrm{N}$ emissions and system net benefits are 527 investigated under three levels for water availability $(w)$. From the results in Figure 8, when the 528 level of water availability varies from low to high, the total $\mathrm{TP}$ and $\mathrm{NH}_{3}-\mathrm{N}$ trading amounts 529 would increase. For example, under Case 2 and when $p$ takes 0.01 , the $\mathrm{NH}_{3}-\mathrm{N}$ trading amounts 530 would be 54.87 ton and 1139.11 ton when $w=2$ and $w=1$, respectively. This is mainly because 531 the existence of demand and more supply for $\mathrm{TP}$ and $\mathrm{NH}_{3}-\mathrm{N}$ permits when the level of water 532 availability is high. The demand represents the existence of excess pollution emissions from many pollution sources which need to require more pollution permits. The supply represents that the total surplus TP and $\mathrm{NH}_{3}-\mathrm{N}$ permits would be increased by 0.76 ton and 1287.49 ton before trading when the level of water availability rises from low to high. This implies that the effluent trading would be promoted in high level of water availability. The total excess $\mathrm{TP}$ and $\mathrm{NH}_{3}-\mathrm{N}$ emissions would be decreased when the level of water availability is raised due to the increased total trading amounts (Table 8). For example, when $p=0.01$ under Case 2 , the total excess $\mathrm{NH}_{3}-\mathrm{N}$ emissions would be decreased from 1195.66 ton to 614.11 ton. What's more, the net system benefits would be increased accordingly (Figure 7). For example, when $p=0.01$ under Case 2, the net system benefits are RMB $17970.639 \times 10^{6}$ and RMB¥ $17947.415 \times 10^{6}$ under $w=$

5421 and $w=2$. Above the results indicate that the total trading amounts would be increased when the level of water availability is raised. After that, the total excess TP and $\mathrm{NH}_{3}-\mathrm{N}$ emissions 544 would be decreased and net system benefits would be increased. 
548 Firstly, from the results in Table 8, the total excess $\mathrm{TP}$ and $\mathrm{NH}_{3}-\mathrm{N}$ emissions under Cases 1 and 2

549

550

551

552

553

554

555

556

557

558

559

560

561

562

563

564

565

566

567

568

569

570

would be lower than those under Case 3 when $w=1$. For example, when $p=0.1$ and $w=1$, the total excess TP emissions under Cases 1, 2 and 3 are 238.63 ton, 231.96 ton and 240.82 ton.

Figure 9 depicts the amounts of the eliminated $\mathrm{TP}$ and $\mathrm{NH}_{3}-\mathrm{N}$ permits from trading market under three levels for water availability as well as Cases 1 and 2. The eliminated TP and $\mathrm{NH}_{3}-\mathrm{N}$ permits imply the reduced emission permits under Cases 1 or 2 over Case 3. From the results, there are some eliminated TP and $\mathrm{NH}_{3}-\mathrm{N}$ permits under Cases 1 and 2 when $w=1$. This leads to strict environmental allowances under Cases 1 and 2. In addition, from the results of Figure 7, the net system benefits under Cases 1 and 2 would be higher than those under Case 3 when $w=1$. For example, when $p=0.1$ and $w=1$, the net system benefits are RMB $¥ 17970.439 \times 10^{6}$ (Case 1 ), $17970.639 \times 10^{6}$ (Case 2), $17970.028 \times 10^{6}$ (Case 3). Based on the above results, trading cases perform better than non-trading case in high level of water availability. This is mainly because the trading cases can achieve the optimal configuration for pollution permits. Secondly, the total excess TP and $\mathrm{NH}_{3}-\mathrm{N}$ emissions under Cases 1 and 2 would be higher than those under Case 3 when $w=2$ (Table 8). For example, when $p=0.1$ and $w=2$, the total excess TP emissions under Cases 1, 2 and 3 are 520.30 ton, 520.30 ton and 519.05 ton. In addition, the increased amounts of total excess TP and $\mathrm{NH}_{3}-\mathrm{N}$ emissions under Case 1 and 2 compared with Case 3 are similar to the eliminated permits under Case 1 and 2. Furthermore, the net system benefits under Cases 1 and 2 would be lower than those under Case 3 when $w=2$ (Figure 7). For example, when $p=0.1$ and $w$ $=2$, the net system benefits are RMB¥ $17947.578 \times 10^{6}$ (Case 1), $17947.578 \times 10^{6}$ (Case 2), $17947.768 \times 10^{6}$ (Case 3). The above results imply that the system in low level of water availability is suitable for non-trading mechanism. This is mainly because there is almost no surplus pollution discharge permits for all pollution sources in low level of water availability. 
571 Thirdly, the total excess TP emissions under Cases 1 and 2 would be higher than those under

572 Case 3 when $w=3$ (Table 8). But total excess $\mathrm{NH}_{3}-\mathrm{N}$ emissions under Cases 1 and 2 would be

573 lower than those under Case 3. For example, when $p=0.1$ and $w=3$, the total excess $\mathrm{TP}\left(\mathrm{NH}_{3}-\mathrm{N}\right)$

574 emissions under Cases 1, 2 and 3 are 380.95 ton (732.82 ton), 372.88 ton (732.82 ton) and 370.46

575 ton (750.31 ton). In addition, from the results in Figure 9, there are some eliminated TP and

$576 \mathrm{NH}_{3}-\mathrm{N}$ permits under Cases 1 and 2 when $w=3$. This leads to strict environmental allowances

577 under Cases 1 and 2. Furthermore, the results indicate that the net system benefits under Cases 1

578 and 2 would be higher than those under Case 3 when $w=3$ (Figure 7). For example, when $p=0.1$

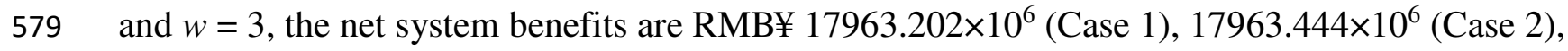

$58017963.080 \times 10^{6}$ (Case 3). The above results indicate that trading cases perform better than

581 non-trading case in medium level of water availability. This is mainly because trading cases can

582 achieve the optimal configuration for pollution permits.

583

$584 \quad$ Place Figure 9 here

585

586

587 From the results in Table 8, the total excess TP emissions under Case 1 would be increased by

$588[6.67,11.2]$ ton compared with Case 2 when $w=1$. This is mainly because certain surplus

589

pollution permits would be traded between pollution sources under cross-watershed trading case,

but they cannot be traded under intra-watershed trading. The results illustrate that the eliminated

591 TP permits under Case 1 would almost be equivalent to those under Case 2 when $w=1$ (Figure

592

9). The above results imply that the TP trading under Case 2 can better achieve pollution permits'

593 optimal configuration than Case 1. In addition, the total excess $\mathrm{NH}_{3}-\mathrm{N}$ emissions would be same 
594

595

596

597

598

599

600

601

602

603

604

605

606

607

608

609

610

611

612

613

614

615

under Cases 1 and 2 when $w=1$ (Table 8). However, the eliminated $\mathrm{NH}_{3}-\mathrm{N}$ permits under Case 1 would be increased by $[234.39,292.02]$ ton compared with Case 2 (Figure 9). This results in the stricter environmental allowance for $\mathrm{NH}_{3}-\mathrm{N}$ under Case 1 . This is mainly because the total agricultural $\mathrm{NH}_{3}-\mathrm{N}$ reallocation emission permits under case 1 would be higher than Case 2 when the total agricultural excess $\mathrm{NH}_{3}-\mathrm{N}$ emissions are all 0 ton with the same total agricultural $\mathrm{NH}_{3}-\mathrm{N}$ emissions under Cases 1 and 2. This leads to [321.22, 335.76] ton of surplus $\mathrm{NH}_{3}-\mathrm{N}$ permits under Case 2. Therefore, the above results imply that $\mathrm{NH}_{3}-\mathrm{N}$ trading under intra-watershed trading case performs better than cross-watershed trading case. From the results in Figure 7, the net system benefits under Case 2 would be higher than those under Case 1 when $w=1$. For example, when $p=0.01$ and $w=1$, the net system benefits under Cases 1 and 2 would be RMB¥ $17970.303 \times 10^{6}$ and RMB¥ $17970.639 \times 10^{6}$. All the above results imply that cross-watershed trading case would be better than intra-watershed trading case due to the increased net system benefits. But the environmental allowance for $\mathrm{NH}_{3}-\mathrm{N}$ is stricter under intra-watershed trading case than cross-watershed trading case. From the results in Table 8, the total excess TP emissions under Case 1 would be increased by $[2.65,8.06]$ ton compared with Case 2 when $w=3$. This is mainly because the certain surplus pollution permits would be traded between pollution sources under cross-watershed trading case, but they cannot be traded under intra-watershed trading. In addition, the results illustrate that the eliminated TP permits under Case 1 would almost be equivalent to those under Case 2 when $w=3$ (Figure 9). The above results imply the TP trading under Case 2 that can better obtain the optimal configuration for pollution permits. In addition, the total excess $\mathrm{NH}_{3}-\mathrm{N}$ emissions would be equivalent under Cases 1 and 2 when $w=3$ (Table 8). And the eliminated $\mathrm{NH}_{3}-\mathrm{N}$ permits under Case 1 would be 
616 decreased by $[4.43,7.73]$ ton compared with Case 2 when $w=3$ (Figure 9). This leads to the 617 stricter environmental allowance for $\mathrm{NH}_{3}-\mathrm{N}$ under Case 2. The above results imply that the 618 optimal configuration for pollution permit of the trading for $\mathrm{NH}_{3}-\mathrm{N}$ under cross-watershed 619 trading case is better than intra-watershed trading case in medium level of water availability. The 620 net system benefits under Case 2 would be higher than those under Case 1 when $w=3$. For example, when $p=0.01$ and $w=3$, the net system benefits would be RMB¥ $17963.282 \times 10^{6}$

622 (Case 1)and RMB¥ $17963.595 \times 10^{6}$ (Case 2). All the above results imply that the trading under cross-watershed trading case would be better in optimal configuration for pollution permit due to the increased net system benefits. Meanwhile, the environmental allowance for TP and $\mathrm{NH}_{3}-\mathrm{N}$ is stricter under cross-watershed trading case than intra-watershed trading case.

Figure 10 shows the $\mathrm{TP}$ and $\mathrm{NH}_{3}-\mathrm{N}$ detailed trading processes for agriculture, livestock and poultry industry, fishery and company under Cases 1 and 2. From the results, Firstly, under Case 1, the main selling (purchasing) industries for TP permits are agriculture (fishery), livestock and poultry industry (agriculture) and livestock and poultry industry (agriculture) under the three $w$, respectively. And the main selling (purchasing) industries for $\mathrm{NH}_{3}-\mathrm{N}$ permits are agriculture (livestock and poultry industry), livestock and poultry industry (agriculture) and agriculture (livestock and poultry industry) for the three $w$. For example, under $p=0.01$ and when $w=1$, the $\mathrm{TP}\left(\mathrm{NH}_{3}-\mathrm{N}\right)$ trading amounts for agriculture, livestock and poultry industry, fishery and company would be 27.83 ton (392.17 ton), 7.44 ton (-75.53 ton), -2.76 ton ( -34.47 ton) and 0.25 ton (1.29 ton) under Case 1. Secondly, agriculture (livestock and poultry industry) is the main selling 
637 (purchasing) industry for $\mathrm{TP}$ and $\mathrm{NH}_{3}-\mathrm{N}$ permits in the trade under Case 2 for the three $w$, except 638 for $\mathrm{NH}_{3}-\mathrm{N}$ permits when $w=1$.

639

640 Place Figure 10 here

641

642

643 From the results in Figure 8, the total $\mathrm{TP}$ and $\mathrm{NH}_{3}-\mathrm{N}$ trading amounts under Case 2 would

644 generally be higher than those under Case 1, except for $\mathrm{NH}_{3}-\mathrm{N}$ trading amount when $w=1$. For

645 example, when $p$ takes 0.01 and $w=2$, the total $\mathrm{TP}$ and $\mathrm{NH}_{3}-\mathrm{N}$ trading amounts would be 8.97

646

ton and 36.06 ton under Case 1; the total $\mathrm{TP}$ and $\mathrm{NH}_{3}-\mathrm{N}$ trading amounts would be 21.29 ton and

64754.87 ton under Case 2. This is mainly because when cross-watershed effluent trading (Case 2) is

648 allowed, the permits in pollution sources from Daguhe watershed can be sold to other sources

649 from Moshuihe watershed. This implies that cross-watershed effluent trading can motivate the 650 market's vitality.

651

652

5. Conclusions

653

654 In this study, a Bayesian simulation-based multi-watershed effluent trading designing model

655 (BS-METM) has been proposed to support multi-watershed nonpoint source pollution

656

management. BS-METM combines Bayesian estimation with DREAM, nutrient fate modeling by

657

using SWAT, optimal design for effluent trading and mechanism analysis within a framework.

658

BS-METM has several advantages in: (i) estimating the uncertainties of parameters in modeling

659 fate of nutrients from nonpoint sources through Bayesian inference; (ii) disclosing the detailed 
660

661

662

663

664

665

666

667

668

669

670

671

672

673

674

675

676

677

678

679

680

681

optimal effluent trading schemes considering multiple uncertainties as well as system risk; (iii)

revealing the influences of vital factors on the trading process (i.e. environmental

allowance-violation risk level and water availability level); (iv) identifying optimal effluent

trading mechanism through revealing interaction among trading processes of multiple watersheds.

BS-METM has been applied to multi-watershed effluent trading design for two agricultural watersheds, i.e., Daguhe and Moshuihe watersheds, China, where TP and $\mathrm{NH}_{3}-\mathrm{N}$ are selected as water quality indicators. The optimized industry scales and trading processes are obtained. The effects of important factors on the trading process (i.e. environmental allowance-violation risk level and water availability level) are investigated. The interactions between water availability level and trading mechanism are also analyzed. Several results are revealed: (i) the increased level of environment allowance leads to the decreased total excess $\mathrm{TP}$ and $\mathrm{NH}_{3}-\mathrm{N}$ emissions and trading amounts as well as the increased net system benefits; the net system benefits would be increased by $\mathrm{RMB} ¥[0,227] \times 10^{3}$ with environmental allowance-violation risk level; (ii) the total trading amounts should be enlarged under high water availability level because of the existence of demand and more supply for TP and $\mathrm{NH}_{3}-\mathrm{N}$ permits; high water availability level also corresponds to high net system benefits; (iii) the total $\mathrm{TP}$ and $\mathrm{NH}_{3}-\mathrm{N}$ trading amounts under cross-watershed trading would be generally higher than those under intra-watershed trading; this implies that cross-watershed effluent trading can motivate the trading market's vitality; (iv) the trading cases would be recommended due to the optimal configuration for pollution permit in medium and high levels of water availability; and the net system benefits under trading cases 
682 would increase by RMB¥ $[47,611] \times 10^{3}$ compared with non-trading case; but non-trading case 683 would be recommended in low water availability level because there is almost no surplus 684 pollution discharge permits in the region; (v) in medium and high water availability level, 685 cross-watershed trading case would be recommended compared with intra-watershed trading case, 686 with increased net system benefits of $\operatorname{RMB} ¥[200,336] \times 10^{3}$ and $[80,242] \times 10^{3}$ for the two 687 levels; strict environmental management of $\mathrm{NH}_{3}-\mathrm{N}$ should be strengthened due to less eliminated 688 discharge permits under cross-watershed trading. 


\section{Acknowledgement}

691

692 This research was supported by the National Natural Science Foundation of China (51809145, 693 42007412), Shandong Key Laboratory of Water Pollution Control and Resource Reuse (Grant No.

694 2019KF10), Research on the Measurement and Optimization of Marine Resources Carrying 695 Capacity from the Perspective of Biased Technological Progress (No.18YJC630152).

696 
The assessment of SWAT model parameters contains uncertainties originating from errors and spatiotemporal heterogeneity, which may encounter difficulties in accurately depicting nutrient fate with an unrealistic estimation of parameter uncertainty. MCMC will provide a valid way that would account for parameter uncertainty in a Bayesian inference. The Bayesian theorem can be showed as follows (Zhang et al., 2019):

704

$$
f_{\text {post }}\left(\theta \mid y_{\text {obs }}\right)=\frac{f_{p r i}(\theta) \cdot f_{M}\left(y_{o b s} \mid \theta\right)}{\int f_{p r i}(\theta) \cdot f_{M}\left(y_{o b s} \mid \theta\right) d \theta}
$$

where $f_{\text {post }}\left(\theta \mid y_{\text {obs }}\right)$ represents the posterior distribution of parameter set $\theta$ that is dated from the prior distribution $f_{p r i}(\theta)$ conditioned on observed data $y_{o b s} ; f_{M}\left(y_{o b s} \mid \theta\right)$ is the likelihood function. In the study, the simulation errors are assumed to be independent and identically normally distributed, which determines the construction of the likelihood function and is the basis of the entire Bayesian calibration process. The likelihood function is (Raje and Krishnan, 2012):

711

$$
f_{M}\left(y_{o b s} \mid \theta_{M}\right)=\prod_{t} \frac{1}{\sqrt{2 \Pi \sigma_{e}}} \exp \left[-\frac{\left(y_{t}-y_{o b s, t}\right)^{2}}{2 \sigma_{e}^{2}}\right]
$$
where $y_{t}$ is the simulated nutrient loading with SWAT at time step $t ; y_{o b s, t}$ is the observed data at time step $t ; \sigma_{e}^{2}$ is the variance of the simulation errors. 
717 in surface runoff, which is simulated based on spatial information on climate, topography, soil

718 properties, land use and management practices. The process simulation of nitrogen $(\mathrm{N})$ in soil can

719 be divided into two main parts, including nitrate transport and organic nitrogen $\mathrm{N}$ loss. Nitrate is

720 transported by dissolving in surface runoff, lateral flow, or percolation. The nitrate concentration

721 in mobile water can be calculated by the following equation:

722

723

$$
\begin{aligned}
& w_{3 \text { surf }}=Q_{\text {surf }}+Q_{\text {lat }, l y}+w_{\text {perc }, l y} \\
& N O_{3 \text { surf }}=\frac{\beta_{N O_{3}} Q_{\text {surf }} N O_{3_{l y}}\left\{1-\exp \left[-w_{\text {mobile }} /\left(1-\theta_{e}\right) S A T_{l y}\right]\right\}}{w_{\text {mobile }}}
\end{aligned}
$$

724

725 where $\mathrm{NO}_{3 \text { surf }}$ is the nitrate removed in surface runoff $(\mathrm{kg} / \mathrm{ha}) ; \beta_{\mathrm{NO}_{3}}$ represents the nitrate percolation coefficient; $\mathrm{NO}_{3_{l y}}$ is the amount of nitrate in the soil layer $(\mathrm{kg} / \mathrm{ha}) ; w_{\text {mobile }}$ represents the amount of mobile water in the layer $\left(\mathrm{mm} \mathrm{H}_{2} \mathrm{O}\right) ; \theta_{e}$ is the fraction of porosity from which anions are excluded; $S A T_{l y}$ is the water content of the soil layer; $Q_{l a t, l y}$ represents the water discharged from the layer by lat-eral flow $\left(\mathrm{mm} \mathrm{H}_{2} \mathrm{O}\right)$; and $w_{\text {perc, }, y}$ denotes the amount of water percolating to the underlying soil layer $\left(\mathrm{mm} \mathrm{H}_{2} \mathrm{O}\right)$. The organic $\mathrm{N}$ runoff loss based on the organic $\mathrm{N}$ concentration in the top soil layer and the sediment yield can be calculated by using the following equation:

$$
\operatorname{org} N_{\text {surf }}=\frac{0.001 \operatorname{conc}_{\text {orgN }} \varepsilon_{\text {sedN }} \text { sed }}{\text { area }_{\text {hru }}}
$$

735 where $\operatorname{org} N_{\text {surf }}$ is the amount of organic $\mathrm{N}$ transported to the chief channel in surface runoff 
$736(\mathrm{~kg} / \mathrm{ha}) ;$ conc $_{\text {org }}$ denotes the organic $\mathrm{N}$ concentration in the top $10 \mathrm{~mm}(\mathrm{~g} / \mathrm{kg}) ;$ area $_{\text {hru }}$ is the 737 HRU area (ha); and $\varepsilon_{\text {sedv }}$ represents the $\mathrm{N}$ enrichment ratio. SWAT simulates the dynamics of 738 three forms of phosphorous $(\mathrm{P})$, including organic $\mathrm{P}$ which exists in humus, insoluble mineral $\mathrm{P}$ 739 and soluble $\mathrm{P}$. The amount of organic $\mathrm{P}$ transported with sediment to the stream is simulated with 740 a loading function as depicted in organic $\mathrm{N}$ as follows:

741

$$
\operatorname{org} P_{\text {surf }}=0.001 \text { conc }_{\text {orgP }} \varepsilon_{\text {sedP }} \text { sed } / \text { area }_{\text {hru }}
$$

742

743

where $\operatorname{org} P_{\text {sur }}$ is the amount of organic $\mathrm{P}$ transported to the main channel in surface runoff

$(\mathrm{kg} / \mathrm{ha}) ; \operatorname{conc}_{\text {orgP }}$ denotes the organic P concentration in the top $10 \mathrm{~mm}(\mathrm{~g} / \mathrm{kg}) ;$ and $\varepsilon_{\text {sedP }}$

represents the P enrichment ratio. Because of the low mobility of soluble P, SWAT only considers the loss of the soluble $\mathrm{P}$ with surface runoff based on labile $\mathrm{P}$ concentration in the top soil layer.

The migration of soluble $\mathrm{P}$ in surface runoff is:

$$
P_{\text {surf }}=\frac{10 P_{\text {soluble, surf }} Q_{\text {surf }}}{\rho_{b} k_{d, \text { surf }}}
$$

where $P_{\text {surf }}$ represents the amount of soluble P lost in surface runoff $(\mathrm{kg} / \mathrm{ha}) ; P_{\text {soluble,surf }}$ is the amount of soluble $\mathrm{P}$ in the top $10 \mathrm{~mm}(\mathrm{~kg} / \mathrm{ha}) ; \rho_{b}$ denotes the bulk density of the top $10 \mathrm{~mm}$ $\left(\mathrm{Mg} / \mathrm{m}^{3}\right)$; and $k_{d, \text { surf }}$ the $\mathrm{P}$ soil partitioning coefficient $\left(\mathrm{m}^{3} / \mathrm{Mg}\right)$. 
Agricultural zone, $i=1,2,3,4,5 ; i=1$ for Laixi zone, $i=2$ for Pingdu zone, $i=3$ for Jiaozhou zone, $i=4$ for Daguhe Jimo zone, $i=5$ for Moshuihe Jimo zone. Species of crops, $j=1,2,3,4, \ldots, 11 ; j=1$ for wheat, $j=2$ for corn, $j=3$ for peanut, $j=4$ for chinese cabbage, $j=5$ for celery, $j=6$ for carrot, $j=7$ for potato, $j=8$ for apple, $j=9$ for pear, $j=10$ for peach, $j=11$ for grape.

$n$
Livestock and poultry industry zone, $n=1,2,3 ; n=1$ for Laixi zone, $n=2$ for Pingdu zone, $n=3$ for Jiaozhou zone.

Species of livestocks, $r=1,2,3,4 ; r=1$ for chicken, $r=2$ for pig, $r=3$ for cattle, $r=4$ for cow.

Fishery zone, $p=1,2,3 ; p=1$ for Laixi zone, $p=2$ for Pingdu zone, $p=3$ for Jiaozhou zone

$$
\text { company, } m=1,2,3,4,5,6,7 ; m=1
$$

for Qingdao Zhengyuan Iron and Steel Co., Ltd $m=2$ for Qingdao Tongyuanchang Steel Co., Ltd $m=3$ for Qingdao Hehe Chemical Co., Ltd $m=4$ for Qingdao Zeyukaisheng Machinery Manufacturing Co., Ltd $m=5$ for Qingdao Huataida Machinery Manufacturing Co., Ltd $m=6$ for Qingdao Jingrui Machinery Manufacturing Co., Ltd $m=7$ for Qingdao Jinguangxin Textile Co., Ltd Other agricultural zone except zone $i$ Other livestock and poultry industry zone except zone $n$

Other species of livestocks except $r$ 


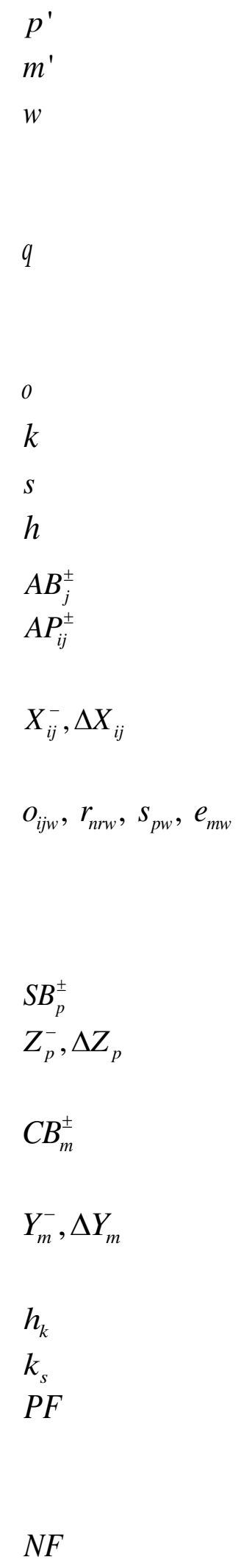

Other fishery zone except zone $p$

Other company except company $m$

The level of water availability, $w=1,2,3$;

$w=1$ for high level, $w=2$ for low level,

$w=3$ for medium level.

Reaches in Daguhe watershed, $q=1,2,3,4$;

$q=1$ for Laixi reach, $q=2$ for Pingdu reach,

$q=3$ for Jiaozhou reach, $q=4$ for Jimo reach.

Reaches in Moshuihe watershed, $o=1,2$

TP-generation level of agriculture

$\mathrm{NH}_{3}-\mathrm{N}$-generation level of agriculture

A prescribed level of probability for each constraint

Net benefit of crop j (RMB¥/ha)

Production level of crop $j$ in agricultural zone $i$ (kg/ha)

Lower bound and range of area target for crop $\mathrm{j}$ in zone $i$ (ha)

Decision variables which are used for identifying the optimized targets of cropped area, the scale of livestock and poultry industry, the scale of fishery and the production level of company

Net benefit of zone $p \quad(\mathrm{RMB} ¥ / \mathrm{ha})$

Lower bound and range of the scale of fishery zone $p$

Net benefit of per unit product of company $m$

(RMB¥)

Lower bound and range of the production level of company $m$

Probability of TP generation rate in agriculture

Probability of $\mathrm{NH}_{3}-\mathrm{N}$ generation rate in agriculture Penalties per ton excess TP effluents exceeding to discharge permits from pollution source

(RMB¥/ton)

Penalties per ton excess $\mathrm{NH}_{3}-\mathrm{N}$ effluents exceeding 


$$
E D P A_{i k w}^{ \pm}, E D P R_{n w}^{ \pm}, E D P P_{p w}^{ \pm}, E D P C_{m w}^{ \pm}
$$$$
E D N A_{i s w}^{ \pm}, E D N R_{n w}^{ \pm}, E D N P_{p w}^{ \pm}, E D N C_{m w}^{ \pm}
$$

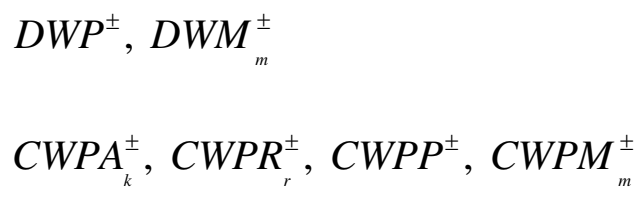

to discharge permits from pollution source

(RMB¥/ton)

Excess annual TP loading for agricultural zone $i$, livestock and poultry industry zone $n$, fishery zone $p$ and company $m$ (ton)

Excess annual $\mathrm{NH}_{3}-\mathrm{N}$ loading for agricultural zone $\mathrm{i}$, livestock and poultry industry zone $n$, fishery zone $p$ and company $m$ (ton)

Effluent generation rate of fishery zone and company $m\left(\mathrm{~m}^{3} / \mathrm{ha} ; \mathrm{m}^{3} /\right.$ ton, item $)$

TP generation rate of agricultural zone $i$, livestock $r$, fishery zone $p$ and company $m$ (ton/ha; ton $/$ item; ton $/ \mathrm{m}^{3}$; ton $/ \mathrm{m}^{3}$ )

$\mathrm{NH}_{3}-\mathrm{N}$ generation rate of agricultural zone $i$, livestock $r$, fishery zone $p$ and company $m$ (ton/ha; ton/item; ton $/ \mathrm{m}^{3}$; ton $/ \mathrm{m}^{3}$ )

TP discharge permit allocated to agricultural zone $i$, livestock and poultry industry $n$, fishery zone $p$ and company $m$ in level $w$, respectively (ton)

$\mathrm{NH}_{3}-\mathrm{N}$ discharge permit allocated to agricultural zone $i$, livestock and poultry industry $n$, fishery zone $p$ and company $m$ in level $w$, respectively (ton)

TP discharge permit that agricultural $i$, livestock and poultry industry zone $n$, fishery zone $p$ and company $m$ possess after trading program in level $w$ (ton)

$\mathrm{NH}_{3}-\mathrm{N}$ discharge permit that agricultural $i$, livestock and poultry industry zone $n$, fishery zone $p$ and company $m$ possess after trading program in level $w$ (ton)

TP discharge permit sold to agricultural zone $i$ from agricultural zone $i^{\prime}$, livestock and poultry 
$T P_{n^{\prime} n w}, T P s_{i n w}, T P s_{p n w}, T P s_{m n w}$

$T P_{p^{\prime} p w}, T P s_{i p w}, T P s_{n p w}, T P s_{m p w}$

$T P_{m^{\prime} m w}, T P s_{i m w}, T P s_{n m w}, T P s_{p m w}$

$T P b_{\text {niw }}, T P b_{\text {piw }}, T P b_{\text {miw }}$

$T P b_{i n w}, T P b_{p n w}, T P b_{m n w}$

$T P b_{i p w}, T P b_{n p w}, T P b_{m p w}$

$T P b_{i m w}, T P b_{n m w}, T P b_{p m w}$

$T P_{i i^{\prime} w}, T P_{n n^{\prime} w}, T P_{p p^{\prime} w}, T P_{m m^{\prime} w}$ industry zone $n$, fishery zone $p$ and company

$m$, respectively (ton)

TP discharge permit sold to livestock and poultry industry zone $n$ from livestock and poultry industry zone $n^{\prime}$, agricultural zone $i$, fishery zone $p$ and company $m$, respectively (ton) TP discharge permit sold to fishery zone $p$ from fishery zone $p^{\prime}$, agricultural zone $i$, livestock and poultry industry zone $n$ and company $m$, respectively (ton)

TP discharge permit sold to company $m$ from company $m^{\prime}$, agricultural zone $i$, livestock and poultry industry zone $n$ and fishery zone $p$, respectively (ton)

TP discharge permit in agricultural zone $i$ purchased from livestock and poultry industry zone $n$, fishery zone $p$ and company $m$, respectively (ton)

TP discharge permit in livestock and poultry industry zone $n$ purchased from agricultural zone $i$, fishery zone $p$ and company $m$, respectively (ton)

TP discharge permit in fishery zone $p$ purchased from agricultural zone $i$, livestock and poultry industry zone $n$ and company $m$, respectively (ton)

TP discharge permit in company $m$ purchased from agricultural zone $i$, livestock and poultry industry zone $n$ and fishery zone $p$, respectively (ton)

TP discharge permit agricultural zone $i$ sold to $i^{\prime}$, livestock and poultry industry zone $n$ sold to $n^{\prime}$, fishery zone $p$ sold to $p^{\prime}$ and company $m$ sold to $m^{\prime}$, respectively (ton) 


$$
\begin{aligned}
& T N_{i^{\prime} i w}, T N s_{n i w}, T N s_{p i w}, T N s_{m i w} \\
& T N_{n^{\prime} n w}, T N s_{i n w}, T N s_{p n w}, T N s_{m n w} \\
& T N_{p^{\prime} p w}, T N s_{i p w}, T N s_{n p w}, T N s_{m p w} \\
& T N_{m^{\prime} m w}, T N s_{i m w}, T N s_{n m w}, T N s_{p m w}
\end{aligned}
$$$$
T N b_{\text {niw }}, T N b_{\text {piw }}, T N b_{\text {miw }}
$$

$$
T N b_{i n w}, T N b_{p n w}, T N b_{m n w}
$$$$
T N b_{i p w}, T N b_{n p w}, T N b_{m p w}
$$

$$
T N b_{i m w}, T N b_{n m w}, T N b_{p m w}
$$$$
T N_{i i^{\prime} w}, T N_{n n^{\prime} w}, T N_{p p^{\prime} w}, T N_{m m^{\prime} w}
$$

$\mathrm{NH}_{3}-\mathrm{N}$ discharge permit sold to agricultural zone $i$ from agricultural zone $i$, livestock and poultry industry zone $n$, fishery zone $p$ and company $m$, respectively (ton)

$\mathrm{NH}_{3}-\mathrm{N}$ discharge permit sold to livestock and poultry industry zone $n$ from livestock and poultry industry zone $n^{\prime}$, agricultural zone $i$, fishery zone $p$ and company $m$, respectively (ton)

$\mathrm{NH}_{3}-\mathrm{N}$ discharge permit sold to fishery zone $p$ from fishery zone $p^{\prime}$, agricultural zone $i$, livestock and poultry industry zone $n$ and company $m$, respectively (ton) $\mathrm{NH}_{3}-\mathrm{N}$ discharge permit sold to company $m$ from company $m^{\prime}$, agricultural zone $i$, livestock and poultry industry zone $n$ and fishery zone $p$, respectively (ton)

$\mathrm{NH}_{3}-\mathrm{N}$ discharge permit in agricultural zone $i$ purchased from livestock and poultry industry zone $n$, fishery zone $p$ and company $m$, respectively (ton)

$\mathrm{NH}_{3}-\mathrm{N}$ discharge permit in livestock and poultry industry zone $n$ purchased from agricultural zone $i$, fishery zone $p$ and company $m$, respectively (ton)

$\mathrm{NH}_{3}-\mathrm{N}$ discharge permit in fishery zone $p$ purchased from agricultural zone $i$, livestock and poultry industry zone $n$ and company $m$, respectively (ton)

$\mathrm{NH}_{3}-\mathrm{N}$ discharge permit in company $m$ purchased from agricultural zone $i$, livestock and poultry industry zone $n$ and fishery zone $p$, respectively (ton)

$\mathrm{NH}_{3}-\mathrm{N}$ discharge permit agricultural zone $i$ sold 
$t p_{i^{\prime} i}, t p_{n i}, t p_{p i}, t p_{m i}$

$t p_{n^{\prime} n}, t p_{i n}, t p_{p n}, t p_{m n}$

$t p_{p^{\prime} p}, t p_{i p}, t p_{n p}, t p_{m p}$

$t p_{m^{\prime} m}, t p_{i m}, t p_{n m}, t p_{p m}$

$t p_{i i^{\prime}}, t p_{n n^{\prime}}, t p_{p p^{\prime}}, t p_{m m^{\prime}}$

$t n_{i^{\prime} i}, t n_{n i}, t n_{p i}, t n_{m i}$

$t n_{n^{\prime} n}, t n_{i n}, t n_{p n}, t n_{m n}$

$t n_{p^{\prime} p}, t n_{i p}, t n_{n p}, t n_{m p}$ to $i$, livestock and poultry industry zone $n$ sold

to $n^{\prime}$, fishery zone $p$ sold to $p^{\prime}$ and company $m$ sold to $m^{\prime}$, respectively (ton)

TP trading ratio of transaction from agricultural zone $i^{\prime}$, livestock and poultry industry zone $n$, fishery zone $p$ and company $m$ to agricultural zone $i$, respectively

TP trading ratio of transaction from livestock and poultry industry zone $n^{\prime}$, agricultural zone $i$, fishery zone $p$ and company $m$ to livestock and poultry industry zone $n$, respectively TP trading ratio of transaction from fishery zone $p^{\prime}$, agricultural zone $i$, livestock and poultry industry zone $n$ and company $m$ to fishery zone $p$, respectively

TP trading ratio of transaction from company $m^{\prime}$, agricultural zone $i$, livestock and poultry industry zone $n$ and fishery zone $p$ to company $m$, respectively

TP trading ratio of transaction from agricultural zone $i$ to $i^{\prime}$, from livestock and poultry industry zone $n$ to $n^{\prime}$, from fishery zone $p$ to $p^{\prime}$ and from company $m$ to $m^{\prime}$, respectively $\mathrm{NH}_{3}-\mathrm{N}$ trading ratio of transaction from agricultural zone $i^{\prime}$, livestock and poultry industry zone $n$, fishery zone $p$ and company $m$ to agricultural zone $i$, respectively

$\mathrm{NH}_{3}-\mathrm{N}$ trading ratio of transaction from livestock and poultry industry zone $n^{\prime}$, agricultural zone $i$, fishery zone $p$ and company $m$ to livestock and poultry industry zone $n$, respectively $\mathrm{NH}_{3}-\mathrm{N}$ trading ratio of transaction from fishery zone $p^{\prime}$, agricultural zone $i$, livestock and poultry industry zone $n$ and company $m$ to 


$$
t n_{m^{\prime} m}, t n_{i m}, t n_{n m}, t n_{p m}
$$$$
t n_{i i^{\prime}}, t n_{n n^{\prime}}, t n_{p p^{\prime}}, t n_{m m^{\prime}}
$$

$p_{h}$

$P E_{r}$

$L E_{r}$

$D P$

$M P$

$N E$

$C E_{j}$

$L P_{r}$

$N P$

$T P A_{i w}, T P L_{n w}, T P S_{p w}, T P C_{m w}$

$T N A_{i w}, T N L_{n w}, T N S_{p w}, T N C_{m w}$

$T P G_{w}$ fishery zone $p$, respectively

$\mathrm{NH}_{3}-\mathrm{N}$ trading ratio of transaction from company

$m^{\prime}$, agricultural zone $i$, livestock and poultry

industry zone $n$ and fishery zone $p$ to company

$m$, respectively

$\mathrm{NH}_{3}-\mathrm{N}$ trading ratio of transaction from

agricultural zone $i$ to $i^{\prime}$, from livestock and

poultry industry zone $n$ to $n^{\prime}$, from fishery zone

$p$ to $p^{\prime}$ and from company $m$ to $m^{\prime}$,

respectively

Constraint-violation probability

The quantity of energy in per kg of crop $j$

$(\mathrm{kcal} / \mathrm{kg})$

The quantity of required energy in per livestock $r$

(kcal/one)

Total population in Daguhe watershed

Total population in Moshuihe watershed

The quantity of required energy for per person

from the crop in each year (kcal/one/a)

The quantity of digestible protein in per kg of crop j $(\mathrm{g} / \mathrm{kg})$

The quantity of required digestible protein for per livestock $r$ in each year (g/one/a)

The quantity of required digestible protein for per person $(\mathrm{g} / \mathrm{one} / \mathrm{a})$

Allowance of TP emission for agricultural zones, livestock and poultry industry zones, fishery zones and companies within the two watersheds in level $w$ (ton)

Allowance of $\mathrm{NH}_{3}-\mathrm{N}$ emission for agricultural zones, livestock and poultry industry zones, fishery zones and companies within the two watersheds in level $w$ (ton)

Allowance of TP emission in Daguhe watershed in 
level $w$ (ton)

$T N G_{w}$

$T P G F_{q w}$

$T N G F_{q w}$

$T P W_{w}$

$T N W_{w}$

$T P W F_{o w}$

$T N W F_{\text {ow }}$

$T P T_{w}$

$\mathrm{TNT}_{w}$
Allowance of $\mathrm{NH}_{3}-\mathrm{N}$ emission in Daguhe watershed in level $w$ (ton)

Allowance of TP emission in reach q, Daguhe watershed in level $w$ (ton)

Allowance of $\mathrm{NH}_{3}-\mathrm{N}$ emission in reach q, Daguhe watershed in level $w$ (ton)

Allowance of TP emission in Moshuihe watershed in level $w$ (ton)

Allowance of $\mathrm{NH}_{3}-\mathrm{N}$ emission in Moshuihe watershed in level $w$ (ton)

Allowance of TP emission in reach o, Moshuihe watershed in level $w$ (ton)

Allowance of $\mathrm{NH}_{3}-\mathrm{N}$ emission in reach o, Moshuihe watershed in level $w$ (ton)

Allowance of TP emission in both watersheds in level $w$ (ton)

Allowance of $\mathrm{NH}_{3}-\mathrm{N}$ emission in both watersheds in level $w$ (ton) 


\section{References}

Alam MJ, Dutta D (2012) A process-based and distributed model for nutrient dynamics in river basin: Development, testing and applications. Ecological Modeling 247:12-124. https://doi.org/10.1016/j.ecolmodel.2012.07.031.

Alnahit AO, Mishra AK, Khan A A (2020) Quantifying climate, streamflow, and watershed control on water quality across Southeastern US watersheds. Science of The Total Environment 739:139945. https://doi.org/ 10.1016/j.scitotenv.2020.139945.

Chahor Y, Casalí J, Giménez R, Bingner RL, Campo MA, Goñi M (2014) Evaluation of the AnnAGNPS model for predicting runoff and sediment yield in a small Mediterranean agricultural watershed in Navarre (Spain). Agricultural Water Management 134:24-37. https://doi.org/10.1016/j.agwat.2013.11.014.

Chen L, Han ZX, Li S, Shen ZY (2016) Framework design and influencing factor analysis of a water environmental functional zone-based effluent trading system. Environmental Management 58:645-654. https://doi.org/10.1007/s00267-016-0747-6.

Chen XJ, Sun HL, Xu HF (2019) Discrete Approximation of Two-Stage Stochastic and Distributionally Robust Linear Complementarity Problems. Mathematical Programming 177:255-289. https://doi.org/10.1007/s10107-018-1266-4.

Chen YY, Yang SY, Dong SJ, Li YM, Sun BQ, Shao ZJ (2010) Influence of Agricultural Activity and Aquifer Intrinsic Vulnerability on Groundwater Quality in the Dagu River Watershed (Qingdao, China). 2010 4th International Conference on Bioinformatics and Biomedical Engineering, (ICBBE 2010). https://doi.org/10.1109/ICBBE.2010.5517811. 
Clark DL, Keil DP, Murray MR, Neethling J, Rawls NB, Moss D (2008) Water quality trading and advanced treatment technology to meet the lowest phosphorus requirements in the nation. Proceedings of the Water Environment Federation 16:967-988. https://doi.org/10.2175/193864708788735303.

Corrales J, Naja GM, Bhat MG, Miralles-Wilhelm F (2017) Water quality trading opportunities in two sub-watersheds in the northern Lake Okeechobee watershed. Journal of Environmental Management 196:544-559. https://doi.org/10.1016/j.jenvman.2017.03.061.

Huang GH, Loucks DP (2000) An inexact two-stage stochastic programming model for water resources management under uncertainty. Civil Engineering and Environmental Systems 17:95-118. https://doi.org/10.1080/02630250008970277.

Hung MF, Shaw D (2005) A trading-ratio system for trading water pollution discharge permits. Journal of Environmental Economics and Management 49:83-102. https://doi.org/10.1016/j.jeem.2004.03.005.

Laloy E, Rogiers B, Vrugt JA, Mallants D, Jacques D (2013) Efficient posterior exploration of a high-dimensional groundwater model from two-stage Markov chain Monte Carlo simulation and polynomial chaos expansion. Water Resources Research 49:2664-2682. https://doi.org/10.1002/wrcr.20226.

Li L, Liang SK, Shi XY, Wang XL (2009) Contaminative Conditions Analysis of Main Rivers Flowing into Jiaozhou Bay in 2007. Environmental Science and Management 6:27-32.

Li YP, Huang GH (2008) Interval-parameter two-stage stochastic nonlinear programming for water resources management under uncertainty. Water Resources Management 22:681-698. https://doi.org/10.1007/s11269-007-9186-8. 
Li YP, Huang GH, Nie SL (2006) An interval-parameter multi-stage stochastic programming model for water resources management under uncertainty. Advances in Water Resources 29:776-789. https://doi.org/10.1016/j.advwatres.2005.07.008.

Li YP, Huang GH, Nie SL, Liu L (2008) Inexact multistage stochastic integer programming for water resources management under uncertainty. Journal of Environmental Management 88:93-107. https://doi.org/10.1016/j.jenvman.2007.01.056.

Liao KH, Xu SH, Cheng GF, Lin Q (2010) Influencing factors and cokriging spatial interpolation analysis of soil cation exchange capacity - a case study of Dagu River Basin, Qingdao city. Acta Pedologica Sinica 47:26-32.

Liu J, Nie S, Li YP, Huang GH (2016) Two-stage vertex analysis method for planning electric power systems with greenhouse gas abatement consideration. International Journal of Green Energy 13:1000-1015. https://doi.org/10.1080/15435075.2014.974761.

Maeda S, Kawachi T, Unami K, Takeuchi J, Izumi T, Chono S (2009) Fuzzy optimization model for integrated management of total nitrogen loads from distributed point and nonpoint sources in watershed. Paddy and Water Environment 7:163-175.

https://doi.org/10.1007/s10333-009-0160-3.

Mattingly JC, Pillai NS, Stuart AM (2012) Diffusion Limits of the Random Walk Metropolis Algorithm in High Dimensions. The Annals of Applied Probability 22:881-930. https://doi.org/10.1214/10-AAP754.

Miao DY, Huang WW, Li YP, Yang ZF (2014) Planning Water Resources Systems under Uncertainty Using an Interval-Fuzzy De Novo Programming Method. Journal of Environmental Informatics 24:11-23. https://doi.org/10.3808/jei.201400277. 
Nguyen NP, Shortle JS, Reed PM, Nguyen TT (2013) Water quality trading with asymmetric information, uncertainty and transaction costs: a stochastic agent-based simulation. Resource and Energy Economics 35:60-90. https://doi.org/10.1016/j.reseneeco.2012.09.002.

Ning HL, Zuo H, Tian JN (2017) Research of phased comprehensive control of Moshui river basin. Environmental Protection Science. https://doi.org/10.16803/j.cnki.issn.1004-6216.2017.04.008.

Pastori M, Bidoglio G, Udias A, Bouraoui F (2017) A multi-objective approach to evaluate the economic and environmental impacts of alternative water and nutrient management strategies in Africa. Journal of Environment Informatics 29:16-28. https://doi.org/10.3808/jei.201500313.

Piao MJ, Li YP, Huang GH (2014) Development of a stochastic simulation-optimization model for planning electric power systems - A case study of Shanghai, China. Energy Conversion and Management 86:111-124. https://doi.org/10.1016/j.enconman.2014.05.011.

Qiao ZJ, Zuo LL, RenYP (2012) Study on Current Situation and Countermeasures of Water Pollution in Moshui River, Jimo. Shandong Water Resources 45-46. (In chinese)

Rajabi MM, Ataie-Ashtiani B, Janssen H (2015) Efficiency enhancement of optimized Latin hypercube sampling strategies: application to Monte Carlo uncertainty analysis and meta-modeling. Advances in Water Resources 76:127-139. https://doi.org/10.1016/j.advwatres.2014///.12.008.

Raje D, Krishnan R (2012) Bayesian parameter uncertainty modeling in a macroscale hydrologic model and its impact on Indian river basin hydrology under climate change. Water Resources Research 48:W08522. https://doi.org/10.1029/2011WR011123. 
Rong QQ, Cai YP, Chen B, Yue WC, Yin XN, Tan Q (2017) An enhanced export coefficient based optimization model for supporting agricultural nonpoint source pollution mitigation under uncertainty. Science of The Total Environment 580:1351-1362.

https://doi.org/10.1016/j.scitotenv.2016.12.099.

Shang X, Wang X, Zhang DL, Chen WD, Chen XC, Kong HN (2012) An improved SWAT-based computational framework for identifying critical source areas for agricultural pollution at the lake basin scale. Ecological Modeling 226:1-10. https://doi.org/10.1016/j.ecolmodel.2011.11.030.

Shen ZY, Chen L, Liao Q (2015) Effect of rainfall measurement errors on nonpoint-source pollution model uncertainty. Journal of Environment Informatics 26:14-26. https://doi.org/10.3808/jei.201400271.

Sheng ML, Liu JZ, Zhu AX, Rossiter DG, Liu HR, Liu ZC, Zhu LM (2019) Comparison of GLUE and DREAM for the estimation of cultivar parameters in the APSIM-maize model. Agricultural and Forest Meteorology 278:107659. https://doi.org/10.1016/j.agrformet.2019.107659.

Shrestha NK, Wang J (2020) Water quality management of a cold climate region watershed in changing climate. Journal of environment informatics 35:56-80. https://doi.org/10.3808/jei.201900407.

Sith R, Watanabe A, Nakamura T, Yamamoto T, Nadaoka K (2019) Assessment of water quality and evaluation of best management practices in a small agricultural watershed adjacent to Coral Reef area in Japan. Agricultural Water Management 213:659-673. https://doi.org/10.1016/j.agwat.2018.11.014. 
Sun JW, Zhang XL, Zhang B (2016) Construction and Application of Information Management System about the Groundwater Source Field of Dagu River. Gound Water 38:57-60.

Vrugt JA (2016) Markov chain Monte Carlo simulation using the DREAM software package: theory, concepts, and MATLAB implementation. Environmental Modelling and Software 75:273-316. https://doi.org/10.1016/j.envsoft.2015.08.013.

Vrugt JA, Beven KJ (2018) Embracing equifinality with efficiency: Limits of Acceptability sampling using the DREAM (LOA) algorithm. Journal of Hydrology 559:954-971. https://doi.org/10.1016/j.jhydrol.2018.02.026.

Vrugt JA, Braak CT, Diks CGH, Robinson BA, Hyman JM, Higdon D (2009) Accelerating markov chain monte carlo simulation by differential evolution with self-adaptive randomized subspace sampling. International Journal of Nonlinear Sciences and Numerical Simulation 10:273-290. https://doi.org/10.1515/IJNSNS.2009.10.3.273.

Wang L (2020) A two-stage stochastic programming framework for evacuation planning in disaster responses. Computers \& Industrial Engineering 145:106458. https://doi.org/10.1016/j.cie.2020.106458.

Wang SQ, Pang J (2019) Research on the Impact of Cross-Regional Emissions Trading on Green Economy. Ecological Economy 35:174-179+196. https://doi.org/CNKI:SUN:STJJ.0.2019-02-031.

Wu W, Ren JC, Zhou XD, Wang JW, Guo MJ (2020) Identification of source information for sudden water pollution incidents in rivers and lakes based on variable-fidelity surrogate-DREAM optimization. Environmental Modelling and Software 133. https://doi.org/10.1016/j.envsoft.2020.104811. 
Xie YL, Li YP, Huang GH, Li YF, Chen LR (2011) An inexact chance-constrained programming model for water quality management in Binhai New Area of Tianjin, China. Science of The Total Environment 409:1757-1773. https://doi.org/10.1016/j.scitotenv.2011.01.036.

Xu J (2004) Application of QUAL2E model for prediction of water quality of Dagu River. Rural Eco-environment.

Xu Y, Qin XS (2010) Agricultural effluent control under uncertainty: An inexact double-sided fuzzy chance-constrained model. Advances in Water Resources 33:997-1014. https://doi.org/10.1016/j.advwatres.2010.06.002.

Zeng XT, Li YP, Huang GH, Liu J (2015) A two-stage interval-stochastic water trading model for allocating water resources of Kaidu-Kongque River in northwestern China. Journal of Hydroinformatics 17:551-569. https://doi.org/10.2166/hydro.2015.090.

Zeng XT, Li YP, Huang GH, Liu J (2016) Modeling water trading under uncertainty for supporting water resources management in an arid region. Journal of Water Resources Planning and Management 142:04015058. https://doi.org/10.1061/(ASCE)WR.1943-5452.0000593.

Zhai Y, Zhao RF, Li YB, Li Y, Meng FD, Wang TN (2020) Stochastic inversion method for dynamic constitutive model of rock materials based on improved DREAM. International Journal of Impact Engineering 147:103739. https://doi.org/10.1016/j.ijimpeng.2020.103739.

Zhang C, Guo S, Zhang F, Engel BA, Guo P (2019) Towards sustainable water resources planning and pollution control: inexact joint-probabilistic double-sided stochastic chance-constrained programming model. Science of the Total Environment 657:73-86. https://doi.org/10.1016/j.scitotenv.2018.11.463. 
Zhang G, Zhao, YG (2008) SOTER database for China, scale 1:1 million. Institute of Soil Science, Chinese Institute of Soil Science, Nanjing.

Zhang JL, Li YP, Zeng XT, Huang GH, Li Y, Zhu Y, Kong FL, Xi M, Liu J (2019) Effluent trading planning and its application in water quality management: A factor-interaction perspective. Environmental Research 168:286-305.

https://doi.org/10.1016/j.envres.2018.09.029.

Zhang XD, Huang GH, Nie XH (2009) Optimal decision schemes for agricultural water quality management planning with imprecise objective. Agricultural Water Management 96:1723-1731. https://doi.org/10.1016/j.agwat.2009.07.011.

Zhu, Y., Li, Y.P., Huang, G.H., Fan, Y.R., Nie, S (2015) A dynamic model to optimize municipal electric power systems by considering carbon emission trading under uncertainty. Energy 88:636-649. https://doi.org/10.1016/j.energy.2015.05.106.

Zolfagharipoor MA, Ahmadi A (2017) Effluent trading in river systems through stochastic decision-making process: a case study. Environmental Science and Pollution Research 24:20655-20672. https://doi.org/10.1007/s11356-017-9720-z. 


\section{List of Table Captions}

Table 1 Sensitive parameters of SWAT model for TP and $\mathrm{NH}_{3}-\mathrm{N}$

Table $2 \mathrm{TP}$ and $\mathrm{NH}_{3}-\mathrm{N}$ loading distribution and the associated probabilities (unit ton)

Table 3 The detailed trading process for TP under Case 1 when $p=0.01$ and $w=2$

Table 4 The detailed trading process for $\mathrm{NH}_{3}-\mathrm{N}$ under Case 1 when $p=0.01$ and $w=2$

Table 5 The detailed trading process for TP under Case 2 when $p=0.01$ and $w=2$

Table 6 The detailed trading process for $\mathrm{NH}_{3}-\mathrm{N}$ under Case 2 when $p=0.01$ and $w=2$

Table 7 Total excess $\mathrm{TP}$ and $\mathrm{NH}_{3}-\mathrm{N}$ emissions in agriculture under three cases (ton)

Table 8 Total excess TP and $\mathrm{NH}_{3}-\mathrm{N}$ emissions under three cases (ton) 
Table 1 Sensitive parameters of SWAT model for TP and $\mathrm{NH}_{3}-\mathrm{N}$

\begin{tabular}{|c|c|c|c|c|c|}
\hline & \multirow{2}{*}{ Parameter } & \multirow{2}{*}{ Description } & \multicolumn{2}{|c|}{ Limit value range } & \multirow{2}{*}{ Units } \\
\hline & & & Min & $\operatorname{Max}$ & \\
\hline \multirow{10}{*}{ ТP } & CH_N (2) & Manning's " $n$ " value for the main channel & 0 & 0.3 & \\
\hline & ALPHA_BF & Baseflow recession factor & 0.001 & 0.056 & \\
\hline & USLE_P & USLE equation support practice factor & 0 & 1 & \\
\hline & REVAPMN & Threshold depth of water in the shallow aquifer for "revap" to occur & 0 & 500 & $\mathrm{~mm}$ \\
\hline & CH_K2 & Effective hydraulic conductivity in main channel alluvium & 0 & 500 & $\mathrm{~mm} / \mathrm{hr}$ \\
\hline & GW_REVAP & Groundwater revap coefficient & 0.02 & 0.2 & \\
\hline & LAT_TTIME & Lateral flow travel time & 0 & 180 & \\
\hline & GWSOLP & Concentration of soluble phosphorus in groundwater contribution to streamflow from subbasin & 0 & 1000 & $\mathrm{mg} \mathrm{P} / 1$ \\
\hline & SOL_AWC & Available water capacity of the soil layer & 0 & 1 & $\mathrm{~mm} / \mathrm{mm}$ soil \\
\hline & $\mathrm{CN} 2$ & SCS runoff curve number for moisture condition II & 35 & 98 & \\
\hline \multirow{9}{*}{$\mathrm{NH}_{3}-\mathrm{N}$} & SOL_AWC & Available water capacity of the soil layer & 0 & 1 & $\mathrm{~mm} / \mathrm{mm}$ soil \\
\hline & SURLAG & Surface runoff lag coefficient & 0.05 & 24 & \\
\hline & SHALLST_N & Concentration of nitrate in groundwater contribution to streamflow from subbasin & 0 & 1000 & $\mathrm{mg} \mathrm{N} / 1$ \\
\hline & GWQMN & Treshold depth of water in the shallow aquifer required for return flow to occur & 0 & 5000 & $\mathrm{~mm}$ \\
\hline & $\mathrm{BC} 2$ & Rate constant for biological oxidation of $\mathrm{NO} 2$ to $\mathrm{NO} 3$ in the reach at $20^{\circ} \mathrm{C}$. & 0.2 & 2 & 1/day \\
\hline & REVAPMN & Threshold depth of water in the shallow aquifer for "revap" to occur & 0 & 500 & $\mathrm{~mm}$ \\
\hline & CH_K2 & Effective hydraulic conductivity in main channel alluvium & 0 & 500 & $\mathrm{~mm} / \mathrm{hr}$ \\
\hline & HRU_SLP & Average slope steepness & 0 & 0.6 & \\
\hline & N_UPDIS & Nitrogen uptake distribution parameter & 0 & 100 & \\
\hline
\end{tabular}


Table $2 \mathrm{TP}$ and $\mathrm{NH}_{3}-\mathrm{N}$ loading distribution and the associated probabilities (unit ton)

\begin{tabular}{llll}
\hline TP loading level & Low $(k=1)$ & Medium $(k=2)$ & High $(k=3)$ \\
\hline Probability & 0.42 & 0.46 & 0.12 \\
Lower-bound loading & 109.4 & 313.4 & 570.2 \\
Probability & 0.42 & 0.46 & 0.12 \\
Upper-bound loading & 419.9 & 1083.0 & 1751.4 \\
\hline $\mathrm{NH}_{3}$-N loading level & Low $(s=1)$ & Medium $(s=2)$ & High $(s=3)$ \\
\hline Probability & 0.36 & 0.53 & 0.11 \\
Lower-bound loading & 112.3 & 342.8 & 662.7 \\
Probability & 0.36 & 0.53 & 0.11 \\
Upper-bound loading & 638.5 & 1598.8 & 2457.0 \\
\hline
\end{tabular}


Table 3 The detailed trading process for TP under Case 1 when $p=0.01$ and $w=1$

\begin{tabular}{|c|c|c|c|c|c|c|c|c|c|}
\hline Seller/purchaser & $i=1$ & $i=2$ & $i=3$ & $i=4$ & $i=5$ & $n=1$ & $n=2$ & $n=3$ & $p=1$ \\
\hline$i=1$ & $0 / 0$ & $0 / 0$ & $0 / 0$ & $35.43 / 14.82$ & $0 / 0$ & $0 / 0$ & $0 / 0$ & $1.32 / 1.08$ & $0 / 0$ \\
\hline$i=2$ & $0 / 0$ & $0 / 0$ & $0 / 0$ & $0 / 0$ & $0 / 0$ & $0 / 0$ & $0 / 0$ & $0 / 0$ & $0 / 0$ \\
\hline$i=3$ & $0 / 0$ & $0 / 0$ & $0 / 0$ & $25.39 / 19.68$ & $0 / 0$ & $0 / 0$ & $0 / 0$ & $0 / 0$ & $0 / 0$ \\
\hline$i=4$ & $0 / 0$ & $3.32 / 3.32$ & $0 / 0$ & $0 / 0$ & $0 / 0$ & $0 / 0$ & $0 / 0$ & $0 / 0$ & $0 / 0$ \\
\hline$i=5$ & $0 / 0$ & $0 / 0$ & $0 / 0$ & $0 / 0$ & $0 / 0$ & $0 / 0$ & $0 / 0$ & $0 / 0$ & $0 / 0$ \\
\hline$n=1$ & $0 / 0$ & $0 / 0$ & $0 / 0$ & $0 / 0$ & $0 / 0$ & $0 / 0$ & $0 / 0$ & $0 / 0$ & $1.18 / 1.13$ \\
\hline$n=2$ & $0 / 0$ & $0 / 0$ & $19.05 / 18.45$ & $0 / 0$ & $0 / 0$ & $0 / 0$ & $0 / 0$ & $0 / 0$ & $0 / 0$ \\
\hline$n=3$ & $0 / 0$ & $0 / 0$ & $0 / 0$ & $0 / 0$ & $0 / 0$ & $0 / 0$ & $3.42 / 2.63$ & $0 / 0$ & $0 / 0$ \\
\hline$p=1$ & $0 / 0$ & $0 / 0$ & $0 / 0$ & $0 / 0$ & $0 / 0$ & $3.44 / 3.44$ & $0 / 0$ & $0 / 0$ & $0 / 0$ \\
\hline$p=2$ & $3.34 / 3.34$ & $7.89 / 6.16$ & $0 / 0$ & $7.89 / 5.48$ & $0 / 0$ & $0 / 0$ & $0 / 0$ & $0 / 0$ & $0 / 0$ \\
\hline$p=3$ & $0 / 0$ & $0.52 / 0.31$ & $0 / 0$ & 0 & $0 / 0$ & $0 / 0$ & $4.47 / 3.6$ & $0 / 0$ & $0 / 0$ \\
\hline$m=1$ & l & l & l & l & $0 / 0$ & l & l & I & l \\
\hline$m=2$ & l & l & l & l & $0.2 / 0.2$ & I & l & I & l \\
\hline$m=3$ & l & l & l & l & $0 / 0$ & l & l & I & l \\
\hline$m=4$ & 1 & 1 & I & I & $0.01 / 0$ & l & l & 1 & l \\
\hline$m=5$ & 1 & 1 & I & I & $0 / 0$ & l & l & 1 & l \\
\hline$m=6$ & 1 & 1 & I & I & $0 / 0$ & l & l & 1 & l \\
\hline$m=7$ & 1 & 1 & 1 & 1 & $0.82 / 0.82$ & 1 & 1 & 1 & 1 \\
\hline Seller/purchaser & $p=2$ & $p=3$ & $m=1$ & $m=2$ & $m=3$ & $m=4$ & $m=5$ & $m=6$ & $m=7$ \\
\hline$i=1$ & $0 / 0$ & $0 / 0$ & l & l & 1 & l & I & l & l \\
\hline$i=2$ & $0 / 0$ & $0 / 0$ & l & l & I & l & l & 1 & I \\
\hline$i=3$ & $0 / 0$ & $0 / 0$ & l & 1 & I & l & l & 1 & I \\
\hline$i=4$ & $34.70 / 34.70$ & $0 / 0$ & l & 1 & I & l & l & 1 & I \\
\hline$i=5$ & $0 / 0$ & $0 / 0$ & $0 / 0$ & $0 / 0$ & $0 / 0$ & $0 / 0$ & $0.08 / 0.03$ & $0 / 0$ & $0 / 0$ \\
\hline$n=1$ & $0 / 0$ & $2.2 / 1.28$ & l & l & l & I & I & 1 & I \\
\hline$n=2$ & $0 / 0$ & $0 / 0$ & l & l & l & l & l & I & I \\
\hline$n=3$ & $0 / 0$ & $0 / 0$ & l & 1 & 1 & l & l & 1 & l \\
\hline$p=1$ & $0 / 0$ & $0 / 0$ & l & l & l & I & l & I & I \\
\hline$p=2$ & $0 / 0$ & $0 / 0$ & l & l & l & l & l & I & I \\
\hline$p=3$ & $0 / 0$ & $0 / 0$ & l & l & l & l & l & I & I \\
\hline$m=1$ & I & 1 & $0 / 0$ & $0.2 / 0.2$ & $0 / 0$ & $0 / 0$ & $0.02 / 0.01$ & $0 / 0$ & $0 / 0$ \\
\hline$m=2$ & l & l & $0 / 0$ & $0 / 0$ & $0 / 0$ & $0.01 / 0.01$ & $0.02 / 0.01$ & $0 / 0$ & $0.38 / 0.38$ \\
\hline$m=3$ & I & 1 & $0 / 0$ & $0 / 0$ & $0 / 0$ & $0 / 0$ & $0 / 0$ & $0 / 0$ & $0 / 0$ \\
\hline$m=4$ & I & 1 & $0 / 0$ & $0 / 0$ & $0 / 0$ & $0 / 0$ & $0 / 0$ & $0 / 0$ & $0 / 0$ \\
\hline$m=5$ & I & I & $0 / 0$ & $0.15 / 0.15$ & $0 / 0$ & $0 / 0$ & $0 / 0$ & $0 / 0$ & $0 / 0$ \\
\hline$m=6$ & I & I & $0 / 0$ & $0 / 0$ & $0 / 0$ & $0 / 0$ & $0 / 0$ & $0 / 0$ & $0 / 0$ \\
\hline$m=7$ & 1 & 1 & $0.13 / 0.13$ & $0 / 0$ & $0 / 0$ & $0 / 0$ & $0.02 / 0.01$ & $0 / 0$ & $0 / 0$ \\
\hline
\end{tabular}


Table 4 The detailed trading process for $\mathrm{NH}_{3}-\mathrm{N}$ under Case 1 when $p=0.01$ and $w=1$

\begin{tabular}{|c|c|c|c|c|c|c|c|c|c|}
\hline Seller/purchaser & $i=1$ & $i=2$ & $i=3$ & $i=4$ & $i=5$ & $n=1$ & $n=2$ & $n=3$ & $p=1$ \\
\hline$i=1$ & $0 / 0$ & $440.76 / 209.89$ & $0 / 0$ & $0 / 0$ & $0 / 0$ & $0 / 0$ & $0 / 0$ & $0 / 0$ & $0 / 0$ \\
\hline$i=2$ & $0 / 0$ & $0 / 0$ & $0 / 0$ & $177.16 / 168.72$ & $0 / 0$ & $228 / 228$ & $0 / 0$ & $0 / 0$ & $0 / 0$ \\
\hline$i=3$ & $0 / 0$ & $0 / 0$ & $0 / 0$ & $177.16 / 125.64$ & $0 / 0$ & $0 / 0$ & $0 / 0$ & $0 / 0$ & $45.07 / 45.07$ \\
\hline$i=4$ & $0 / 0$ & $0 / 0$ & $0 / 0$ & $0 / 0$ & $0 / 0$ & $177.16 / 177.16$ & $49.15 / 49.15$ & $0 / 0$ & $0 / 0$ \\
\hline$i=5$ & $0 / 0$ & $0 / 0$ & $0 / 0$ & $0 / 0$ & $0 / 0$ & $0 / 0$ & $0 / 0$ & $0 / 0$ & $0 / 0$ \\
\hline$n=1$ & $0 / 0$ & $154.6 / 54.82$ & $154.6 / 79.7$ & $32.28 / 11.17$ & $0 / 0$ & $0 / 0$ & $120.04 / 57.16$ & $0 / 0$ & $0 / 0$ \\
\hline$n=2$ & $0 / 0$ & $0 / 0$ & $0 / 0$ & $0 / 0$ & $0 / 0$ & $0 / 0$ & $0 / 0$ & $0 / 0$ & $0 / 0$ \\
\hline$n=3$ & $8.40 / 8.40$ & $0 / 0$ & $21.83 / 16.29$ & $0 / 0$ & $0 / 0$ & $0 / 0$ & $0 / 0$ & $0 / 0$ & $0 / 0$ \\
\hline$p=1$ & $0 / 0$ & $0 / 0$ & $0 / 0$ & $0 / 0$ & $0 / 0$ & $0 / 0$ & $0 / 0$ & $0 / 0$ & $39.43 / 18.78$ \\
\hline$p=2$ & $0 / 0$ & $39.43 / 30.8$ & $0 / 0$ & $10.43 / 7.73$ & $0 / 0$ & $39.43 / 39.43$ & $0 / 0$ & $39.43 / 39.43$ & $0 / 0$ \\
\hline$p=3$ & $34.12 / 34.12$ & $34.12 / 18.95$ & $34.12 / 26.66$ & $34.12 / 19.06$ & $0 / 0$ & $34.12 / 34.12$ & $0 / 0$ & $0 / 0$ & $0 / 0$ \\
\hline$m=1$ & / & l & / & / & $2.3 / 2.3$ & / & / & / & / \\
\hline$m=2$ & l & l & l & I & $3.46 / 3.46$ & / & / & / & / \\
\hline$m=3$ & / & l & / & / & $0.01 / 0.01$ & / & / & / & / \\
\hline$m=4$ & l & / & / & / & $0.14 / 0.14$ & / & / & / & / \\
\hline$m=5$ & / & l & / & / & $0.36 / 0.36$ & / & / & / & / \\
\hline$m=6$ & l & / & / & / & $0.06 / 0.06$ & / & / & / & / \\
\hline$m=7$ & / & l & / & / & $29.1 / 29.1$ & / & / & / & / \\
\hline Seller/purchaser & $p=2$ & $p=3$ & $m=1$ & $m=2$ & $m=3$ & $m=4$ & $m=5$ & $m=6$ & $m=7$ \\
\hline$i=1$ & $0 / 0$ & $0 / 0$ & / & / & / & / & / & l & / \\
\hline$i=2$ & $0 / 0$ & $0 / 0$ & / & / & / & / & / & / & / \\
\hline$i=3$ & $0 / 0$ & $0 / 0$ & / & / & / & / & l & / & / \\
\hline$i=4$ & $0 / 0$ & $174.39 / 174.39$ & / & I & / & I & / & I & / \\
\hline$i=5$ & $0 / 0$ & $0 / 0$ & $42.67 / 34.14$ & $0 / 0$ & $0 / 0$ & $0 / 0$ & $0 / 0$ & $0 / 0$ & $0 / 0$ \\
\hline$n=1$ & $0 / 0$ & $0 / 0$ & / & / & / & / & / & / & / \\
\hline$n=2$ & $120.04 / 114.33$ & $0 / 0$ & / & / & / & / & / & / & / \\
\hline$n=3$ & $0 / 0$ & $0 / 0$ & / & / & / & / & I & I & / \\
\hline$p=1$ & $0 / 0$ & $0 / 0$ & / & / & / & / & I & / & / \\
\hline$p=2$ & $0 / 0$ & $0 / 0$ & / & / & l & / & / & / & / \\
\hline$p=3$ & $0 / 0$ & $0 / 0$ & I & I & I & / & l & / & / \\
\hline$m=1$ & / & l & $0 / 0$ & $3.46 / 3.46$ & $0.01 / 0.01$ & $0.14 / 0.14$ & $0.36 / 0.18$ & $0 / 0$ & $29.1 / 29.1$ \\
\hline$m=2$ & / & I & $0 / 0$ & $0 / 0$ & $0.01 / 0.01$ & $0 / 0$ & $0.36 / 0.18$ & $0.06 / 0.06$ & $0 / 0$ \\
\hline$m=3$ & I & l & $0 / 0$ & $0 / 0$ & $0 / 0$ & $0 / 0$ & $0 / 0$ & $0 / 0$ & $0.02 / 0.02$ \\
\hline$m=4$ & I & l & $0 / 0$ & $0 / 0$ & $0.01 / 0.01$ & $0 / 0$ & $0 / 0$ & $0.06 / 0.06$ & $0 / 0$ \\
\hline$m=5$ & l & l & $0 / 0$ & $0 / 0$ & $0 / 0$ & $0.08 / 0.08$ & $0 / 0$ & $0 / 0$ & $0.07 / 0.07$ \\
\hline$m=6$ & / & l & $0 / 0$ & $0 / 0$ & $0.01 / 0.01$ & $0 / 0$ & $0 / 0$ & $0 / 0$ & $0.05 / 0.05$ \\
\hline$m=7$ & 1 & 1 & $0 / 0$ & $0.81 / 0.81$ & $0 / 0$ & $0 / 0$ & $0.36 / 0.18$ & $0 / 0$ & $0 / 0$ \\
\hline
\end{tabular}


Table 5 The detailed trading process for TP under Case 2 when $p=0.01$ and $w=1$

\begin{tabular}{|c|c|c|c|c|c|c|c|c|c|}
\hline Seller/purchaser & $i=1$ & $i=2$ & $i=3$ & $i=4$ & $i=5$ & $n=1$ & $n=2$ & $n=3$ & $p=1$ \\
\hline$i=1$ & $0 / 0$ & $0 / 0$ & $0 / 0$ & $0 / 0$ & $0.41 / 0.31$ & $0 / 0$ & $0 / 0$ & $0 / 0$ & $4.56 / 4.56$ \\
\hline$i=2$ & $0 / 0$ & $0 / 0$ & $4.12 / 4.12$ & $0 / 0$ & $0 / 0$ & $0 / 0$ & $0 / 0$ & $0 / 0$ & $0 / 0$ \\
\hline$i=3$ & $0 / 0$ & $0 / 0$ & $0 / 0$ & $0 / 0$ & $23.73 / 23.73$ & $0 / 0$ & $0 / 0$ & $0 / 0$ & $0 / 0$ \\
\hline$i=4$ & $0 / 0$ & $0 / 0$ & $0 / 0$ & $0 / 0$ & $0 / 0$ & $0 / 0$ & $0 / 0$ & $0 / 0$ & $0 / 0$ \\
\hline$i=5$ & $0 / 0$ & $0 / 0$ & $0 / 0$ & $0.88 / 0.88$ & $0 / 0$ & $0 / 0$ & $0 / 0$ & $0 / 0$ & $0 / 0$ \\
\hline$n=1$ & $0 / 0$ & $0 / 0$ & $0 / 0$ & $0 / 0$ & $0 / 0$ & $0 / 0$ & $0 / 0$ & $0 / 0$ & $0 / 0$ \\
\hline$n=2$ & $0 / 0$ & $0 / 0$ & $0 / 0$ & $0 / 0$ & $0 / 0$ & $0 / 0$ & $0 / 0$ & $0 / 0$ & $0 / 0$ \\
\hline$n=3$ & $0 / 0$ & $0 / 0$ & $2.36 / 1.76$ & $0 / 0$ & $0 / 0$ & $0 / 0$ & $0 / 0$ & $0 / 0$ & $0 / 0$ \\
\hline$p=1$ & $0 / 0$ & $0 / 0$ & $0 / 0$ & $0 / 0$ & $0 / 0$ & $3.44 / 3.44$ & $0 / 0$ & $0 / 0$ & $0 / 0$ \\
\hline$p=2$ & $0 / 0$ & $7.89 / 6.16$ & $7.89 / 7.89$ & $0 / 0$ & $0 / 0$ & $0 / 0$ & $2.67 / 2.67$ & $0 / 0$ & $0 / 0$ \\
\hline$p=3$ & $0 / 0$ & $0 / 0$ & $2.2 / 1.72$ & $0 / 0$ & $0 / 0$ & $0 / 0$ & $0 / 0$ & $0 / 0$ & $0 / 0$ \\
\hline$m=1$ & $0 / 0$ & $0.13 / 0.13$ & $0.13 / 0.13$ & $0.13 / 0.13$ & $0.13 / 0.13$ & $0 / 0$ & $0 / 0$ & $0 / 0$ & $0 / 0$ \\
\hline$m=2$ & $0 / 0$ & $0.2 / 0.2$ & $0.2 / 0.2$ & $0.2 / 0.2$ & $0.2 / 0.2$ & $0 / 0$ & $0 / 0$ & $0 / 0$ & $0 / 0$ \\
\hline$m=3$ & $0 / 0$ & $0 / 0$ & $0 / 0$ & $0 / 0$ & $0 / 0$ & $0 / 0$ & $0 / 0$ & $0 / 0$ & $0 / 0$ \\
\hline$m=4$ & $0 / 0$ & $0.01 / 0.01$ & $0.01 / 0.01$ & $0.01 / 0.01$ & $0.01 / 0.01$ & $0 / 0$ & $0 / 0$ & $0.01 / 0.01$ & $0 / 0$ \\
\hline$m=5$ & $0 / 0$ & $0 / 0$ & $0 / 0$ & $0 / 0$ & $0 / 0$ & $0 / 0$ & $0 / 0$ & $0 / 0$ & $0 / 0$ \\
\hline$m=6$ & $0 / 0$ & $0 / 0$ & $0 / 0$ & $0 / 0$ & $0 / 0$ & $0 / 0$ & $0 / 0$ & $0 / 0$ & $0 / 0$ \\
\hline$m=7$ & $0 / 0$ & $0.97 / 0.97$ & $0.97 / 0.97$ & $0.97 / 0.97$ & $0.97 / 0.97$ & $0 / 0$ & $0 / 0$ & $0 / 0$ & $0 / 0$ \\
\hline Seller/purchaser & $p=2$ & $p=3$ & $m=1$ & $m=2$ & $m=3$ & $m=4$ & $m=5$ & $m=6$ & $m=7$ \\
\hline$i=1$ & $0 / 0$ & $0 / 0$ & $0 / 0$ & $0 / 0$ & $0 / 0$ & $0.03 / 0.03$ & $0 / 0$ & $0 / 0$ & $0 / 0$ \\
\hline$i=2$ & $0 / 0$ & $0 / 0$ & $0 / 0$ & $0 / 0$ & $0 / 0$ & $0 / 0$ & $0 / 0$ & $0 / 0$ & $0 / 0$ \\
\hline$i=3$ & $0 / 0$ & $0 / 0$ & $0 / 0$ & $0 / 0$ & $0 / 0$ & $0 / 0$ & $0 / 0$ & $0 / 0$ & $0 / 0$ \\
\hline$i=4$ & $0 / 0$ & $0 / 0$ & $0 / 0$ & $0 / 0$ & $0 / 0$ & $0 / 0$ & $0 / 0$ & $0 / 0$ & $0 / 0$ \\
\hline$i=5$ & $26.66 / 26.66$ & $0 / 0$ & $0 / 0$ & $0 / 0$ & $0 / 0$ & $0 / 0$ & $0 / 0$ & $0 / 0$ & $0 / 0$ \\
\hline$n=1$ & $0 / 0$ & $0 / 0$ & $0 / 0$ & $0 / 0$ & $0 / 0$ & $0 / 0$ & $0 / 0$ & $0 / 0$ & $0 / 0$ \\
\hline$n=2$ & $0 / 0$ & $0 / 0$ & $0 / 0$ & $0 / 0$ & $0 / 0$ & $0 / 0$ & $0 / 0$ & $0 / 0$ & $0 / 0$ \\
\hline$n=3$ & $0 / 0$ & $0 / 0$ & $0 / 0$ & $0 / 0$ & $0 / 0$ & $0 / 0$ & $0 / 0$ & $0 / 0$ & $0 / 0$ \\
\hline$p=1$ & $0 / 0$ & $0 / 0$ & $0 / 0$ & $0 / 0$ & $0 / 0$ & $0 / 0$ & $0 / 0$ & $0 / 0$ & $0 / 0$ \\
\hline$p=2$ & $0 / 0$ & $2.62 / 2.62$ & $0 / 0$ & $0.08 / 0.08$ & $0 / 0$ & $0 / 0$ & $0.01 / 0.01$ & $0 / 0$ & $4.64 / 4.64$ \\
\hline$p=3$ & $0 / 0$ & $0 / 0$ & $0 / 0$ & $0 / 0$ & $0 / 0$ & $0 / 0$ & $0 / 0$ & $0 / 0$ & $0 / 0$ \\
\hline$m=1$ & $0 / 0$ & $0.13 / 0.13$ & $0 / 0$ & $0 / 0$ & $0 / 0$ & $0 / 0$ & $0 / 0$ & $0 / 0$ & $0.13 / 0.13$ \\
\hline$m=2$ & $0 / 0$ & $0.2 / 0.2$ & $0.20 / 0.20$ & $0 / 0$ & $0 / 0$ & $0 / 0$ & $0 / 0$ & $0.02 / 0.02$ & $0 / 0$ \\
\hline$m=3$ & $0 / 0$ & $0 / 0$ & $0 / 0$ & $0 / 0$ & $0 / 0$ & $0 / 0$ & $0 / 0$ & $0 / 0$ & $0 / 0$ \\
\hline$m=4$ & $0 / 0$ & $0.01 / 0.01$ & $0 / 0$ & $0 / 0$ & $0 / 0$ & $0 / 0$ & $0 / 0$ & $0 / 0$ & $0 / 0$ \\
\hline$m=5$ & $0 / 0$ & $0 / 0$ & $0 / 0$ & $0.02 / 0.02$ & $0 / 0$ & $0 / 0$ & $0 / 0$ & $0 / 0$ & $0 / 0$ \\
\hline$m=6$ & $0 / 0$ & $0 / 0$ & $0 / 0$ & $0 / 0$ & $0 / 0$ & $0 / 0$ & $0 / 0$ & $0 / 0$ & $0 / 0$ \\
\hline$m=7$ & $0 / 0$ & $0 / 0$ & $0.51 / 0.51$ & $0.97 / 0.97$ & $0 / 0$ & $0 / 0$ & $0 / 0$ & $0 / 0$ & $0 / 0$ \\
\hline
\end{tabular}


Table 6 The detailed trading process for $\mathrm{NH}_{3}-\mathrm{N}$ under Case 2 when $p=0.01$ and $w=1$

\begin{tabular}{|c|c|c|c|c|c|c|c|c|c|}
\hline Seller/purchaser & $i=1$ & $i=2$ & $i=3$ & $i=4$ & $i=5$ & $n=1$ & $n=2$ & $n=3$ & $p=1$ \\
\hline$i=1$ & $0 / 0$ & $296.19 / 141.04$ & $119.99 / 82.18$ & $0 / 0$ & $0 / 0$ & $0 / 0$ & $0 / 0$ & $0 / 0$ & $0 / 0$ \\
\hline$i=2$ & $307.09 / 307.09$ & $0 / 0$ & $0 / 0$ & $0 / 0$ & $0 / 0$ & $0 / 0$ & $0 / 0$ & $0 / 0$ & $0 / 0$ \\
\hline$i=3$ & $0 / 0$ & $0 / 0$ & $0 / 0$ & $0 / 0$ & $381.4 / 381.4$ & $0 / 0$ & $0 / 0$ & $0 / 0$ & $0 / 0$ \\
\hline$i=4$ & $0 / 0$ & $0 / 0$ & $0 / 0$ & $0 / 0$ & $13.44 / 13.44$ & $0 / 0$ & $0.73 / 0.73$ & $25.31 / 25.31$ & $0 / 0$ \\
\hline$i=5$ & $134.08 / 134.08$ & $0 / 0$ & $0 / 0$ & $0 / 0$ & $0 / 0$ & $0 / 0$ & $134.08 / 134.08$ & $134.08 / 134.08$ & $0 / 0$ \\
\hline$n=1$ & $0 / 0$ & $0 / 0$ & $0 / 0$ & $0 / 0$ & $0 / 0$ & $0 / 0$ & $74.1 / 35.29$ & $0 / 0$ & $0 / 0$ \\
\hline$n=2$ & $0 / 0$ & $94.07 / 70.2$ & $0 / 0$ & $0 / 0$ & $0 / 0$ & $0 / 0$ & $0 / 0$ & $0 / 0$ & $120.04 / 120.04$ \\
\hline$n=3$ & $82.77 / 82.77$ & $0 / 0$ & $82.77 / 61.77$ & $82.77 / 44.03$ & $0 / 0$ & $0 / 0$ & $0 / 0$ & $0 / 0$ & $0 / 0$ \\
\hline$p=1$ & $0 / 0$ & $0 / 0$ & $0 / 0$ & $0 / 0$ & $0 / 0$ & $50.78 / 50.78$ & $50.78 / 25.39$ & $7.68 / 5.56$ & $0 / 0$ \\
\hline$p=2$ & $0 / 0$ & $0 / 0$ & $0 / 0$ & $0 / 0$ & $0 / 0$ & $0 / 0$ & $0 / 0$ & $0 / 0$ & $39.43 / 39.43$ \\
\hline$p=3$ & $0 / 0$ & $0 / 0$ & $0 / 0$ & $0 / 0$ & $0 / 0$ & $0 / 0$ & $0 / 0$ & $34.12 / 34.12$ & $0 / 0$ \\
\hline$m=1$ & $2.30 / 2.30$ & $2.30 / 1.90$ & $2.30 / 2.30$ & $2.30 / 1.90$ & $2.30 / 2.30$ & $2.30 / 2.30$ & $2.30 / 1.79$ & $2.30 / 2.30$ & $2.30 / 2.30$ \\
\hline$m=2$ & $3.46 / 3.46$ & $3.46 / 2.88$ & $0 / 0$ & $3.46 / 2.88$ & $3.46 / 3.46$ & $3.46 / 3.46$ & $3.46 / 2.68$ & $3.46 / 3.46$ & $3.46 / 3.46$ \\
\hline$m=3$ & $0.01 / 0.01$ & $0.01 / 0.01$ & $0.01 / 0.01$ & $0.01 / 0.01$ & $0.01 / 0.01$ & $0.01 / 0.01$ & $0.01 / 0.01$ & $0.01 / 0.01$ & $0.01 / 0.01$ \\
\hline$m=4$ & $0.14 / 0.11$ & $0 / 0$ & $0 / 0$ & $0.14 / 0.11$ & $0 / 0$ & $0 / 0$ & $0.14 / 0.11$ & $0 / 0$ & $0 / 0$ \\
\hline$m=5$ & $0.36 / 0.36$ & $0.36 / 0.36$ & $0.36 / 0.36$ & $0.36 / 0.36$ & $0.36 / 0.36$ & $0.36 / 0.36$ & $0.36 / 0.36$ & $0.36 / 0.36$ & $0.36 / 0.36$ \\
\hline$m=6$ & $0.06 / 0.06$ & $0 / 0$ & $0 / 0$ & $0 / 0$ & $0.06 / 0.06$ & $0 / 0$ & $0 / 0$ & $0 / 0$ & $0.06 / 0.06$ \\
\hline$m=7$ & $0 / 0$ & $0 / 0$ & $0 / 0$ & $0 / 0$ & $0 / 0$ & $0 / 0$ & $0 / 0$ & $0 / 0$ & $0 / 0$ \\
\hline Seller/purchaser & $p=2$ & $p=3$ & $m=1$ & $m=2$ & $m=3$ & $m=4$ & $m=5$ & $m=6$ & $m=7$ \\
\hline$i=1$ & $0 / 0$ & $0 / 0$ & $0 / 0$ & $59.56 / 35.88$ & $0 / 0$ & $0 / 0$ & $0 / 0$ & $0 / 0$ & $0 / 0$ \\
\hline$i=2$ & $0 / 0$ & $0 / 0$ & $0 / 0$ & $0 / 0$ & $0 / 0$ & $0 / 0$ & $0 / 0$ & $0 / 0$ & $0 / 0$ \\
\hline$i=3$ & $0 / 0$ & $0 / 0$ & $0 / 0$ & $0 / 0$ & $0 / 0$ & $0 / 0$ & $0 / 0$ & $0 / 0$ & $0 / 0$ \\
\hline$i=4$ & $0 / 0$ & $0 / 0$ & $0 / 0$ & $0 / 0$ & $0 / 0$ & $0 / 0$ & $0 / 0$ & $0 / 0$ & $0 / 0$ \\
\hline$i=5$ & $14.96 / 14.96$ & $65.84 / 65.84$ & $0 / 0$ & $0 / 0$ & $0 / 0$ & $0 / 0$ & $0 / 0$ & $0 / 0$ & $0 / 0$ \\
\hline$n=1$ & $0 / 0$ & $0 / 0$ & $0 / 0$ & $0 / 0$ & $0 / 0$ & $0 / 0$ & $0 / 0$ & $0 / 0$ & $0 / 0$ \\
\hline$n=2$ & $0 / 0$ & $0 / 0$ & $0 / 0$ & $0 / 0$ & $0 / 0$ & $0 / 0$ & $0 / 0$ & $0 / 0$ & $0 / 0$ \\
\hline$n=3$ & $0 / 0$ & $0 / 0$ & $0 / 0$ & $0 / 0$ & $0 / 0$ & $0 / 0$ & $0 / 0$ & $0 / 0$ & $0 / 0$ \\
\hline$p=1$ & $50.78 / 24.18$ & $0 / 0$ & $0 / 0$ & $0 / 0$ & $0 / 0$ & $0 / 0$ & $0 / 0$ & $0 / 0$ & $0 / 0$ \\
\hline$p=2$ & $0 / 0$ & $0 / 0$ & $0 / 0$ & $0 / 0$ & $0 / 0$ & $0 / 0$ & $0 / 0$ & $0 / 0$ & $0 / 0$ \\
\hline$p=3$ & $0 / 0$ & $0 / 0$ & $34.12 / 30.74$ & $0 / 0$ & $0 / 0$ & $0 / 0$ & $0 / 0$ & $0 / 0$ & $0 / 0$ \\
\hline$m=1$ & $2.30 / 1.71$ & $2.30 / 2.30$ & $0 / 0$ & $2.30 / 2.30$ & $0 / 0$ & $0.53 / 0.53$ & $0 / 0$ & $0 / 0$ & $2.30 / 2.30$ \\
\hline$m=2$ & $3.46 / 2.56$ & $3.46 / 2.56$ & $0 / 0$ & $0 / 0$ & $0 / 0$ & $0 / 0$ & $0 / 0$ & $0.24 / 0.24$ & $3.46 / 3.46$ \\
\hline$m=3$ & $0.01 / 0.01$ & $0.01 / 0.01$ & $0.01 / 0.01$ & $0.01 / 0.01$ & $0 / 0$ & $0.01 / 0.01$ & $0 / 0$ & $0.01 / 0.01$ & $0.01 / 0.01$ \\
\hline$m=4$ & $0 / 0$ & $0 / 0$ & $0 / 0$ & $0.14 / 0.14$ & $0 / 0$ & $0 / 0$ & $0 / 0$ & $0.14 / 0.14$ & $0 / 0$ \\
\hline$m=5$ & $0.36 / 0.36$ & $0.36 / 0.36$ & $0 / 0$ & $0 / 0$ & $0 / 0$ & $0 / 0$ & $0 / 0$ & $0 / 0$ & $0 / 0$ \\
\hline$m=6$ & $0.06 / 0.05$ & $0.06 / 0.06$ & $0 / 0$ & $0 / 0$ & $0 / 0$ & $0.06 / 0.06$ & $0 / 0$ & $0 / 0$ & $0.06 / 0.06$ \\
\hline$m=7$ & $0 / 0$ & $0 / 0$ & $0 / 0$ & $0 / 0$ & $0.13 \backslash 0.13$ & $0 / 0$ & $7.77 / 3.98$ & $0 / 0$ & $0 / 0$ \\
\hline
\end{tabular}


Table 7 Total excess TP and $\mathrm{NH}_{3}-\mathrm{N}$ emissions in agriculture under three cases (ton)

\begin{tabular}{|c|c|c|c|c|c|}
\hline & Case & Probability & $w=1$ & $w=2$ & $w=3$ \\
\hline \multirow{6}{*}{$\mathrm{TP}$} & \multirow{2}{*}{ Case 1} & $p=0.01$ & 0 & 183.54 & 60.78 \\
\hline & & $p=0.1$ & 0 & 183.34 & 60.78 \\
\hline & \multirow{2}{*}{ Case 2} & $p=0.01$ & 0 & 188.49 & 84.14 \\
\hline & & $p=0.1$ & 0 & 187.80 & 73.81 \\
\hline & \multirow{2}{*}{ Case 3} & $p=0.01$ & 0 & 185.35 & 69.87 \\
\hline & & $p=0.1$ & 0 & 185.35 & 69.87 \\
\hline \multirow{6}{*}{$\mathrm{NH}_{3}-\mathrm{N}$} & \multirow{2}{*}{ Case 1} & $p=0.01$ & 0 & 253.83 & 0 \\
\hline & & $p=0.1$ & 0 & 249.27 & 0 \\
\hline & \multirow{2}{*}{ Case 2} & $p=0.01$ & 0 & 273.34 & 0 \\
\hline & & $p=0.1$ & 0 & 268.24 & 0 \\
\hline & \multirow{2}{*}{ Case 3} & $p=0.01$ & 0 & 259.57 & 0 \\
\hline & & $p=0.1$ & 0 & 259.57 & 0 \\
\hline
\end{tabular}


Table 8 Total excess TP and $\mathrm{NH}_{3}-\mathrm{N}$ emissions under three cases (ton)

\begin{tabular}{|c|c|c|c|c|c|}
\hline & Case & Probability & $w=1$ & $w=2$ & $w=3$ \\
\hline \multirow{9}{*}{$\mathrm{TP}$} & \multirow{3}{*}{ Case 1} & $p=0.01$ & 243.16 & 521.48 & 383.11 \\
\hline & & $p=0.05$ & 238.63 & 520.62 & 383.46 \\
\hline & & $p=0.1$ & 238.63 & 520.30 & 380.95 \\
\hline & \multirow{3}{*}{ Case 2} & $p=0.01$ & 231.96 & 521.48 & 380.46 \\
\hline & & $p=0.05$ & 231.96 & 520.62 & 375.40 \\
\hline & & $p=0.1$ & 231.96 & 520.30 & 372.88 \\
\hline & \multirow{3}{*}{ Case 3} & $p=0.01$ & & & \\
\hline & & $p=0.05$ & 240.82 & 519.05 & 370.46 \\
\hline & & $p=0.1$ & & & \\
\hline \multirow{9}{*}{$\mathrm{NH}_{3}-\mathrm{N}$} & \multirow{3}{*}{ Case 1} & $p=0.01$ & 614.11 & 1195.66 & 732.82 \\
\hline & & $p=0.05$ & 614.11 & 1192.36 & 732.82 \\
\hline & & $p=0.1$ & 614.11 & 1190.56 & 732.82 \\
\hline & \multirow{3}{*}{ Case 2} & $p=0.01$ & 614.11 & 1195.66 & 732.82 \\
\hline & & $p=0.05$ & 614.11 & 1192.36 & 732.82 \\
\hline & & $p=0.1$ & 614.11 & 1190.56 & 732.82 \\
\hline & \multirow{3}{*}{ Case 3} & $p=0.01$ & & & \\
\hline & & $p=0.05$ & 627.93 & 1184.46 & 750.31 \\
\hline & & $p=0.1$ & & & \\
\hline
\end{tabular}




\section{List of Figure Captions}

Figure 1 Framework of a Bayesian simulation-based multi-watershed effluent trading designing model (BS-METM)

Figure 2 Location and pollution sources of Daguhe and Moshuihe watersheds

Figure 3 The prediction intervals of TP loading

Figure 4 The prediction intervals of $\mathrm{NH}_{3}-\mathrm{N}$ loading

Figure 5 Cumulated distribution functions of nutrient loadings [(a) low TP loading level for lower bound, (b) medium TP loading level for lower bound, (c) high TP loading level for lower bound, (d) low TP loading level for upper bound, (e) medium TP loading level for upper bound, (f) high TP loading level for upper bound]

Figure 6 Cumulated distribution functions of nutrient loadings [(a) low $\mathrm{NH}_{3}-\mathrm{N}$ loading level for lower bound, (b) medium $\mathrm{NH}_{3}-\mathrm{N}$ loading level for lower bound, (c) high $\mathrm{NH}_{3}-\mathrm{N}$ loading level for lower bound, (d) low $\mathrm{NH}_{3}-\mathrm{N}$ loading level for upper bound, (e) medium $\mathrm{NH}_{3}-\mathrm{N}$ loading level for upper bound, (f) high $\mathrm{NH}_{3}-\mathrm{N}$ loading level for upper bound]

Figure 7 Net system benefits under intra-watershed trading, cross-watershed trading and non-trading cases $[(\mathrm{a}) p=0.01$, (b) $p=0.05$, (c) $p=0.1]$

Figure 8 The total trading amounts under Cases 1 and $2[(\mathrm{a}) \mathrm{TP}, p=0.01$; (b) TP, $p=0.05$, (c) TP, $p=0.1 ;$ (d) NH3-N, $p=0.01 ;$ (e) NH3-N, $p=0.05$, (f) NH3-N, $p=0.1]$

Figure 9 The eliminated trading amounts of tradings under Cases 1 and 2 for TP and NH3-N [(a) TP, $p=0.01$; (b) TP, $p=0.05$; (c) TP, $p=0.1$; (d) NH3-N, $p=0.01$; (e) NH3-N, $p=0.05$; (f) $\mathrm{NH} 3-\mathrm{N}, p=0.1]$

Figure $10 \mathrm{TP}$ and $\mathrm{NH}_{3}-\mathrm{N}$ detailed trading process for agriculture, livestock and poultry industry, 
fishery and company [(a) TP, $p=0.01$; (b) TP, $p=0.05$; (c) TP, $p=0.1$; (d) $\mathrm{NH}_{3}-\mathrm{N}, p=0.01$; (e) $\mathrm{NH}_{3}-\mathrm{N}, p=0.05$; (f) $\left.\mathrm{NH}_{3}-\mathrm{N}, p=0.1\right]$ 


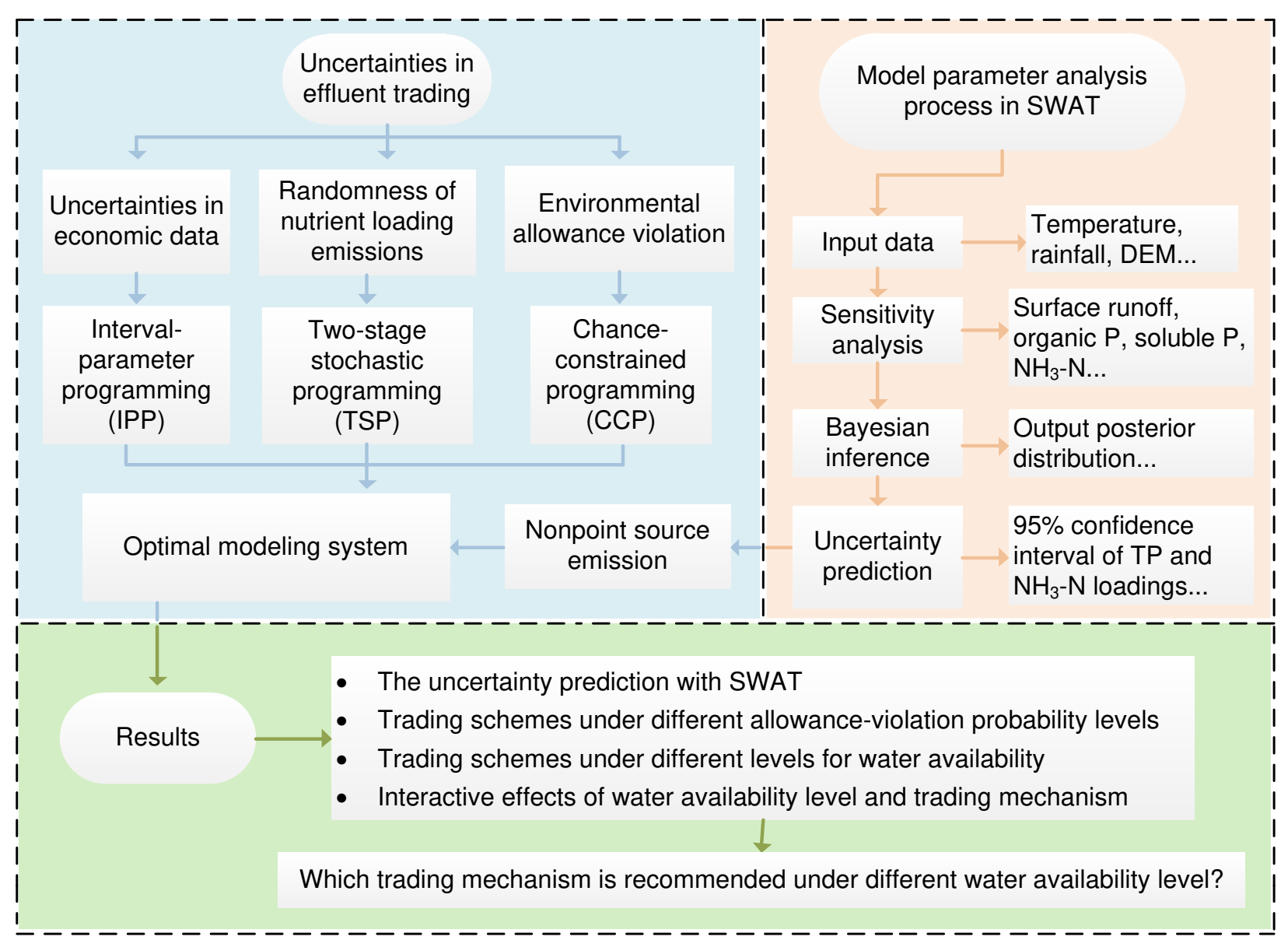

Figure 1 Framework of a Bayesian simulation-based multi-watershed effluent trading designing model (BS-METM) 


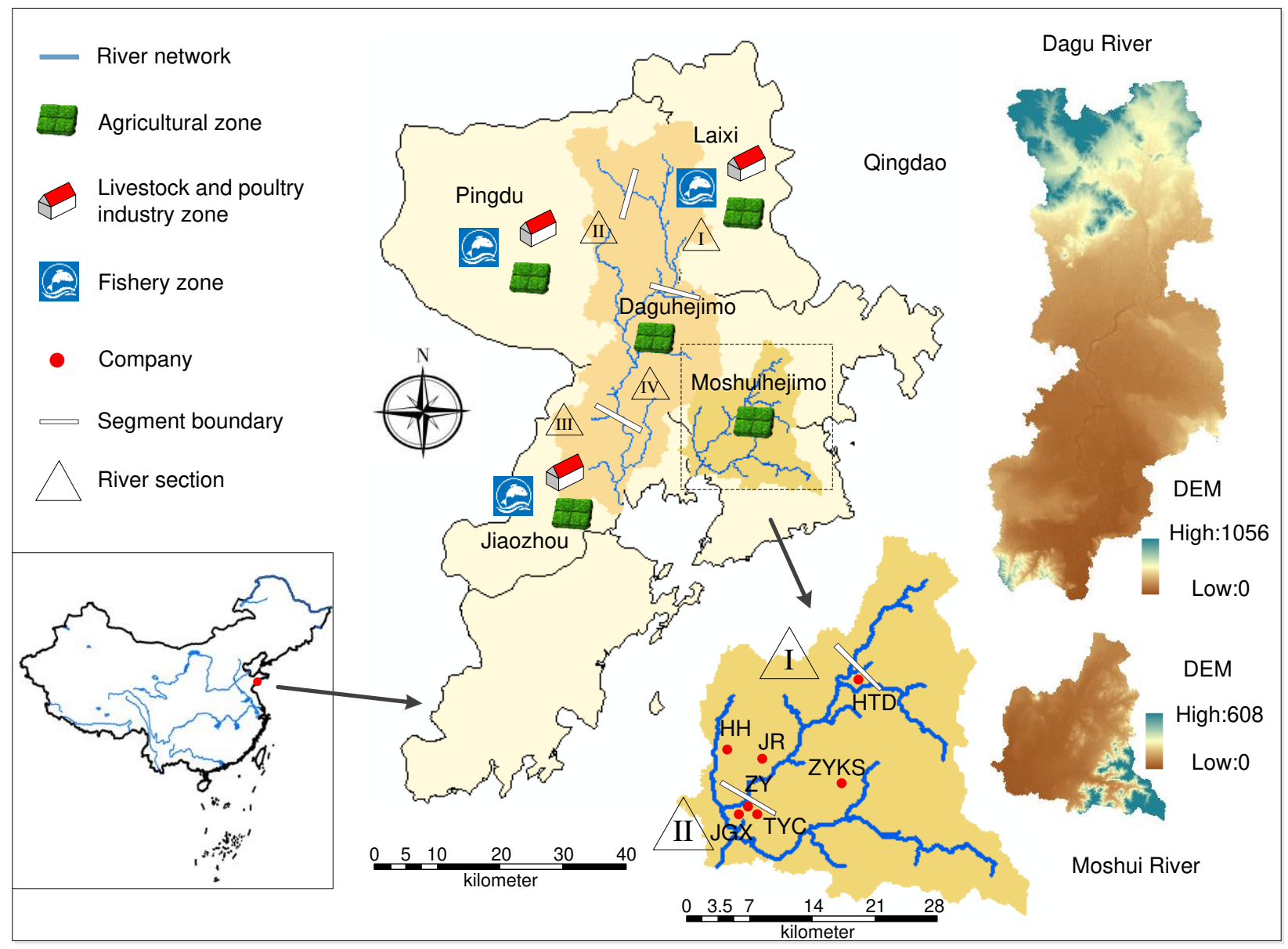

Figure 2 Location and pollution sources of Daguhe and Moshuihe watersheds

Footnote: ZY, TYC, HH, ZYKS, HTD, JR and JGX represent Qingdao Zhengyuan Iron and Steel Co., Ltd, Qingdao Tongyuanchang Steel Co., Ltd, Qingdao Hehe Chemical Co., Ltd, Qingdao Zeyukaisheng Machinery Manufacturing Co., Ltd, Qingdao Huataida Machinery Manufacturing Co., Ltd, Qingdao Jingrui Machinery Manufacturing Co., Ltd and Qingdao Jinguangxin Textile Co., Ltd. Respectively. 


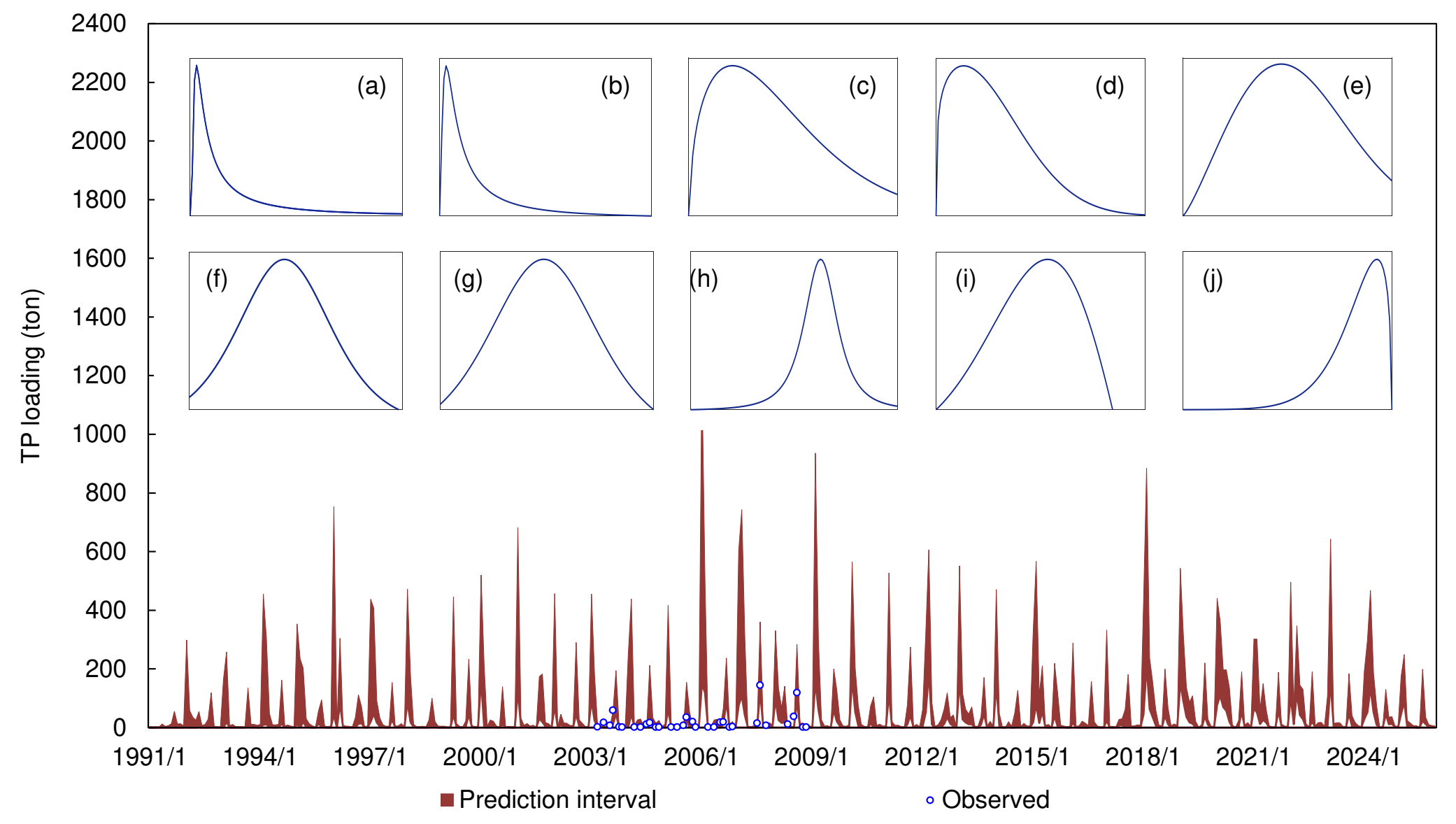

Figure 3 The prediction intervals of TP loading 


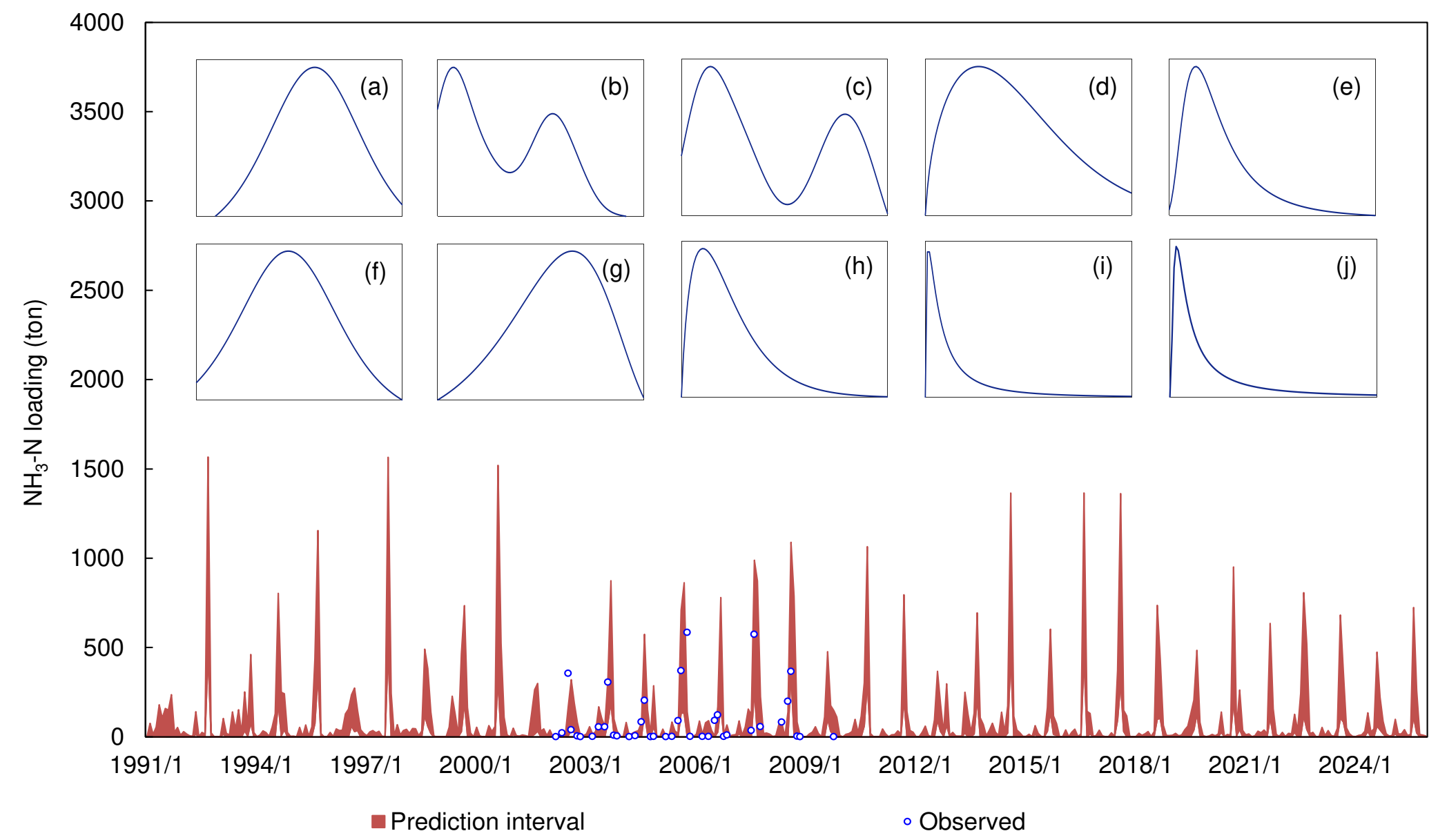

Figure 4 The prediction intervals of $\mathrm{NH}_{3}-\mathrm{N}$ loading 

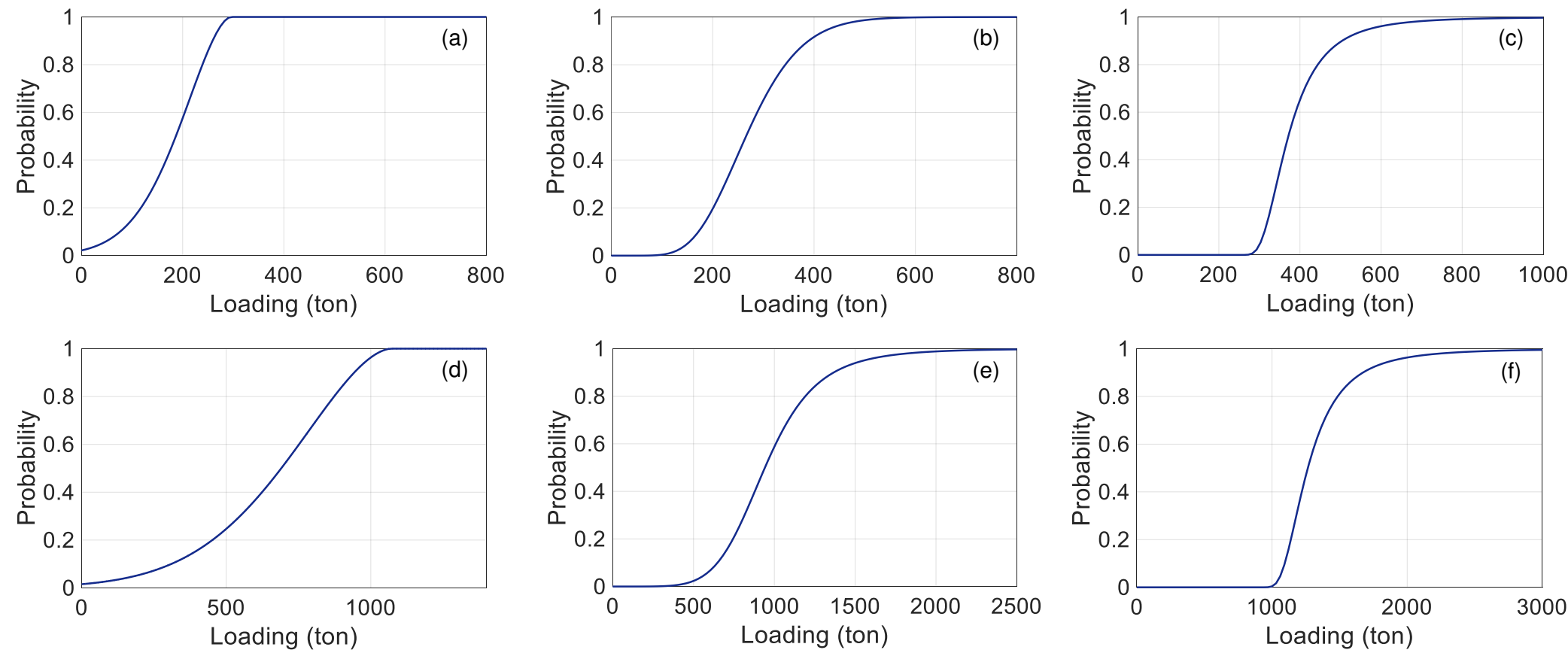

Figure 5 Cumulated distribution functions of TP loadings [(a) low TP loading level for lower bound, (b) medium TP loading level for lower bound, (c) high TP loading level for lower bound, (d) low TP loading level for upper bound, (e) medium TP loading level for upper bound, (f) high TP loading level for upper bound] 

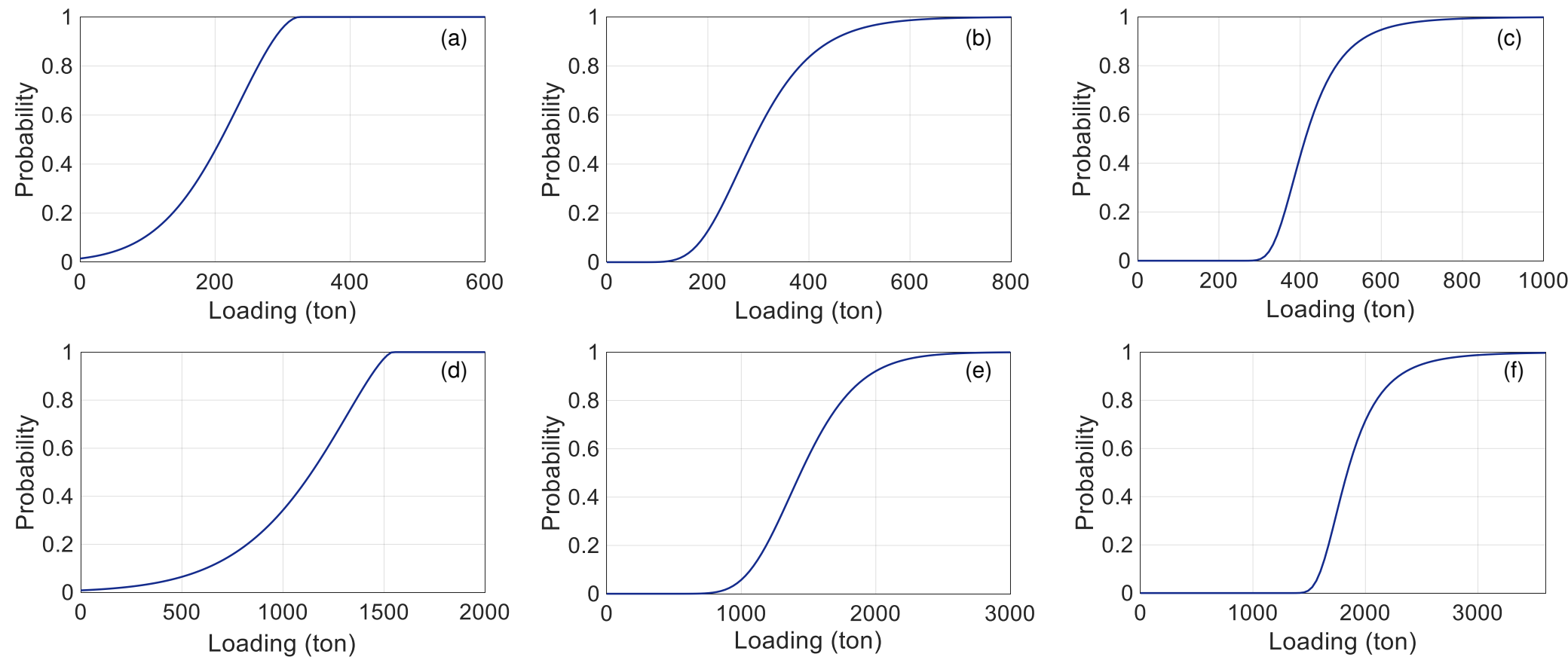

Figure 6 Cumulated distribution functions of $\mathrm{NH}_{3}-\mathrm{N}$ loadings [(a) low $\mathrm{NH}_{3}-\mathrm{N}$ loading level for lower bound, (b) medium $\mathrm{NH}_{3}-\mathrm{N}$ loading level for lower bound, (c) high $\mathrm{NH}_{3}-\mathrm{N}$ loading level for lower bound, (d) low $\mathrm{NH}_{3}-\mathrm{N}$ loading level for upper bound, (e) medium $\mathrm{NH}_{3}-\mathrm{N}$ loading level for upper bound, (f) high $\mathrm{NH}_{3}-\mathrm{N}$ loading level for upper bound] 

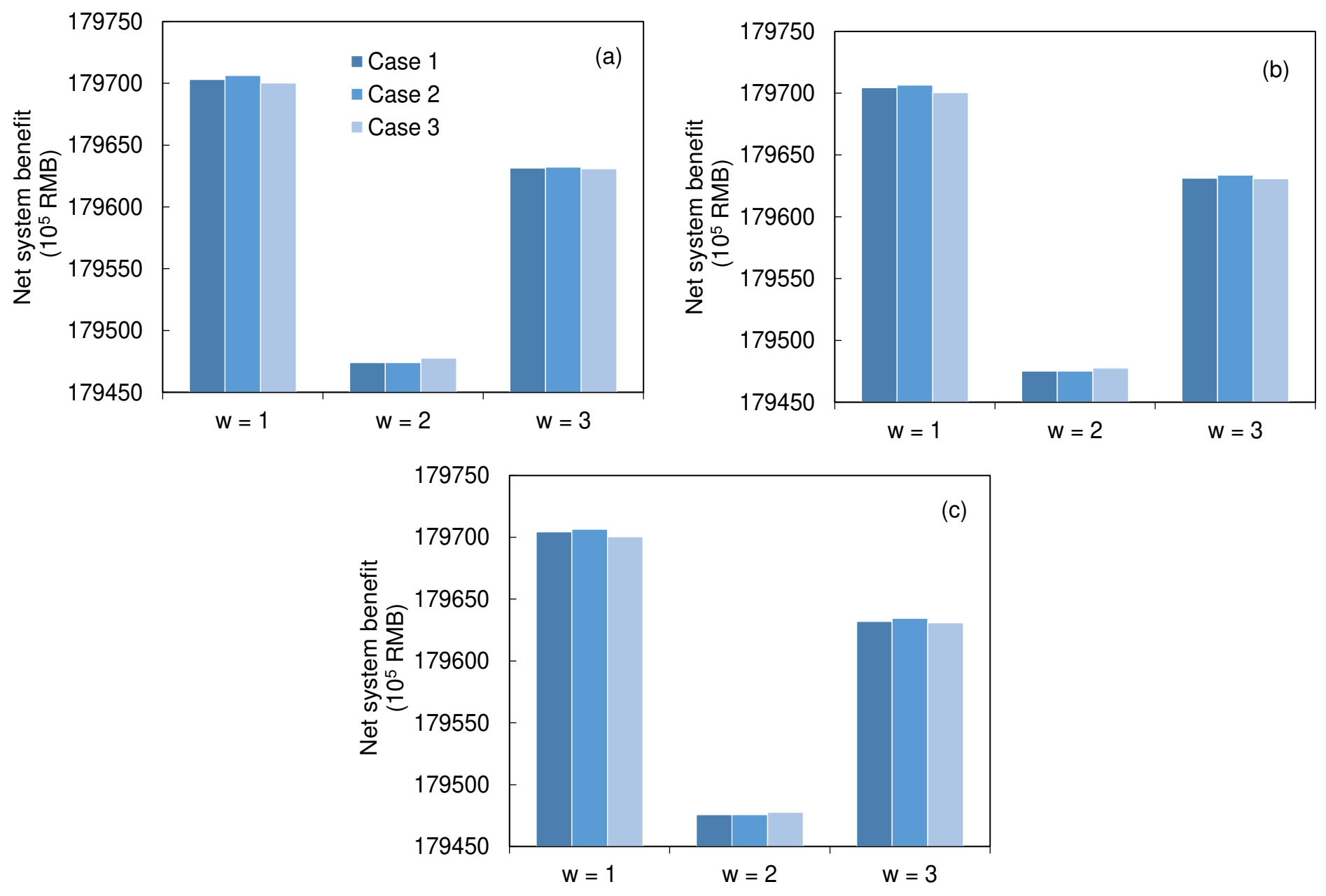

Figure 7 Net system benefits under intra-watershed trading, cross-watershed trading and non-trading cases [(a) $p=0.01$, (b) $p=$ $0.05,(\mathrm{c}) p=0.1]$ 

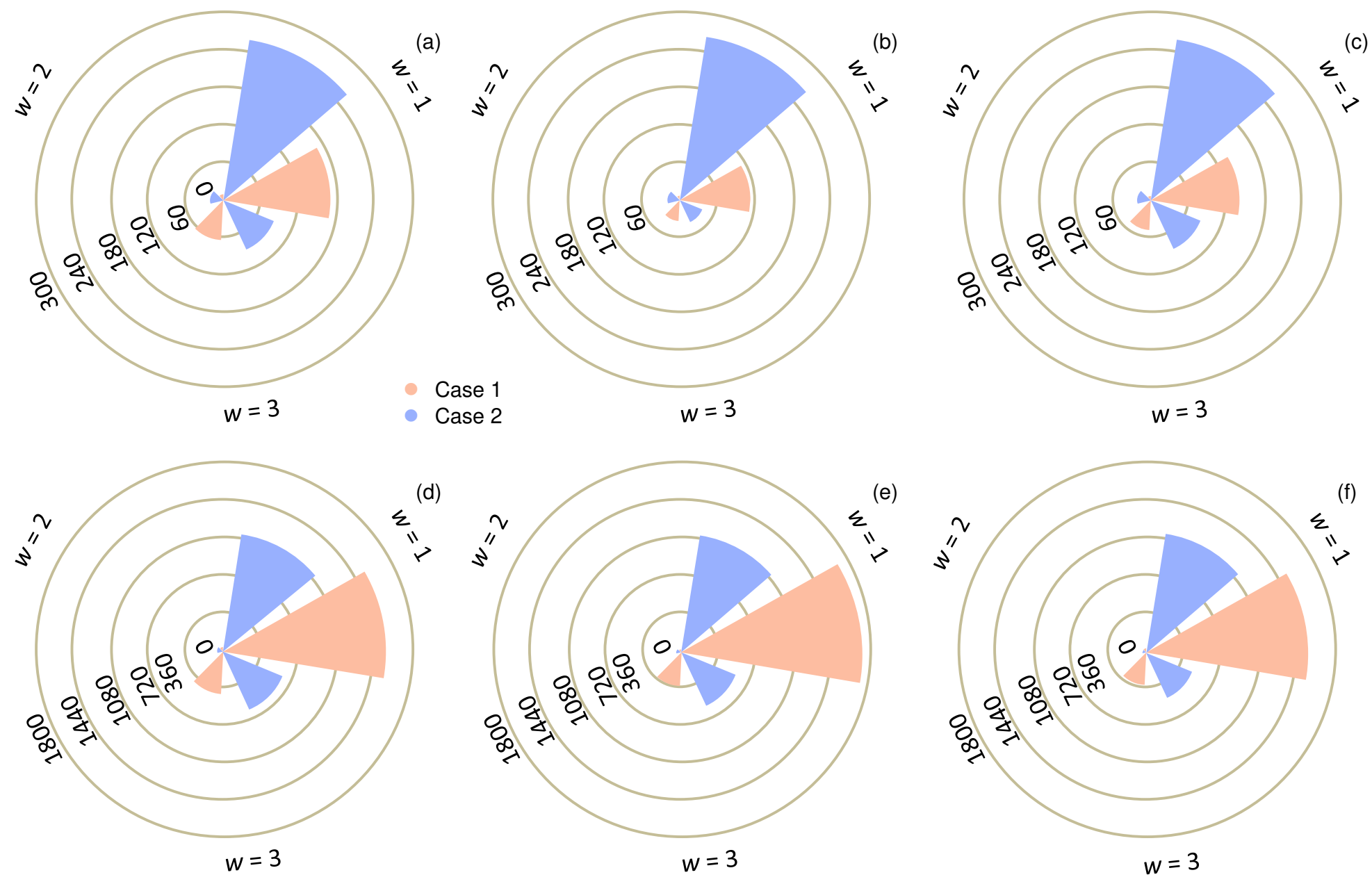

Figure 8 The total trading amounts under Cases 1 and 2 [(a) TP, $p=0.01$; (b) TP, $p=0.05$, (c) TP, $p=0.1$; (d) NH$-\mathrm{N}, p=0.01$;

(e) $\mathrm{NH}_{3}-\mathrm{N}, p=0.05$, (f) $\left.\mathrm{NH}_{3}-\mathrm{N}, p=0.1\right]$ 
(a)
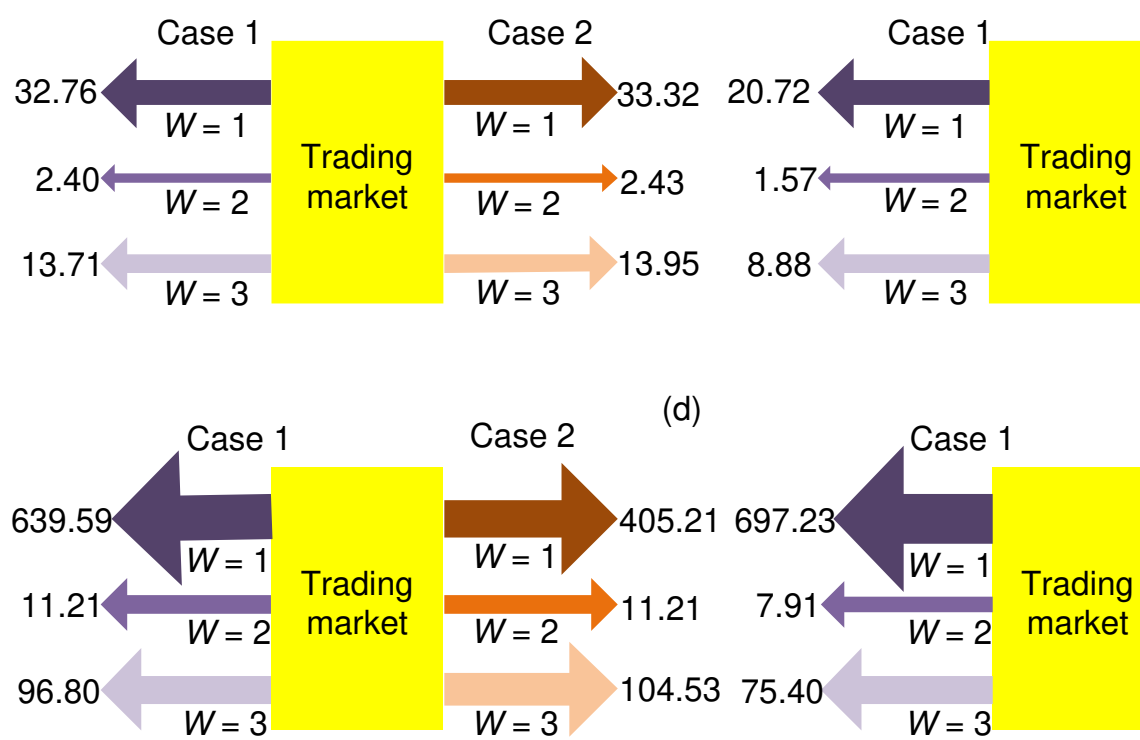

(b)
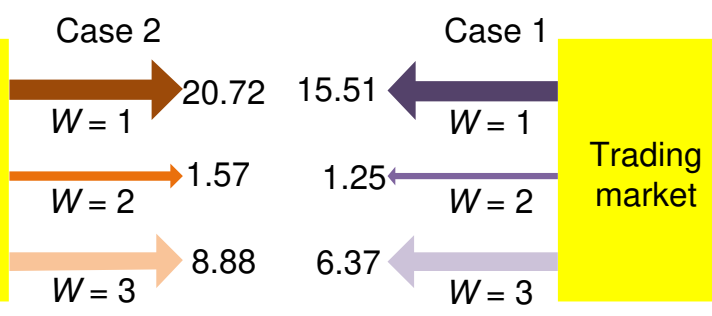

Case 2

(c)
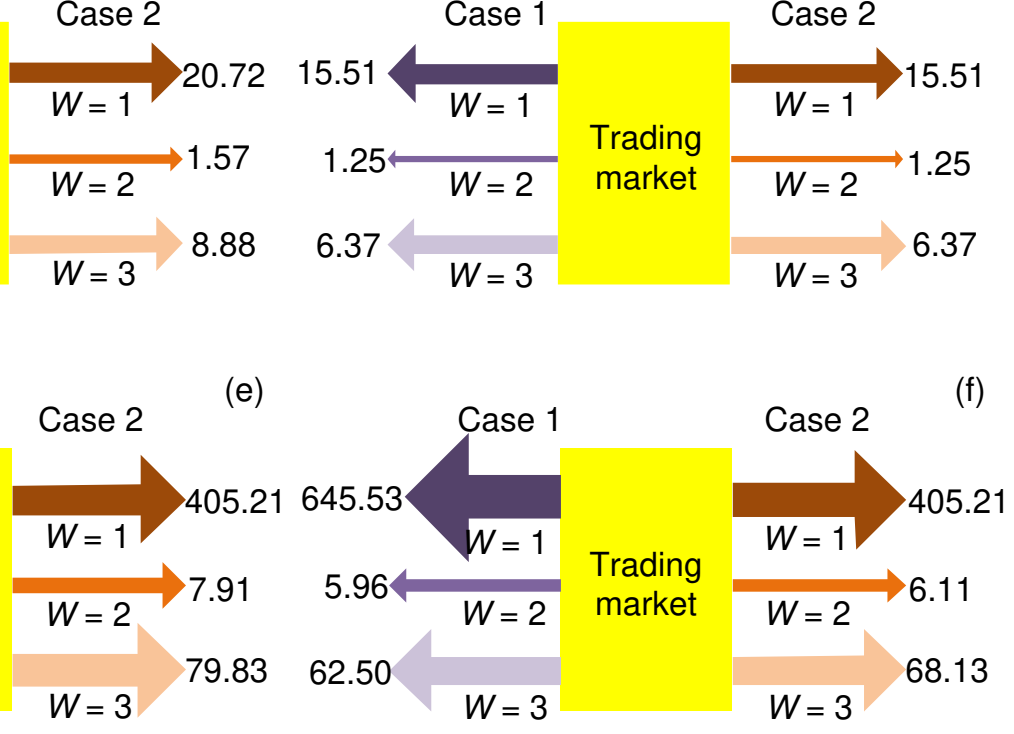

Figure 9 The eliminated trading amounts of tradings under Cases 1 and 2 for TP and $\mathrm{NH}_{3}-\mathrm{N}[(\mathrm{a}) \mathrm{TP}, p=0.01$; (b) TP, $p=0.05$; (c) $\mathrm{TP}, p=0.1 ;$ (d) $\mathrm{NH}_{3}-\mathrm{N}, p=0.01 ;$ (e) $\mathrm{NH}_{3}-\mathrm{N}, p=0.05$; (f) $\left.\mathrm{NH}_{3}-\mathrm{N}, p=0.1\right]$ 

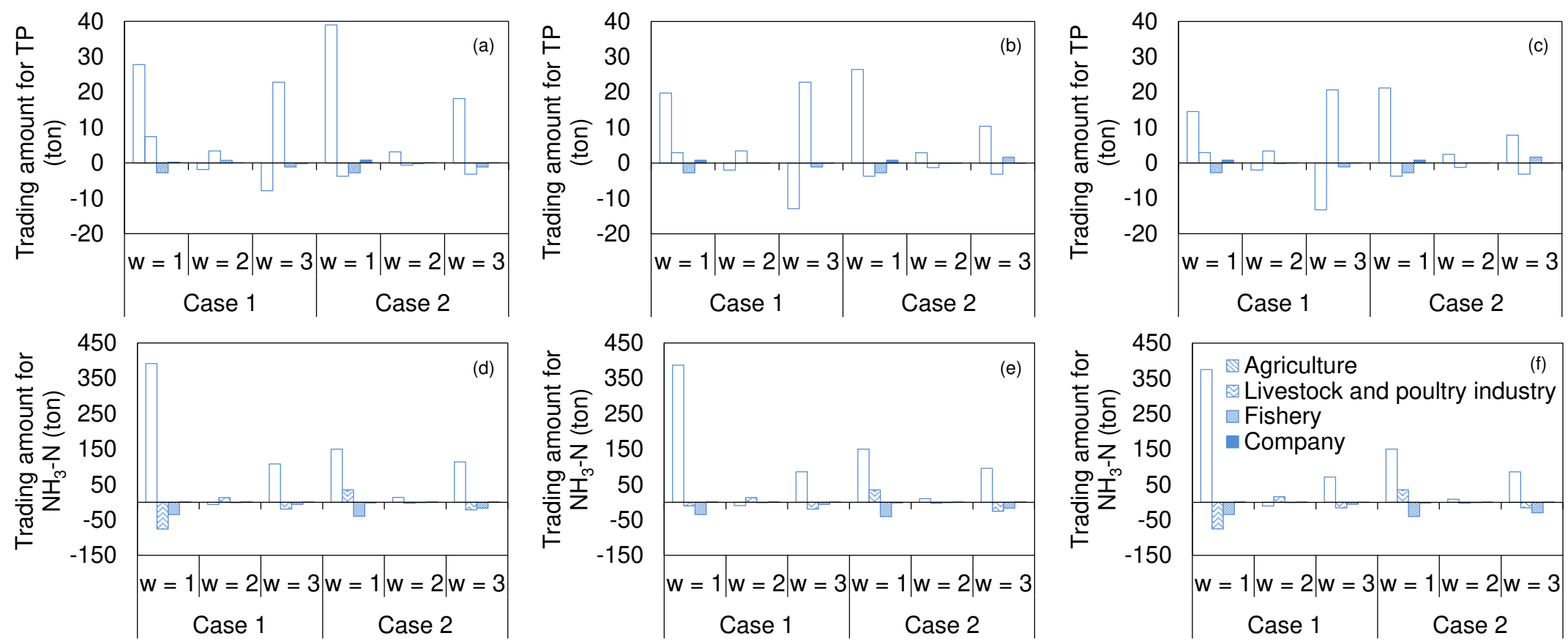

Figure $10 \mathrm{TP}$ and $\mathrm{NH}_{3}-\mathrm{N}$ detailed trading process for agriculture, livestock and poultry industry, fishery and company [(a) TP, $p$ $=0.01 ;$ (b) TP, $p=0.05 ;$ (c) TP, $p=0.1 ;$ (d) $\mathrm{NH}_{3}-\mathrm{N}, p=0.01$; (e) $\mathrm{NH}_{3}-\mathrm{N}, p=0.05$; (f) $\left.\mathrm{NH}_{3}-\mathrm{N}, p=0.1\right]$ 


\section{Conflicts of interest/Competing intrersts}

$\square$ The authors declare that they have no known competing financial interests or personal relationships that could have appeared to influence the work reported in this paper.

$\square$ The authors declare the following financial interests/personal relationships which may be considered as potential competing interests:

The authors declare that they have no known competing financial interests or personal relationships that could
have appeared to influence the work reported in this paper.




\section{Code availability Not applicable}


Figures

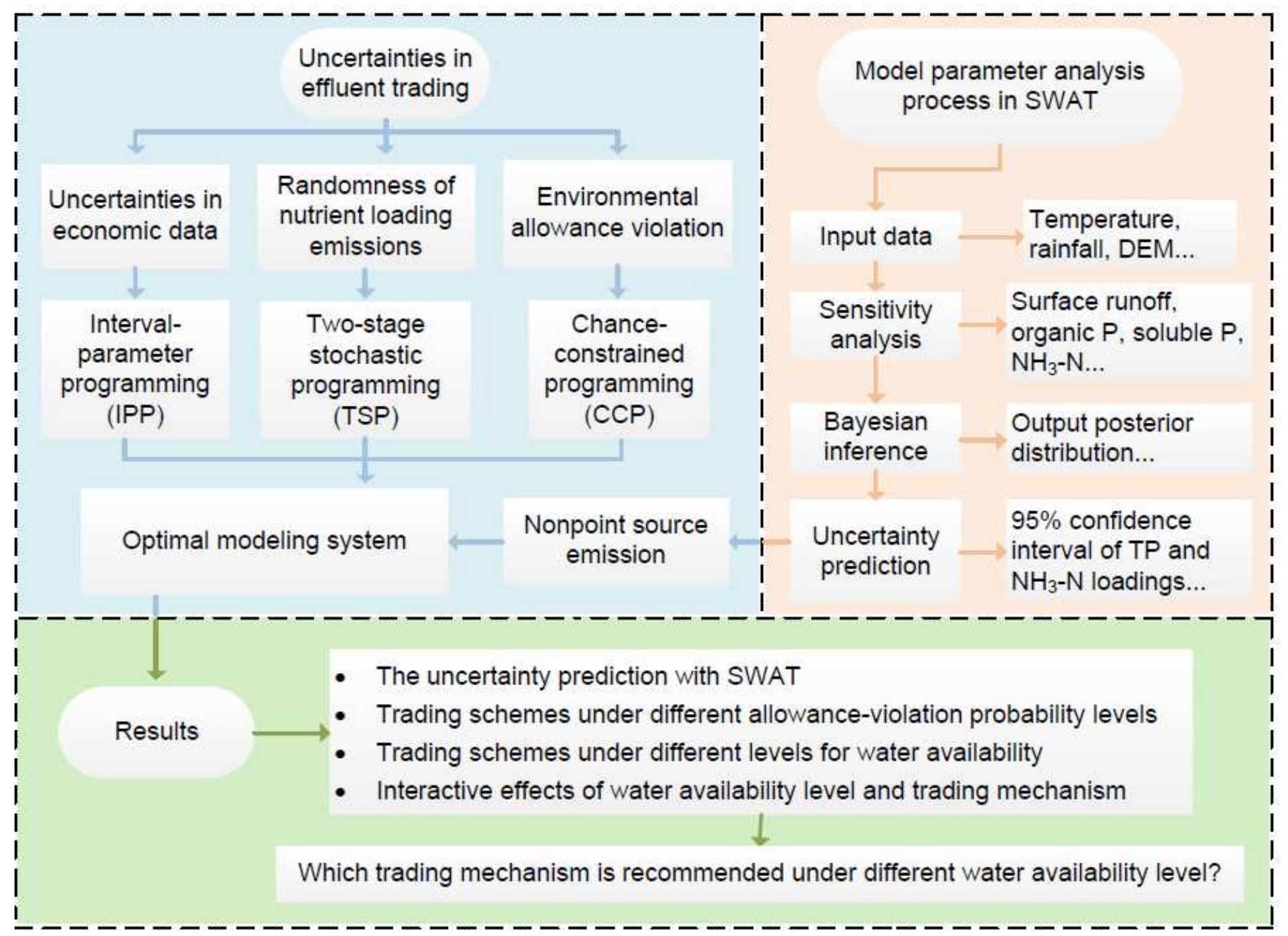

Figure 1

Framework of a Bayesian simulation-based multi-watershed effluent trading designing model (BS-METM) 


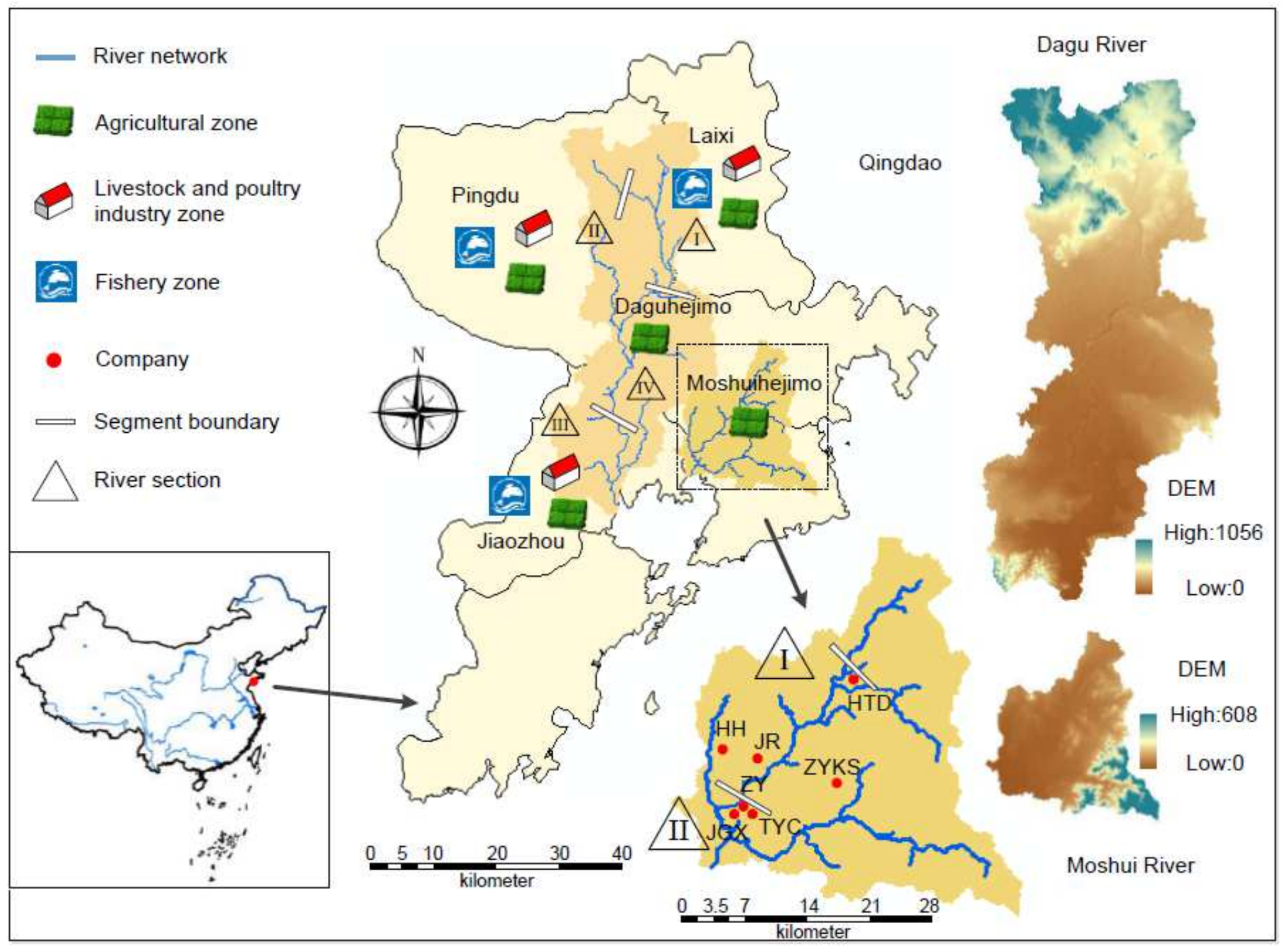

Figure 2

Location and pollution sources of Daguhe and Moshuihe watersheds Footnote: ZY, TYC, HH, ZYKS, HTD, JR and JGX represent Qingdao Zhengyuan Iron and Steel Co., Ltd, Qingdao Tongyuanchang Steel Co., Ltd, Qingdao Hehe Chemical Co., Ltd, Qingdao Zeyukaisheng Machinery Manufacturing Co., Ltd, Qingdao Huataida Machinery Manufacturing Co., Ltd, Qingdao Jingrui Machinery Manufacturing Co., Ltd and Qingdao Jinguangxin Textile Co., Ltd. Respectively. Note: The designations employed and the presentation of the material on this map do not imply the expression of any opinion whatsoever on the part of Research Square concerning the legal status of any country, territory, city or area or of its authorities, or concerning the delimitation of its frontiers or boundaries. This map has been provided by the authors. 


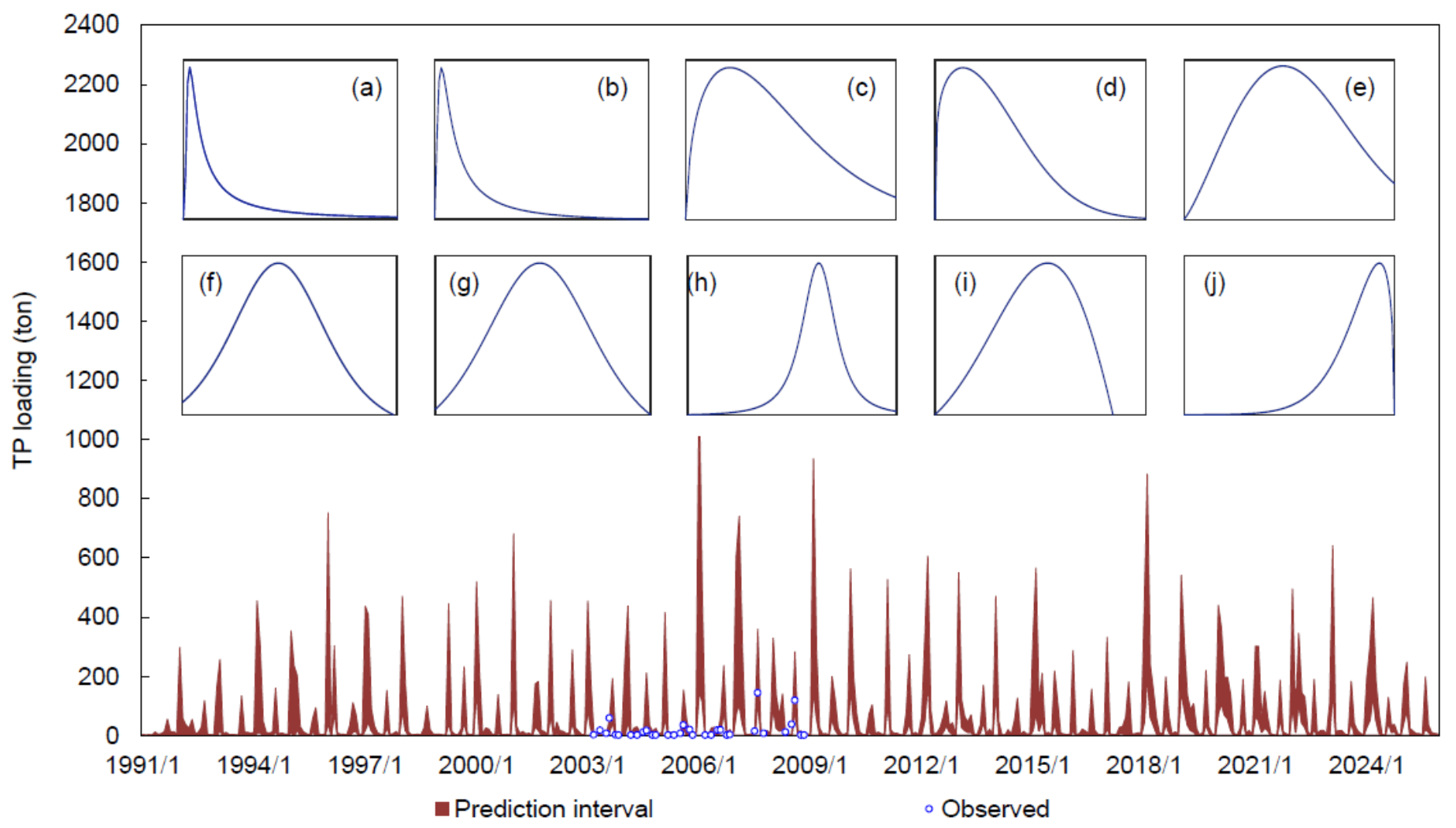

Figure 3

The prediction intervals of TP loading 


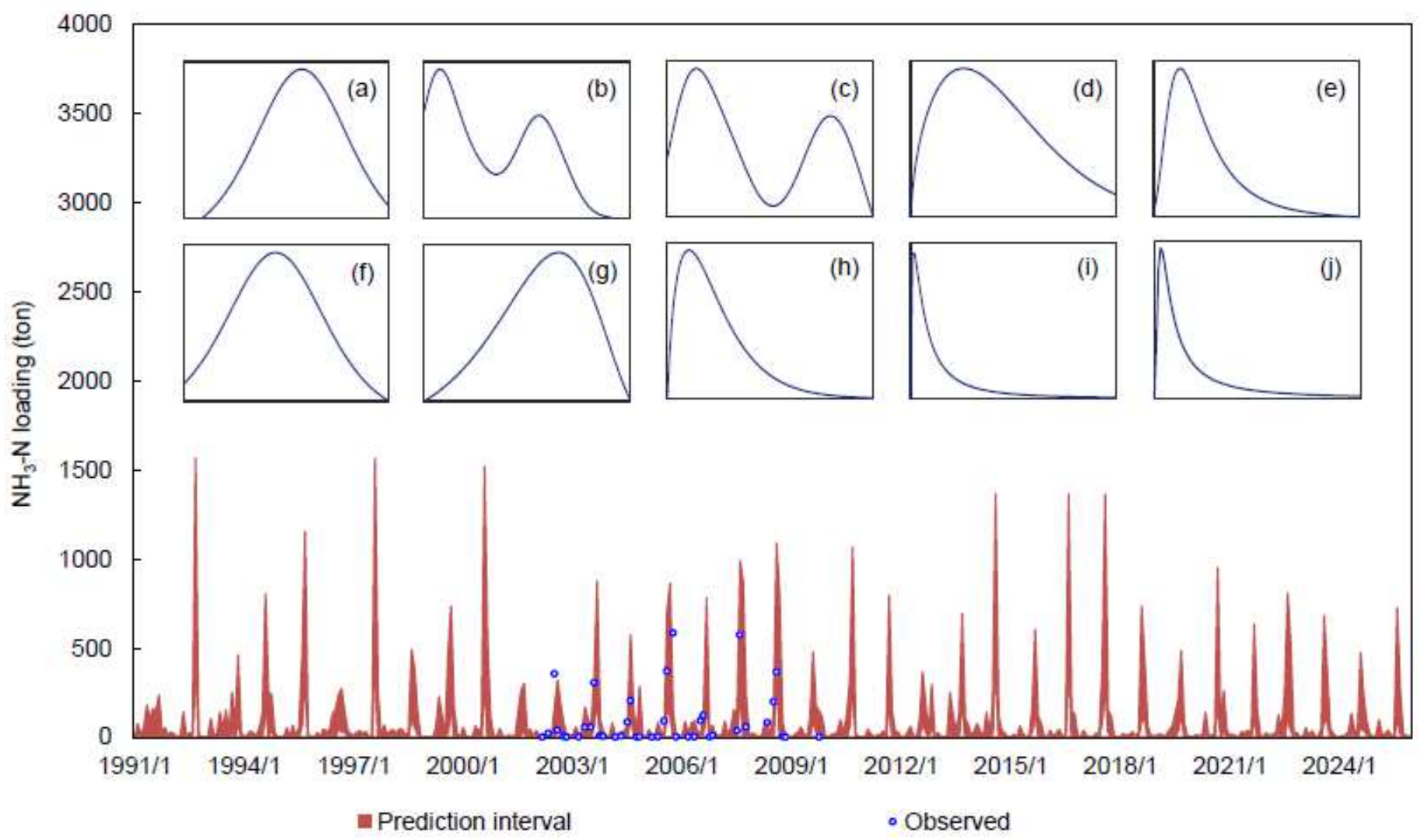

Figure 4

The prediction intervals of NH3-N loading
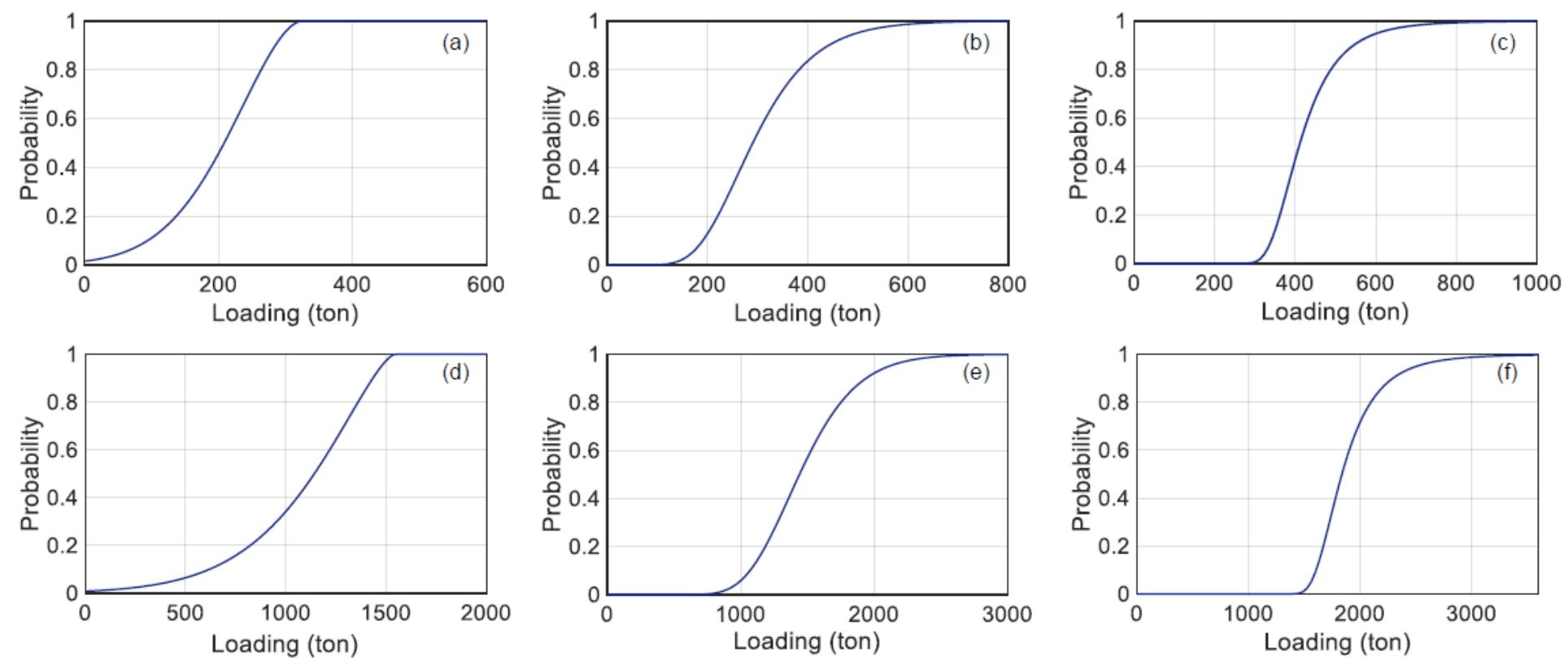

Figure 6 
Cumulated distribution functions of NH3-N loadings [(a) low NH3-N loading level for lower bound, (b) medium NH3-N loading level for lower bound, (c) high NH3-N loading level for lower bound, (d) low NH3-N loading level for upper bound, (e) medium NH3-N loading level for upper bound, (f) high NH3-N loading level for upper bound]

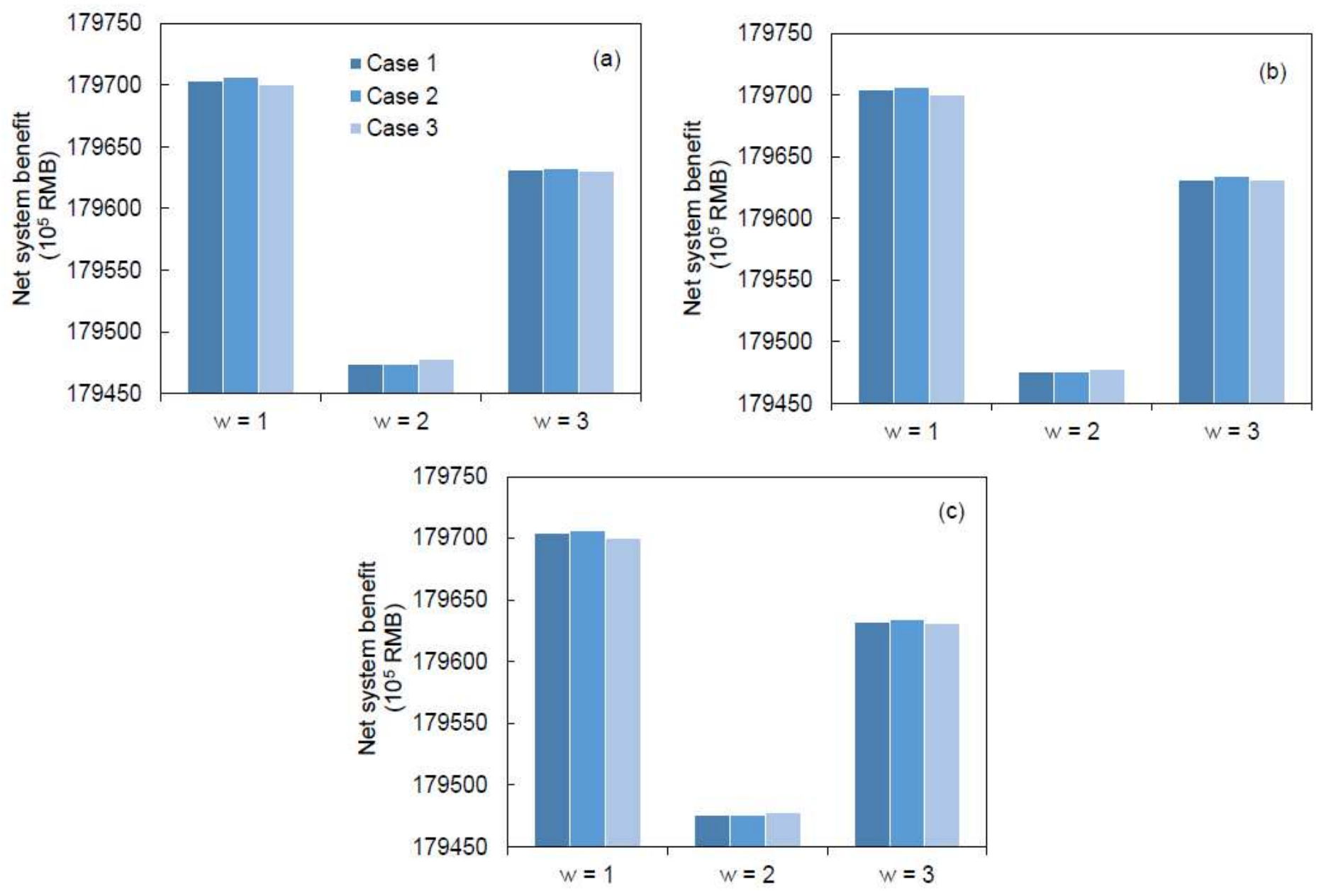

\section{Figure 7}

Net system benefits under intra-watershed trading, cross-watershed trading and non-trading cases $[(a) p=$ 0.01 , (b) $p=0.05$, (c) $p=0.1]$ 

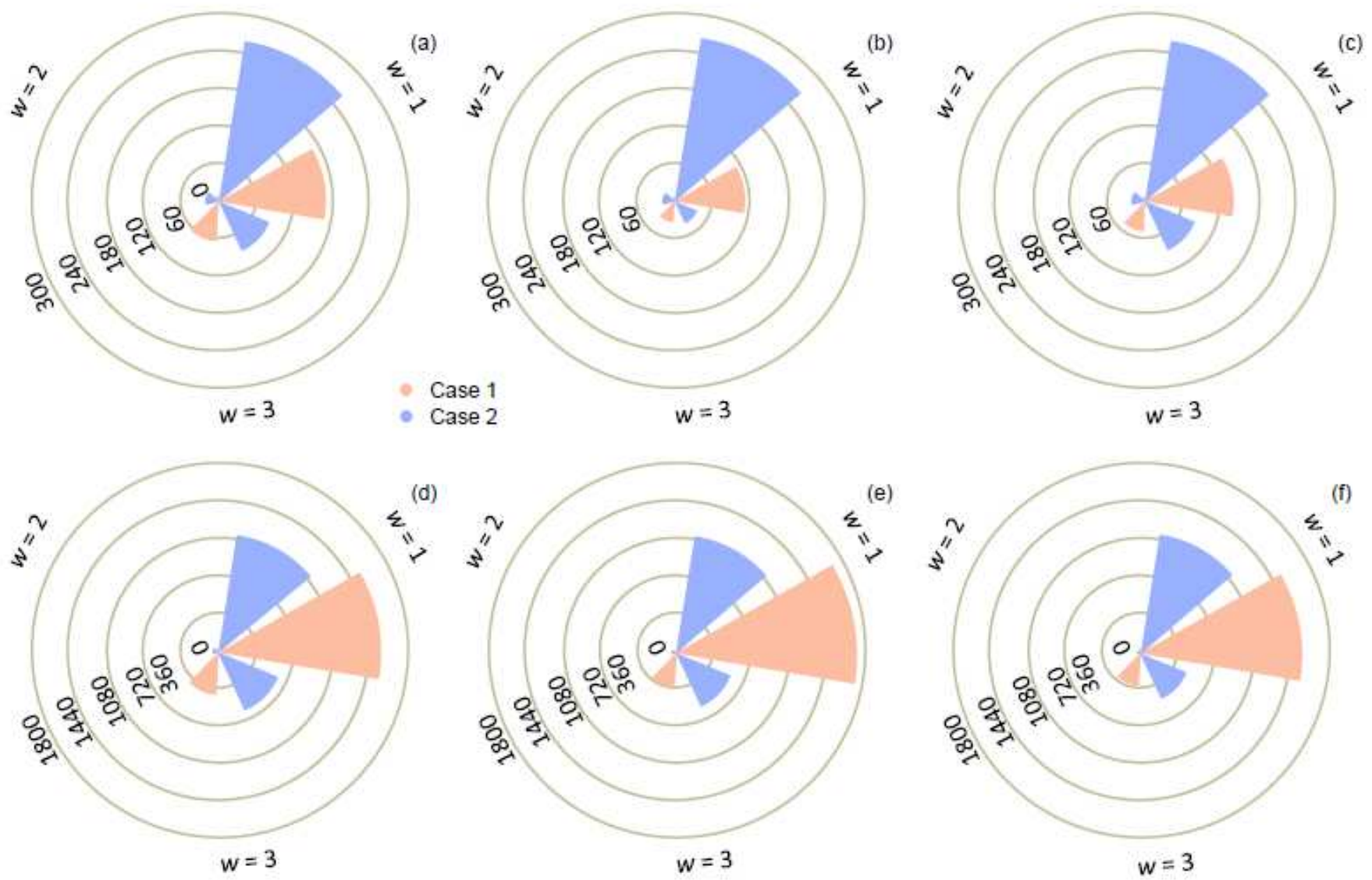

\section{Figure 8}

The total trading amounts under Cases 1 and 2 [(a) TP, $p=0.01$; (b) TP, $p=0.05$, (c) TP, $p=0.1$; (d) NH3-N, $p=0.01 ;(e) N H 3-N, p=0.05$, (f) NH3-N, $p=0.1]$

(a)
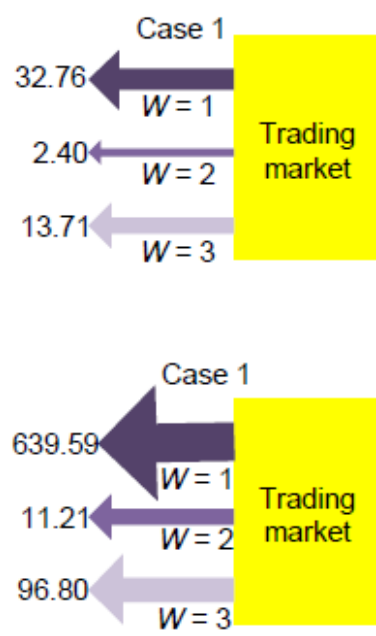

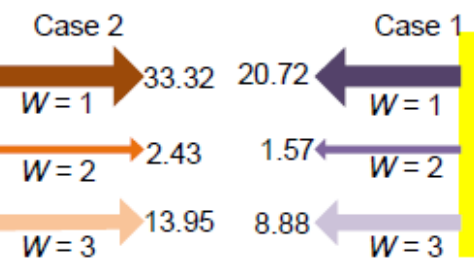

(d)

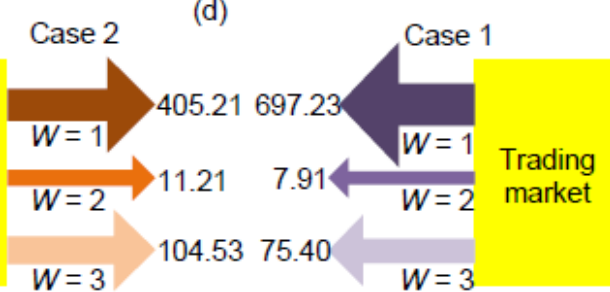

(b)
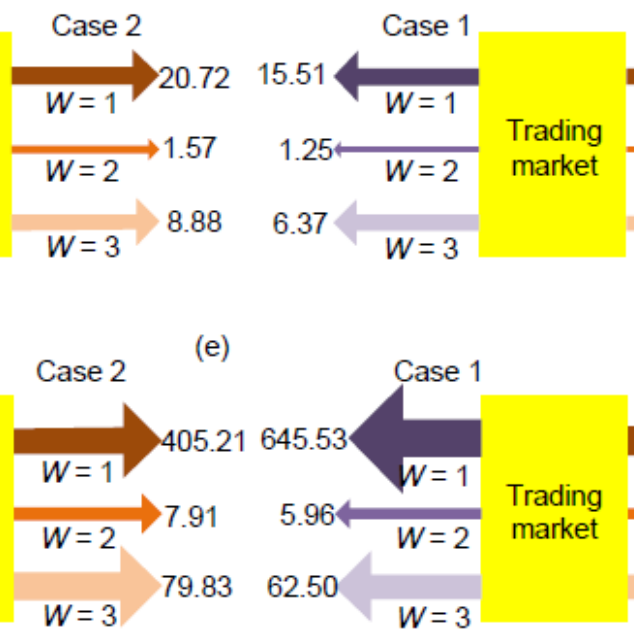

Case 2

(c)

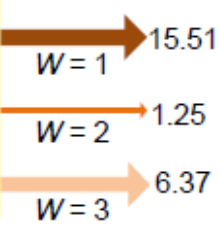

Case 2

(f)

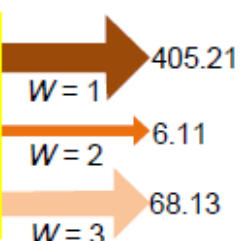

Figure 9 
The eliminated trading amounts of tradings under Cases 1 and 2 for TP and NH3-N [(a) TP, $p=0.01$; (b) TP, $p=0.05 ;$ (c) TP, $p=0.1 ;$ (d) NH3-N, $p=0.01$; (e) NH3-N, $p=0.05$; (f) NH3-N, $p=0.1$ ]
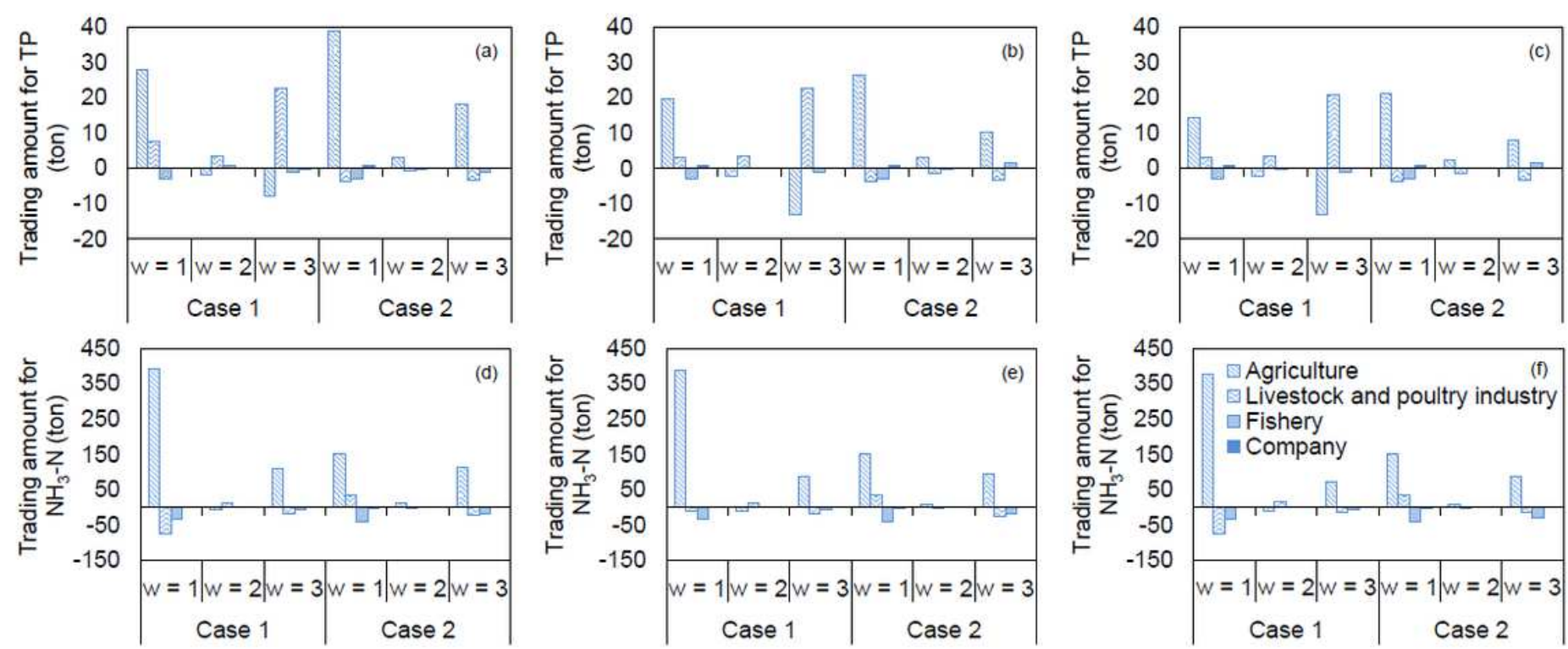

Figure 10

TP and NH3-N detailed trading process for agriculture, livestock and poultry industry, fishery and company [(a) TP, $p=0.01$; (b) TP, $p=0.05$; (c) TP, $p=0.1$; (d) NH3-N, p = 0.01; (e) NH3-N, p = 0.05; (f) NH3$N, p=0.1]$

\section{Supplementary Files}

This is a list of supplementary files associated with this preprint. Click to download.

- GraphicalAbstract.png 